\title{
HANDLEBODY CONSTRUCTION OF STEIN SURFACES
}

\author{
Robert E. GompF*
}

December 3, 1996

\begin{abstract}
The topology of Stein surfaces and contact 3-manifolds is studied by means of handle decompositions. A simple characterization of homeomorphism types of Stein surfaces is obtained - they correspond to open handlebodies with all handles of index $\leq 2$. An uncountable collection of exotic $\mathbb{R}^{4}$ 's is shown to admit Stein structures. New invariants of contact 3-manifolds are produced, including a complete (and computable) set of invariants for determining the homotopy class of a 2-plane field on a 3-manifold. These invariants are applicable to Seiberg-Witten theory. Several families of oriented 3-manifolds are examined, namely the Seifert fibered spaces and all surgeries on various links in $S^{3}$, and in each case it is seen that "most" members of the family are the oriented boundaries of Stein surfaces.
\end{abstract}

\section{Introduction.}

It is becoming evident that low-dimensional topology is intimately connected with the topology of complex, symplectic and contact manifolds. The differential topology of closed 4-manifolds is entwined with that of complex surfaces and symplectic 4-manifolds, as is clear from Donaldson theory (for example [Do]) and more recent developments involving the Seiberg-Witten equations and Gromov invariants (for example, Taubes $[\mathrm{T}]$ ). Cutting and pasting leads us to consider manifolds with boundary, whose corresponding geometric analogs are compact complex and symplectic manifolds with boundaries that inherit compatible tight contact structures. The topology of 3-manifolds with tight contact structures is subtle and still mysterious, as is the question of when they bound complex or symplectic 4-manifolds. There are some similarities, however, with the study of taut foliations on 3-manifolds, which has had a profound influence on 3-manifold topology. Considering the interiors of complex or symplectic manifolds with contact boundaries, we are led to the notion of a Stein manifold, which is perhaps the most natural notion of an open complex or exact symplectic manifold that is "nice at infinity." It is natural to ask which open 4-manifolds admit Stein structures. Eliashberg [E2] proved that Stein manifolds can be characterized in terms of handle decompositions. In the present article, we will use Eliashberg's theorem to study Stein surfaces (real dimension 4), producing a simple characterization of their homeomorphism types and some new examples such as Stein structures on exotic $\mathbb{R}^{4}$ 's. We will also use handlebodies to study contact 3-manifolds and their holomorphic fillings, i.e., compact complex surfaces with contact boundaries.

We begin by discussing Stein manifolds. (See [E2], [E6] for more details and Section 1 for some key definitions in low dimensions.) Stein manifolds are complex manifolds (necessarily noncompact) that admit proper holomorphic embeddings in $\mathbb{C}^{N}$ for sufficiently large $N$. They carry exact Kähler structures, and an equivalent notion can be formulated in terms of symplectic structures [EG]. A complex manifold $X$ is Stein if and only if it admits an "exhausting strictly plurisubharmonic function," which is essentially characterized as being a proper function $f: X \rightarrow \mathbb{R}$ that is bounded below and can be assumed a Morse function, whose level sets $f^{-1}(c)$ are "strictly pseudoconvex" (away from critical points), where $f^{-1}(c)$ is oriented as the boundary of the complex manifold $f^{-1}(-\infty, c]$. Strict pseudoconvexity implies, and for $X$ of real dimension 4 is equivalent to asserting, that $f^{-1}(c)$ inherits a contact structure determining its given orientation.

\footnotetext{
* Partially supported by NSF grant DMS 9301524.
} 
We will call a compact, complex $X$ with boundary a Stein domain if it admits a strictly plurisubharmonic function such that the boundary $\partial X$ is a level set. Then $\partial X$ will be strictly pseudoconvex and int $X$ will be a Stein manifold (with the same level set structure as $X$ ). Conversely, if $f: X \rightarrow \mathbb{R}$ is an exhausting strictly plurisubharmonic function on a Stein manifold, then $f^{-1}(-\infty, c]$ will be a Stein domain for any regular value $c$. Thus, we can almost interchangeably talk about Stein domains and Stein manifolds in the presence of (exhausting) strictly plurisubharmonic Morse functions with only finitely many critical points. We will call a Stein manifold or domain of complex dimension 2 a Stein surface (with boundary). Any compact, complex surface with nonempty, strictly pseudoconvex boundary can be made Stein in this sense by deforming it and blowing down any exceptional curves [Bo].

Eliashberg's theorem [E2] characterizing Stein manifolds of complex dimension $n>2$ can be stated as follows. (For an independent treatment of handle addition in the symplectic category, see $[\mathrm{W}]$. .)

Theorem 0.1 (Eliashberg). For $n>2$, a smooth, almost-complex, open 2n-manifold admits a Stein structure if and only if it is the interior of a (possibly infinite) handlebody without handles of index $>n$. The Stein structure can be chosen to be homotopic to the given almost-complex structure, and the given handle decomposition is induced by an exhausting strictly plurisubharmonic Morse function. Similarly, a smooth, almost complex, compact $2 n$-manifold $(n>2)$ with a handle decomposition without handles of index $>n$ admits a homotopic Stein domain structure with a suitable plurisubharmonic function inducing the handle decomposition.

Thus, in high dimensions, an almost-complex structure and a handle decomposition with no handles of index above the middle dimension are sufficient to guarantee the existence of a Stein structure. Implicit in the same paper is a theorem in the $n=2$ case (which is also due to Eliashberg, cf. [E5], but not explicitly published). We state it as Theorem 1.3 below. When $n=2$, the required almost-complex structure always exists (for oriented handlebodies as in the theorem). The main difference between this case and the $n>2$ case, however, is that when $n=2$, there is a serious restriction on the allowable framings by which the 2-handles are attached. In practice, it is a delicate matter to determine whether a given 4-manifold admits a handle structure with allowable framings, and if so, which almost-complex structures can be so realized. Thus, while Eliashberg has characterized Stein manifolds in all dimensions in terms of differential topology, the case of 4-manifolds and Stein surfaces still presents great challenges in applications. It is these applications that the present article addresses.

One application is the characterization of Stein surfaces (without boundary) up to homeomorphism (Section 3). Freedman's work on topological 4-manifolds [F], [FQ] shows that if we forget about smooth structures, then the theory of 4-manifolds (with small $\pi_{1}$ ) becomes similar to that of higher dimensional manifolds, hence, easier to understand. In that spirit, we prove (Theorem 3.1) that Eliashberg's theorem in high dimensions applies up to homeomorphism to 4-manifolds. That is, an open, oriented topological 4-manifold $X$ is (orientation-preserving) homeomorphic to a Stein surface if and only if it is the interior of a topological (or smooth) handlebody without handles of index $>2$, and if so, then any almost-complex structure can be so realized. The given handle structure will not necessarily come from a plurisubharmonic (or even smooth Morse) function on the Stein surface, however. As is typical of examples obtained from Freedman theory, these Stein surfaces will tend to have smooth structures that are in some sense "exotic." We cannot expect them to admit proper Morse functions with finitely many critical points. As an example (Theorem 3.3), $\mathbb{C} P^{2}$ minus a point admits an uncountable family of diffeomorphism types of Stein exotic smooth structures, none of which admit proper Morse functions with finitely many critical points, and none of which contain a smoothly embedded sphere representing a generator of the homology. Similarly, any $\mathbb{R}^{2}$-bundle over $S^{2}$ admits a Stein exotic smooth structure containing no generating smoothly embedded spheres. In contrast, the standard smooth structure on $\mathbb{C} P^{2}-\operatorname{int} B^{4}$ (or on $S^{2} \times D^{2}$ ) cannot be realized by a compact Stein surface (or even a convex symplectic manifold), by uniqueness of fillings [Gro], [E3]. As a further application, we show that $\mathbb{R}^{4}$ admits uncountably many exotic smooth structures that can be realized as Stein surfaces (Theorem 3.4). None of these admit proper Morse functions with finitely many critical points, but subject to that constraint, one example has a handle decomposition that is remarkably simple. (It is the example of a simple exotic $\mathbb{R}^{4}$ constructed by Bižaca and the author in [BG].) 
One of the main techniques of this paper is to describe handle decompositions of Stein surfaces explicitly using Kirby calculus. While this method has already been applied in simple cases without 1-handles [E5], the general case is more delicate. In Section 2, we establish a standard form for any handle decomposition obtained from a strictly plurisubharmonic function on a compact Stein surface. We do this via a standard form for Legendrian links in the connected sum $\# n S^{1} \times S^{2}$ that allows us to define and compute the rotation number and Thurston-Bennequin invariant of each link component (even those that are nontrivial in $H_{1}$ ). We also provide a complete reduction from Legendrian link theory in $\# n S^{1} \times S^{2}$ to a theory of diagrams by introducing a complete set of "Reidemeister moves." These diagrams allow us to construct Stein surfaces by drawing pictures. For example, we obtain the above exotic $\mathbb{R}^{4}$ 's in this manner. A Legendrian link diagram in $\# n S^{1} \times S^{2}$ also determines a (positively oriented) contact 3-manifold $(M, \xi)$, namely the oriented boundary of the corresponding compact Stein surface. We say that $(M, \xi)$ is obtained by contact surgery on the Legendrian link, and that $(M, \xi)$ is holomorphically fillable. In Section 5, we construct several families of examples. We realize "most" oriented Seifert fibered 3-manifolds by contact surgery, including all with (possibly nonorientable) base $\neq S^{2}$, and both orientations on many Brieskorn homology spheres (Theorem 5.4 and Corollary 5.5). We show that any Seifert fibered space can be realized in this manner after possibly reversing orientation. For hyperbolic examples, we realize "most" rational surgeries on the Borromean rings (Theorem 5.9).

We also introduce new invariants for distinguishing contact structures. We define a complete set of invariants for determining the homotopy class of an oriented 2-plane field on an oriented 3-manifold $M$. These invariants are readily computable for the boundary of a compact Stein surface presented in standard form. An explicit formula for the 2-dimensional obstruction (which measures the associated spin $^{c}$-structure) is given by Theorem 4.12. The 3-dimensional obstruction (which, by recent work of Kronheimer and Mrowka [KM], distinguishes the grading in Seiberg-Witten-Floer Theory) is given by Definitions 4.2 and 4.15. The construction of these invariants is surprisingly delicate. While a choice of trivialization on the tangent bundle $T M$ reduces the problem to the homotopy classification of maps $M \rightarrow S^{2}$ (which was solved by Pontrjagin around $1940[\mathrm{P}]$ ), the resulting obstructions depend on the choice of trivialization, making them hard to work with directly. Our invariants depend (in the worst case) only on a spin structure and a framing on a certain 1-cycle in $M$, data that one can easily follow through Kirby calculus computations. As an application, we show (Corollary 4.6) that the rotation number (up to sign) and Thurston-Bennequin invariant of a Legendrian knot in $S^{3}$ are both invariants of the contact 3-manifold $M$ obtained by contact surgery on the knot. In particular, the sign of the surgery coefficient is determined by the contact structure on $M$. We then observe that we can easily construct families of different (nonhomotopic and noncontactomorphic) holomorphically fillable contact structures, on a fixed 3-manifold, that cannot be distinguished by the Chern (= Euler) class of $\xi$. This provides new counterexamples to Conjecture 10.3 of [E3]. (Such examples were already known in the weaker case of symplectically fillable structures [Gi2], although these were all homotopic.) We give several corollaries about homotopy classes of 2-plane fields (or equivalently, nowhere zero vector fields or "combings") on 3 -manifolds. We also show (Corollary 4.19) that any contact structure respecting the unusual orientation on the Poincaré homology sphere must have an overtwisted universal cover. Although Conjecture 5.6 asserts that this oriented manifold should admit no fillable structures, we show in Proposition 5.1 that it is common (among lens spaces, for example) for holomorphically fillable contact structures to have finite covers that are overtwisted. In fact, we exploit this phenomenon in Example 5.2 to obtain a more subtle way of distinguishing tight contact structures. We exhibit a 3-manifold with a pair of holomorphically fillable contact structures that are homotopic as plane fields, and distinguish these by whether the corresponding contact structures on a certain 2-fold covering space are tight or overtwisted. Recent advances in gauge theory such as $[\mathrm{KM}]$ are leading to other methods for distinguishing homotopic contact structures; see $[\mathrm{AM}]$ and $[\mathrm{LM}]$.

The author wishes to thank Yasha Eliashberg for many indispensable conversations, and to acknowledge the Isaac Newton Institute for Mathematical Sciences in Cambridge, England for their support during their 1994 program on symplectic and contact topology, at whose lectures by Eliashberg the author was first properly introduced to contact topology and Stein surfaces.

\section{Legendrian links.}


In this section, we review some standard theory of contact 3-manifolds, Legendrian links and their relation with Stein surfaces. Throughout the paper, we will be working with $C^{\infty}$ oriented 2-plane fields $\xi$ on oriented (usually closed) 3-manifolds $M$. Such a 2-plane field can be written as the kernel of a nowhere zero 1-form $\alpha$ on $M$ that is unique up to multiplication by nonzero scalar functions. It is easily verified that the integrability of $\xi$ is equivalent to the condition that $\alpha \wedge d \alpha$ be identically zero. We call $(M, \xi)$ a contact manifold if $\xi$ is completely nonintegrable in the sense that $\alpha \wedge d \alpha$ is nowhere zero. (This is clearly independent of the choice of $\alpha$.) Then $\alpha \wedge d \alpha$ determines an orientation on $M$ (independent of $\alpha$ and the orientation of $\xi$ ). The contact structure $\xi$ is called positive if this orientation agrees with the given one on $M$ and negative otherwise. Except where otherwise indicated, we will (without loss of generality) only deal with positive contact structures. A contactomorphism is a diffeomorphism preserving contact structures. According to Gray's Theorem [Gr], contact structures are all locally contactomorphic, and on closed manifolds they are deformation invariant in the sense that if a homotopy $\xi_{t}, 0 \leq t \leq 1$, of 2-plane fields on $M$ consists entirely of contact structures, then there is an isotopy $\varphi_{t}$ of $M$ with $\varphi_{0}=\operatorname{id}_{M}$, $\left(\varphi_{t}\right)_{*} \xi_{0}=\xi_{t}$ for each $t$, and $\varphi_{t}=\varphi_{0}$ wherever $\xi_{t}$ is independent of $t$. When this occurs, we say that $\xi_{0}$ and $\xi_{1}$ are isotopic. Note that contact structures that are homotopic as plane fields need not be homotopic through contact structures. It follows easily that an isotopy $\psi_{t}$ of $M$ that preserves $\xi$ for all $t$ on a subset $N$ can be changed rel $N$ to a contact isotopy, or isotopy of $M$ through contactomorphisms. (Apply Gray's Theorem to the family $\psi_{t}^{*} \xi$.)

A link $L: \coprod_{i=1}^{n} S^{1} \hookrightarrow M$ in a contact 3-manifold $(M, \xi)$ is called Legendrian [Ar] if its tangent vectors all lie in $\xi$. Two such links are considered equivalent if they are isotopic through a family of Legendrian links, or equivalently, if they are contact isotopic. Any link in a contact 3-manifold is $C^{0}$-small isotopic to a (nonunique) Legendrian link. (Simply replace each arc transverse to $\xi$ by a (left-handed) Legendrian spiral.) Any diffeomorphism $\varphi: K \rightarrow K^{\prime}$ between Legendrian knots extends to a contactomorphism on some neighborhoods of the knots. A Legendrian link comes equipped with a canonical framing of its normal bundle (up to fiber homotopy and orientation reversal), which is induced by any vector field transverse to $\xi$, or equivalently, by a vector field in $\xi \mid L$ transverse to $L$. This framing is preserved by contactomorphisms. For any nullhomologous knot $K$, there is a canonical bijection from (normal) framings of $K$ to the integers, sending each framing $f$ to the linking number of $K$ with its push-off determined by $f$, and in the Legendrian case the integer corresponding to the canonical framing of $K$ is called the Thurston-Bennequin invariant $t b(K)$. For any Legendrian knot $K$, we can find a $C^{0}$-small isotopy (necessarily changing its Legendrian knot type) that adds any number of left (negative) twists to the canonical framing - or in the nullhomologous case, decreases $t b(K)$ by any integer. (Simply add a spiral to $K$.) It is not always possible to add right twists (increase $t b(K)$ ), however. If $(M, \xi)$ admits a topologically unknotted Legendrian knot $K$ with $t b(K)=0$, then $\xi$ is overtwisted. (The reader can take this as a definition, or note that any disk bounded by $K$ is isotopic to an overtwisted disk.) In this case, we can add right twists to any canonical framing. Otherwise, $\xi$ is called tight, and any nullhomologous knot will have a Legendrian representative with maximal $t b$. For the unknot, the maximum will be -1 . There are knots in $S^{3}$, with its standard tight structure, for which the maximal $t b$ is arbitrarily large or small. (See [R1]; for $q=T B$, see [R2]). For any nullhomologous knot in a tight contact manifold there is a bound of $t b(K) \leq-\chi(F)$ for any embedded, orientable, connected surface $F$ bounded by $K$ [E5]. (See below for a sharper statement.)

The most interesting contact structures on 3-manifolds are the tight ones - they are somewhat analogous to taut foliations. While the classification of overtwisted contact structures on a closed 3manifold is simple (there is a unique such structure in each homotopy class of 2-plane fields [E1]), the occurrence of tight structures is poorly understood. Almost the only known obstruction to the existence of such structures $\xi$ is a bound on $c_{1}(\xi) \in H^{2}(M ; \mathbb{Z})$, the Chern class (or equivalently, Euler class) of $\xi$ as a complex line (real oriented 2-plane) bundle. Namely, if $F$ is a closed, connected, oriented surface in $M$, then $\left|\left\langle c_{1}(\xi), F\right\rangle\right| \leq-\chi(F)$ for $F \neq S^{2}$, and the left-hand side vanishes for $F=S^{2}$ [E4]. A primary source of tight contact structures is as follows: Consider a compact, complex surface $X$ with boundary $M$. Each tangent space $T_{x} M$ to $M$ will contain a unique 1-dimensional complex subspace of $T_{x} X$ (namely $\left.T_{x} M \cap i T_{x} M\right)$. If these complex lines comprise a contact structure $\xi$ on $M$ determining the boundary orientation, then $X$ has a strictly pseudoconvex boundary, and $(M, \xi)$ is called holomorphically fillable. 
Eliashberg [E3] proved that any holomorphically fillable contact 3-manifold is tight. This theorem and its symplectic generalization [E3] are the main tools available for proving tightness of contact structures. Note that if $M=\partial X$ and $\xi$ is the complex line field on $M$ induced by a complex (or almost-complex) structure $J$ on $X$, then the Chern class $c_{1}(\xi) \in H^{2}(M ; \mathbb{Z})$ is the restriction of that of $J$ on $X, c_{1}(J) \in H^{2}(X ; \mathbb{Z})$. This is because the complex bundle $T X \mid M$ splits as the sum of $\xi$ and a trivial complex line bundle.

There is one additional invariant known for Legendrian links. Let $L$ be a nullhomologous, oriented Legendrian link in $(M, \xi)$. (In practice, $L$ will be a knot.) Let $F$ be a Seifert surface for $L$, i.e., a compact, oriented surface embedded in $M$ (without closed components) whose oriented boundary is $L$. We define the rotation number $r(L, F)$ of $L$ with respect to $F$ to be the relative Chern number $\left\langle c_{1}(\xi, \tau), F\right\rangle$ of $\xi$ relative to a tangent vector field $\tau$ along $L$, evaluated on $F$. (Note that $c_{1}(\xi, \tau) \in H^{2}(M, L ; \mathbb{Z})$.) That is, we compute $r(L, F)$ by trivializing the 2-plane bundle $\xi \mid F$ and counting (with sign) how many times $\tau$ rotates in $\xi$ with respect to the trivialization as we travel around $L$. Clearly, $r(L, F)$ only depends on $F$ through its homology class in $H_{2}(M, L ; \mathbb{Z})$, and if $c_{1}(\xi)=0 \in H^{2}(M ; \mathbb{Z})$ then $r(L)=r(L, F)$ is independent of $F$. Note that $r(L, F)$ reverses sign if we reverse the orientation of either $L$ (hence, $F)$ or $\xi$. By adding left twists to $L$, one can realize any preassigned value of $r(L, F)$ at the expense of decreasing $t b$, as will be clear from our pictures below. In a tight contact manifold, the invariants $r$ and $t b$ classify those Legendrian knots that are topologically unknotted [EF], and for arbitrary nullhomologous Legendrian knots $K$ our previous bound on $t b(K)$ is sharpened by the inequality $t b(K)+|r(K)| \leq-\chi(F)$ [E5].

Our basic examples of contact 3-manifolds will be $\mathbb{R}^{3}, S^{3}$ and the connected sum $\# n S^{1} \times S^{2}$, each of which admits a unique (up to isotopy) tight contact structure compatible with its standard orientation [Be], [E4]. The structures on $S^{3}$ and $\# n S^{1} \times S^{2}$ are uniquely (up to blowups) holomorphically fillable - $S^{3}$ as the boundary of a round ball $B^{4}$ in $\mathbb{C}^{2}$, and $\# n S^{1} \times S^{2}$ as the boundary of $B^{4}$ union $n$ 1handles [E3]. To see the tight contact structure $\xi$ on $S^{3}$, we delete a point to obtain the tight structure on $\mathbb{R}^{3}$. We will always represent this as the kernel of the 1 -form $\alpha=d z+x d y$ on $\mathbb{R}^{3}$. (Note that $\alpha \wedge d \alpha=d x \wedge d y \wedge d z$.) We orient $\xi$ via the nowhere zero form $d \alpha|\xi=d x \wedge d y| \xi$. (Note that the contactomorphism $(x, y, z) \mapsto(x,-y,-z)$ reverses orientation on $\xi$.) We visualize $\left(\mathbb{R}^{3}, \xi\right)$ by projecting into the $y$ - $z$ plane. Then the plane $\xi_{(x, y, z)}$ at a point $(x, y, z)$ projects to a line at $(y, z)$ whose slope is $-x$.

Legendrian link theory in $S^{3}$ or $\mathbb{R}^{3}$ now reduces without loss of information to the theory of the corresponding front projections in $\mathbb{R}^{2}$, as developed by Arnol'd [Ar]. A Legendrian knot in $\mathbb{R}^{3}$ projects to a closed curve $\gamma$ in $\mathbb{R}^{2}$ that may have cusps and transverse self-crossings but has no vertical tangencies. (See Figure 1.) Any such curve comes from a unique Legendrian knot in $\mathbb{R}^{3}$, which may be reconstructed by setting $-x(t)$ equal to the slope of $\gamma$ at $t$, with cusps corresponding to points where the knot is parallel to the $x$-axis. Thus, at self-crossings, the curve of most negative slope always crosses in front. For example, Figure 1 represents the right-handed trefoil knot as in Figure 2. We will continue to draw overcrossings to avoid confusion, even though it is not actually necessary. Beware that the literature contains diagrams using the opposite convention, i.e., a left-handed coordinate system. In a front projection of a generic Legendrian link, the only singularities are transverse double points and cusps isotopic to the curves $z^{2}=y^{3}$ or $-y^{3}$. In analogy with the Reidemeister moves of ordinary link theory, the moves shown in Figure 3 (together with their images under $180^{\circ}$ rotation about any coordinate axis in $\mathbb{R}^{3}$ and isotopies 
of $\mathbb{R}^{2}$ introducing no vertical tangencies) suffice for realizing any equivalence of Legendrian links [S].

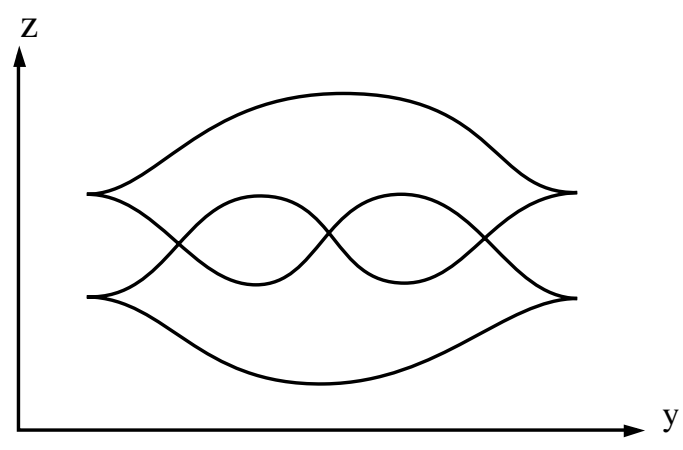

FigURE 1

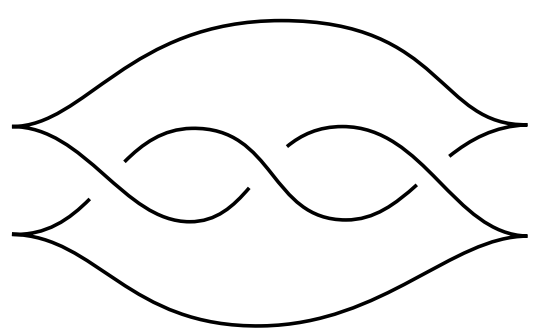

Figure 2

1)
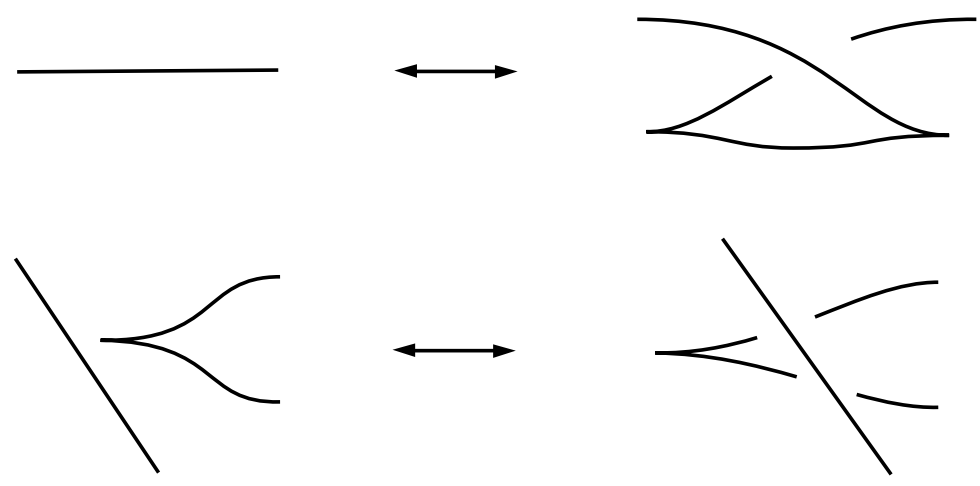

2)

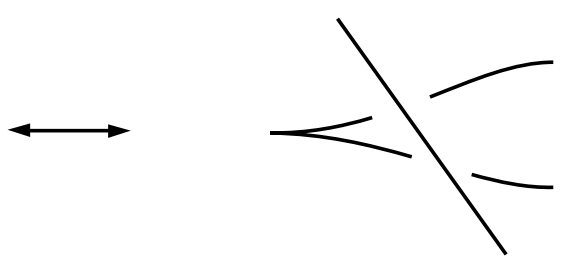

3)
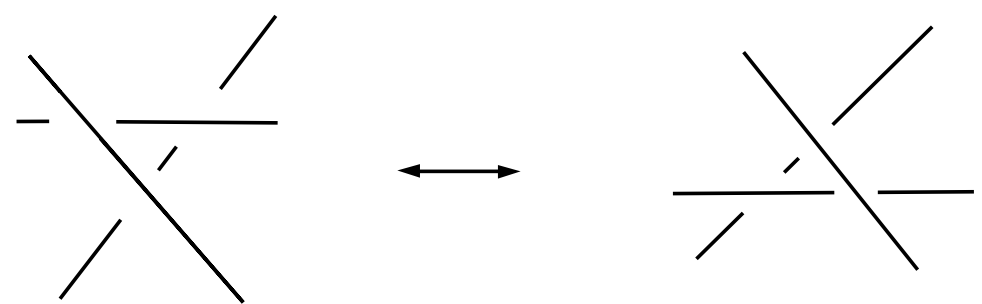

FIGURE 3

The invariants $t b(K)$ and $r(K)$ for an oriented Legendrian knot $K$ in $S^{3}$ or $\mathbb{R}^{3}$ are easy to compute from a front projection. (Note that these are always well-defined, since any knot is nullhomologous in $S^{3}$ or $\mathbb{R}^{3}$, and $c_{1}(\xi)=0$.) We begin with $t b(K)$. Any smooth planar diagram of a knot $K$ in $S^{3}$ determines an obvious blackboard framing via a normal vector field to the immersed curve in $\mathbb{R}^{2}$. This framing is not isotopy invariant. In fact, the integer corresponding to the blackboard framing is equal to the writhe $w(K)$ of $K$, which is the number of self-crossings of $K$ counted with sign. (Signs are determined by orienting $K$ arbitrarily and comparing with Figure 4, up to rotation.) 
$(+)$

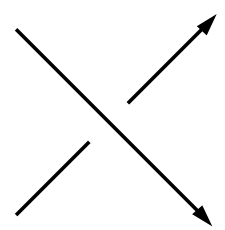

$(-)$

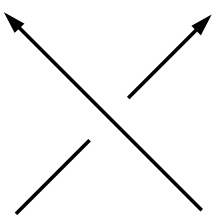

FiguRE 4

If $K$ is Legendrian, we can compute its canonical framing by observing that the vector field $\frac{\partial}{\partial z}$ on $\mathbb{R}^{3}$ is everywhere transverse to $\xi$, so it gives the canonical framing of $K$. This framing will agree with the blackboard framing (obtained by smoothing the projection of $K$ without adding crossings) except for a half left twist at each cusp (Figure 5).

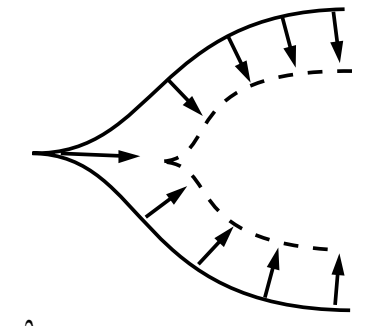

$\lambda$

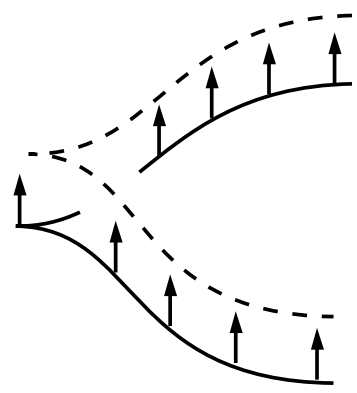

blackboard

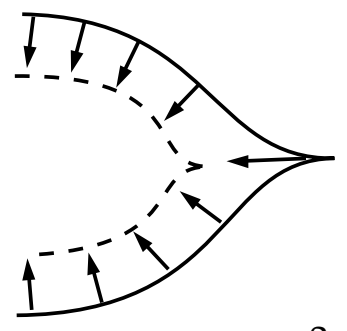

$\rho$

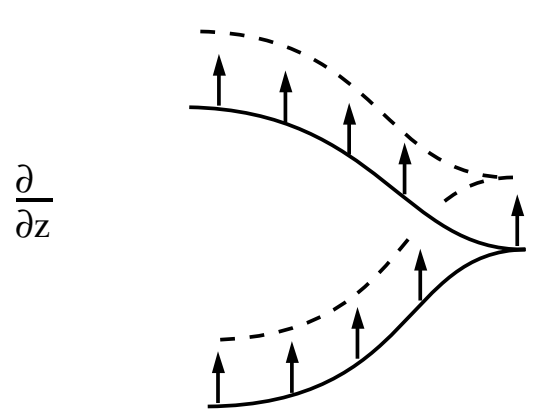

FIGURE 5

Thus, if $\lambda(K)$ (resp. $\rho(K))$ denotes the number of cusps with vertex on the left (resp. right) (Figure 5), we obtain that the canonical framing differs from the blackboard framing by $\frac{1}{2}(\lambda(K)+\rho(K))$ left twists. But clearly, $\lambda(K)=\rho(K)$, so we obtain

$$
t b(K)=w(K)-\frac{1}{2}(\lambda(K)+\rho(K))=w(K)-\lambda(K) .
$$

For example, the trefoil in Figure 2 has $t b=1$.

To compute the rotation number $r(K)$, we observe that the vector field $\frac{\partial}{\partial x}$ trivializes the 2-plane bundle $\xi$ on $\mathbb{R}^{3}$, so it restricts to a trivialization of $\xi \mid F$ for any Seifert surface $F$. Thus, it suffices to count (with sign) how many times the tangent vector field $\tau$ of $K$ crosses $\frac{\partial}{\partial x}$ as we travel around $K$. Let $\lambda_{+}(K)$ (resp. $\left.\lambda_{-}(K)\right)$ be the number of left cusps at which $K$ is oriented upward (resp. downward), and define $\rho_{ \pm}(K)$ similarly (Figure 6$)$. Let $t_{ \pm}=\lambda_{ \pm}+\rho_{ \pm}$be the total number of upward $\left(t_{+}\right)$and downward $\left(t_{-}\right)$cusps. Since each downward left cusp represents a positive crossing of $\tau$ past $\frac{\partial}{\partial x}$ (counterclockwise with respect to $d x \wedge d y$ ) and each upward right cusp represents a negative crossing, we obtain

$$
r(K)=\lambda_{-}-\rho_{+}=\rho_{-}-\lambda_{+}=\frac{1}{2}\left(t_{-}-t_{+}\right),
$$


where the second equality is obtained by using $-\frac{\partial}{\partial x}$ instead of $\frac{\partial}{\partial x}$ and the third is obtained by averaging the first two. Note that reversing the orientation of $K$ reverses the sign of $r(K)$, as required. If we add $k>0$ upward (downward) zig-zags to $K$ as in Figure 7 (with $k=3$ ), the effect will be to decrease $t b(K)$ by $k$ and decrease (increase) $r(K)$ by $k$.
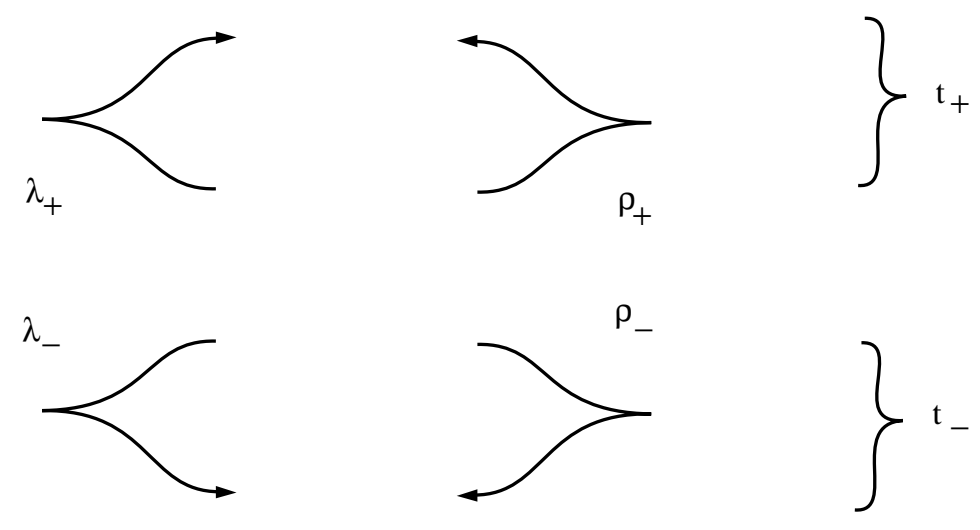

FIGURE 6

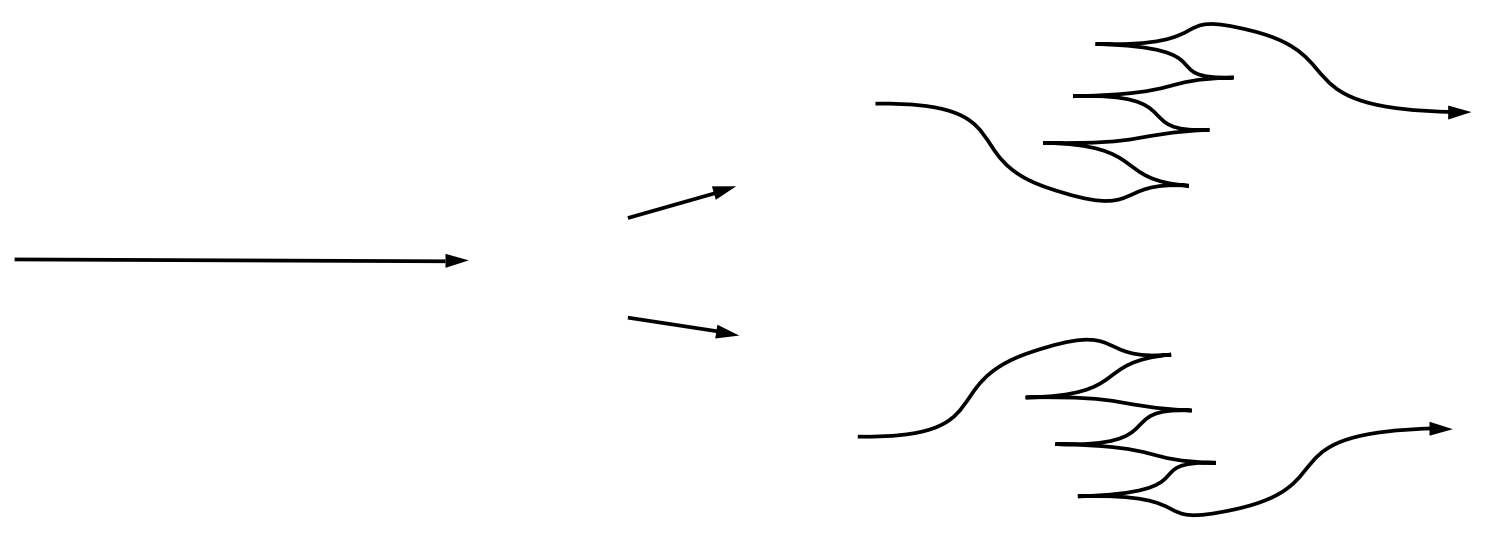

FIGURE 7

The main purpose of this discussion was to prepare for the statement and applications of Eliashberg's Theorem in dimension 4. Recall [K], [GS] that a handle decomposition of a compact, oriented 4-manifold $X$ with all handles of index $\leq 2$ is equivalent to a framed link in $\# n S^{1} \times S^{2}=\partial$ (0-handle $\cup$ 1-handles $)$. For each link component, we attach a 2-handle $D^{2} \times D^{2}$ along $S^{1} \times D^{2}$, by identifying the link component with $S^{1} \times 0$ and its framing with the product framing on $S^{1} \times 0 \subset S^{1} \times D^{2}$. If the attaching circle is nullhomologous in $\# n S^{1} \times S^{2}$, then its framing is specified by an integer, which is also the self-intersection number $\alpha^{2}(=\alpha \cdot \alpha)$ of the homology class $\alpha \in H_{2}(X ; \mathbb{Z})$ determined (up to sign) by the 2-handle. For noncompact 4-manifolds we obtain a similar description, although it can be more complicated due to the end structure of the union of 0- and 1-handles. For Stein surfaces, we have the following theorem, which is implicit in [E2]. (See also [E5].)

Theorem 1.3 (Eliashberg). A smooth, oriented, open 4-manifold $X$ admits a Stein structure if and only if it is the interior of a (possibly infinite) handlebody such that the following hold:

(a) Each handle has index $\leq 2$,

(b) Each 2-handle $h_{i}$ is attached along a Legendrian curve $K_{i}$ in the contact structure induced on the boundary of the underlying 0 - and 1-handles, and 
(c) The framing for attaching each $h_{i}$ is obtained from the canonical framing on $K_{i}$ by adding a single left (negative) twist.

A smooth, oriented, compact 4-manifold $X$ admits a Stein structure if and only if it has a handle decomposition satisfying $\mathrm{a}, \mathrm{b}$ and $\mathrm{c}$. In either case, any such handle decomposition comes from a strictly plurisubharmonic function (with $\partial X$ a level set).

Thus, the previous discussion gives an explicit procedure for constructing Stein surfaces with strictly plurisubharmonic Morse functions lacking index 1 critical points - Simply add 2-handles to a Legendrian link in $S^{3}=\partial B^{4}$ such that the framing on each $h_{i}$ is given by $t b\left(K_{i}\right)-1$. We deal with general Stein surfaces in the next section.

Eliashberg proves this theorem by explicit holomorphic gluing. Each 2-handle is given as a neighborhood of $D^{2} \times 0 \subset i \mathbb{R}^{2} \times \mathbb{R}^{2}=\mathbb{C}^{2}$. (Note that the equality reverses the natural orientations.) The attaching circle $S^{1} \times 0$ is glued to the given Legendrian curve. In particular, the unit tangent vector field $\tau$ to $S^{1} \times 0$ is mapped into $\xi$. Since $\xi$ now represents a complex line field, $i \tau$ must also map into $\xi$, so $i \tau$ goes to the canonical framing. However, $i \tau$ differs from the product framing on $S^{1} \times \mathbb{R}^{2}$ by one twist, since $\tau: S^{1} \rightarrow S^{1}$ has degree 1. This accounts for the difference of one twist between the canonical framing and the attaching framing of the 2-handle. (The twist is left-handed because of the above-mentioned orientation reversal.)

The Chern class of a Stein surface $X$ without 1-handles is easy to compute from a Legendrian link diagram (cf. [E5]). The homology $H_{2}(X ; \mathbb{Z})$ will be free abelian. To choose a basis, one orients the framed link. Then each link component is the oriented boundary of a Seifert surface whose union with the (suitably oriented) core of the 2-handle represents a basis element. The Chern class $c_{1}(X)$ can now be characterized as the unique element of $H^{2}(X ; \mathbb{Z})$ whose value on each such basis element is the rotation number of the corresponding oriented Legendrian link component. We will prove a more general statement in the next section (Proposition 2.3).

\section{1-handles.}

In order to represent arbitrary Stein surfaces by means of Legendrian link diagrams, we must find a way to represent 1-handles. In the usual Kirby calculus for representing handlebodies via framed links $[\mathrm{K}]$, [GS], one can represent a 1-handle by drawing its attaching region. This will be a pair of 3-balls $B_{1}$ and $B_{2}$ in $S^{3}=\partial B^{4}$ (represented by $\mathbb{R}^{3}$ ), which are taken to be identified by a diffeomorphism $\varphi: B_{1} \rightarrow B_{2}$ that reverses orientation if the 4-manifold is orientable. Without loss of generality, we can take $B_{1}$ and $B_{2}$ to be round, and choose $\varphi$ conveniently. The standard convention is to take $\varphi$ to be reflection through the plane perpendicularly bisecting the line segment connecting the centers of $B_{1}$ and $B_{2}$. It is sometimes convenient to put the balls in a standard position and assume (without loss of generality) that the attaching curves of the 2-handles lie in the region between the balls. For example, this is convenient if we wish to specify framings by integers, as will be discussed below.

We will find a similar description of 1-handles in the setting of Stein surfaces, and establish a standard form for Legendrian links in $\# n S^{1} \times S^{2}$ that allows us to conveniently define and compute ThurstonBennequin invariants. We must observe, however, that the diffeomorphism $\varphi$ is necessarily more complicated than in the smooth case. To understand this, we must consider the characteristic foliation of a surface $F$ in a contact 3-manifold $(M, \xi)$, which is the singular foliation on $F$ induced by the singular field of lines $\xi_{x} \cap T F_{x}$ on $F$. The characteristic foliation of a small round sphere in $\mathbb{R}^{3}$, centered in the $y-z$ plane, has two singular points, one at each pole. In between, the foliation is roughly a left-handed spiral with infinitely many turns near each pole. The spirals are somewhat twisted, however, so that they always cross the $y-z$ plane orthogonally to it. If gluing $B_{1}$ and $B_{2}$ by $\varphi$ is to produce a manifold with contact boundary, then $\varphi$ must preserve the characteristic foliations on $\partial B_{j}$. If the contact plane field is to be orientable, then $\varphi$ must interchange the poles of the spheres $\partial B_{j}$ (so that an inward $\frac{\partial}{\partial z}$ maps to an outward one). Since $\varphi$ reverses orientation, the spheres $\partial B_{j}$ cannot both be round, for the left-handed spiral of $\partial B_{j}$ at each pole would necessarily map to a right-handed spiral. This is easily remedied by a

$C^{1}$-small perturbation near each pole to make $\partial B_{j}$ look locally like the saddle $z=-\frac{1}{2} x y$ at the origin, up to translation (cf. [E4]). The characteristic foliations will then be radial near the poles, so it will be possible to define a map $\varphi$ preserving the characteristic foliations. Note that an additional twist will be 
required to match up the spirals away from the poles.

Fortunately, we will see that in the end it is not necessary to understand $\varphi$ in detail. We will align $B_{1}$ and $B_{2}$ along the same horizontal line, show that we can assume $\varphi$ identifies the two points $p_{j} \in B_{j}$ that are closest to each other, and see that it suffices to keep track of $\varphi$ in a neighborhood of each $p_{j}$. Note that at each $p_{j}$, the characteristic foliation will have tangent $\pm \frac{\partial}{\partial x}$. If we orient these flow lines to point toward us $\left(+\frac{\partial}{\partial x}\right)$, they will continue toward opposite poles of $B_{1}$ and $B_{2}$. Thus, $\varphi$ will preserve the direction $\frac{\partial}{\partial x}$ at $p_{j}$, which will allow us to approximate $\varphi$ by the usual reflection in a neighborhood of $p_{1}$. (Other conventions are possible - for example, we could align $B_{1}$ and $B_{2}$ vertically and identify their nearest poles by translating the saddle-shaped regions. This seems to complicate the resulting Legendrian link diagrams, however, since one must deal carefully with Legendrian curves passing through the saddles.)

Definition 2.1. A Legendrian link diagram in standard form, with $n \geq 0$ 1-handles, is given by the following data (see Figure 8):

1) A rectangular box parallel to the axes in $\mathbb{R}^{2}$,

2) A collection of $n$ distinguished segments of each vertical side of the box, aligned horizontally in pairs and denoted by balls, and

3) A front projection of a generic Legendrian tangle (i.e., disjoint union of Legendrian knots and arcs) contained in the box, with endpoints lying in the distinguished segments and aligned horizontally in pairs.

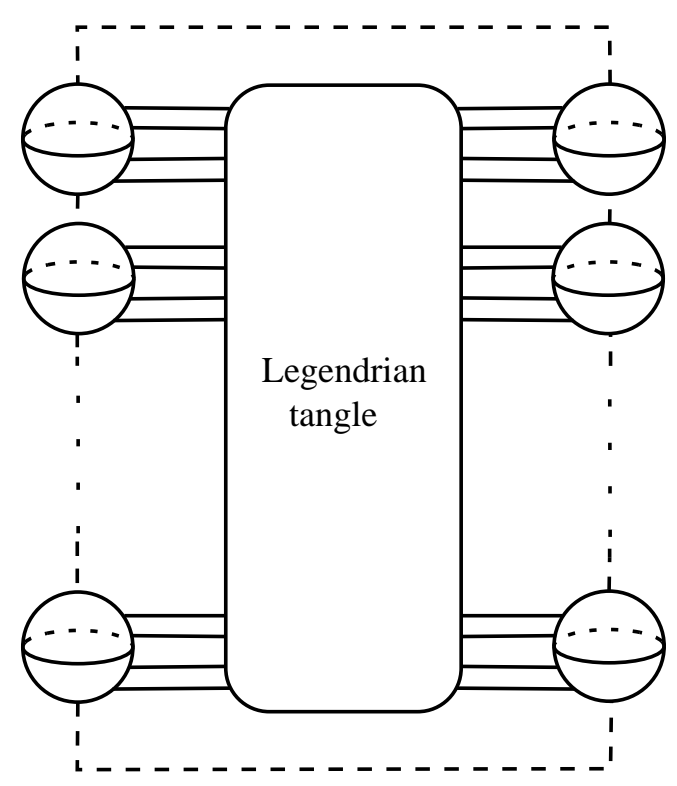

FiguRe 8

Thus, if we attach 1-handles to the pairs of balls, we will obtain a link in $\# n S^{1} \times S^{2}$. Now let $H$ denote a handlebody consisting of a 0 -handle and $n$ 1-handles, with the canonical Stein structure determined by Theorem 1.3 and contact structure $\xi$ on $\partial H$. Fix an ordering of the 1-handles, a direction for each 1 -handle and a homotopy class of nowhere zero vector fields in $\xi$. Such vector fields exist because $c_{1}(\xi)$ is the restriction of $c_{1}(H) \in H^{2}(H ; \mathbb{Z})=0$.

Theorem 2.2. The boundary of $H$ can be identified with a contact manifold obtained from the standard contact structure on $S^{3}$ by removing smooth balls and gluing the resulting boundaries as in Figure 8. The identification exhibits $H$ in the usual way as a smooth handlebody, and it can be assumed to match the above data for $H$ with corresponding preassigned data for the diagram. Any Legendrian link in $\partial H=\# n S^{1} \times S^{2}$ is contact isotopic to one in standard form. Two Legendrian links in standard form are 
contact isotopic in $\partial H$ if and only if they are related by a sequence of the six moves shown in Figures 3 and 9 (and their images under $180^{\circ}$ rotation about each axis), together with isotopies of the box that fix the boundary outside of the balls and introduce no vertical tangencies.

4)

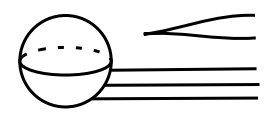

5)
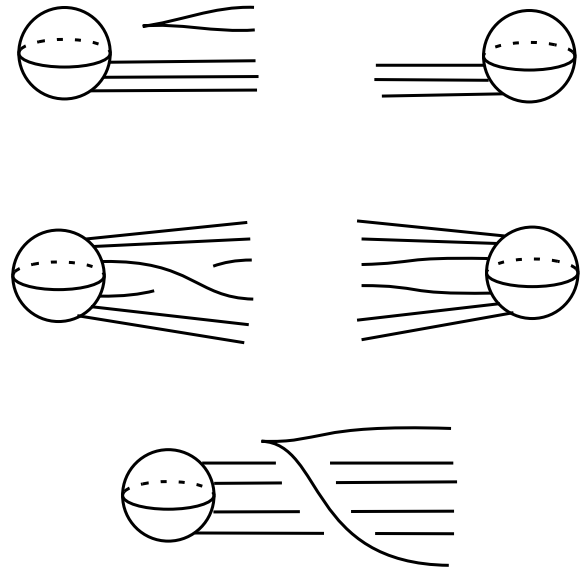
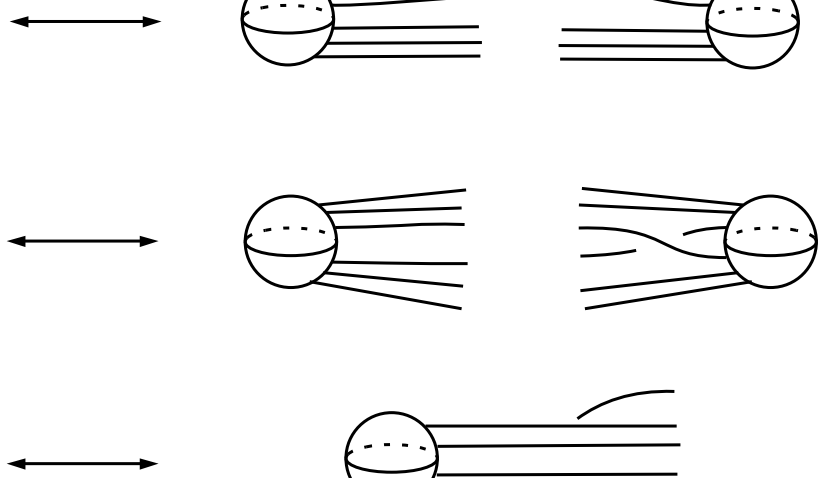

FIGURE 9
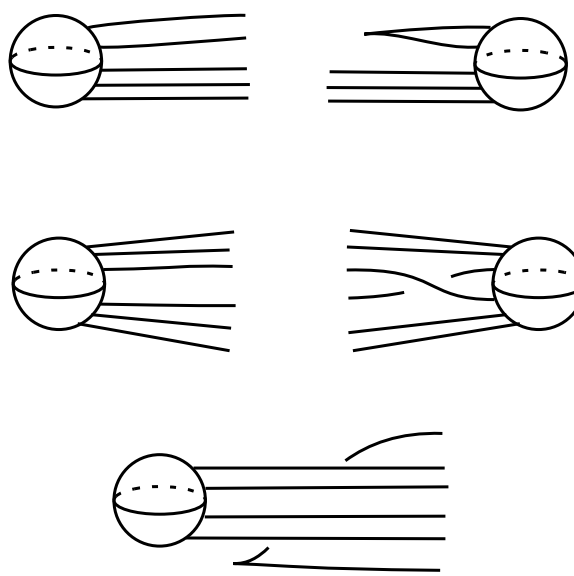

The new moves correspond to sliding cusps and crossings over 1-handles, and to swinging a strand around an attaching ball of a 1-handle. We prove the theorem at the end of this section. Following the proof, we show that different homotopy classes of vector fields result in different descriptions of $H$. These will be contactomorphic, and we describe the contactomorphisms explicitly, but no such contactomorphism will be contact isotopic to the identity. For a sample of the difficulties encountered in proving the theorem, consider how to put a link in standard form if it contains Figure 10 - Beware of disallowed crossings.

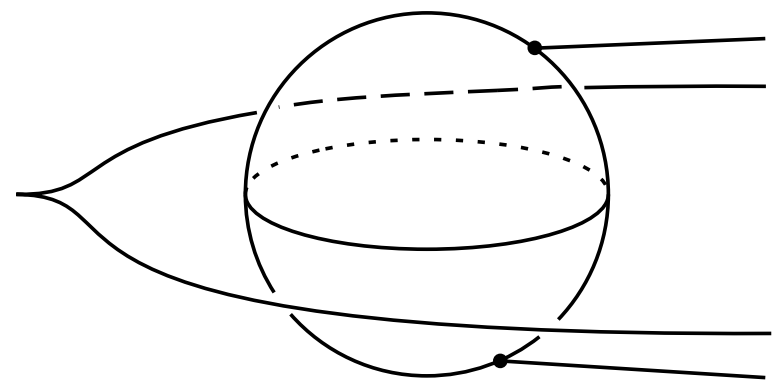

FigURE 10

For arbitrary knots in $\# n S^{1} \times S^{2}$, we would like to establish a convention for identifying framings with integers, just as we did using linking numbers in the nullhomologous case. There is no isotopy-invariant way to do this. However, we can adopt a convention from Kirby calculus that assigns integers to framed knots that are in standard form. These integers will be invariant under isotopies within the box of Definition 2.1, i.e., Moves 1-5 (Figures 3, 9), but will change under Move 6. To establish the convention, simply stretch the box in the plane and glue its lateral edges together so that it becomes an annulus, identifying endpoints of the tangle in the natural way. The tangle becomes an actual knot projected into the plane. Now we can identify framings with integers using our previous convention. As before, the blackboard framing becomes $w(K)$, the signed number of self-crossings of $K$. Clearly, this definition has the required isotopy invariance. It also agrees with the previous one in the nullhomologous case. Move 6 changes the integer corresponding to any framing on $K$ by twice the number of times (counted with sign) 
that $K$ runs over the 1-handle. To check this and fix the sign, simply consider the blackboard framing, which is invariant under the move. Now for $K$ Legendrian, we define $t b(K)$ as before, as the integer corresponding to the canonical framing. It follows immediately that $t b(K)$ is still given by Formula 1.1. Note that $t b(K)$ can change under Move 6, even though the canonical framing itself is invariant under contactomorphisms.

We must also define the rotation number $r(K)$ in more generality. Suppose that $K$ is an oriented Legendrian knot in a contact 3-manifold $(M, \xi)$ with $c_{1}(\xi)=0$. Thus, as an abstract real 2-plane bundle, $\xi$ is trivial. For any preassigned choice of nowhere zero vector field $v$ in $\xi$ (up to homotopies through such vector fields) we can define the rotation number $r_{v}(K)$ to be the signed number of times that the tangent vector field $\tau$ to $K$ rotates in $\xi$ relative to $v$ as we traverse $K$. In the nullhomologous case, $v$ determines a trivialization of $\xi \mid F$ on any Seifert surface $F$ for $K$, so our new definition agrees with the old one (and hence is independent of $v$ ) in this case. Now for the tight contact structure $\xi$ on $\# n S^{1} \times S^{2}$, we choose $v$ to be $\frac{\partial}{\partial x}$ everywhere in the box in Definition 2.1. This fits together in the obvious way on the 1-handles, and then extends uniquely over $\# n S^{1} \times S^{2}$. (Such vector fields are classified by $H^{1}(\cdot ; \mathbb{Z})$.) Now $r(K)=r_{v}(K)$ is well-defined for any oriented Legendrian knot $K$ in our diagram of $\# n S^{1} \times S^{2}$, and it is invariant under contact isotopy. Note, however, that Theorem 2.2 allows us to identify the diagram with $H$ so that $v$ corresponds to any preassigned nowhere zero vector field in $\xi$ in $\partial H$. Changing the latter vector field will change rotation numbers of Legendrian knots in $\partial H$. The resulting nontrivial contactomorphisms of $\partial H$ will be described explicitly at the end of this section. Our previous argument shows that $r(K)$ can still be computed via Formula 1.2 when $K$ is in standard form. In Section 4 we will show that $t b(K)+r(K)+1$ is always congruent mod 2 to the number of times $K$ crosses 1-handles (Corollary 4.13).

We are led to the following characterization of compact Stein surfaces with boundary.

Proposition 2.3. A smooth, oriented, compact, connected 4-manifold $X$ admits the structure of a Stein surface (with boundary) if and only if it is given by a handlebody on a Legendrian link in standard form (Definition 2.1) with the $i^{\text {th }}$ 2-handle $h_{i}$ attached to the $i^{\text {th }}$ link component $K_{i}$ with framing $t b\left(K_{i}\right)-1$ (as given by Formula 1.1). Any such handle decomposition is induced by a strictly plurisubharmonic function. The Chern class $c_{1}(J) \in H^{2}(X ; \mathbb{Z})$ of such a Stein structure $J$ is represented by a cocycle whose value on each $h_{i}$, oriented as at the end of Section 1, is $r\left(K_{i}\right)$ (as given by Formula 1.2).

Proof. The first part of this proposition is just Eliashberg's theorem (1.3) augmented by Theorem 2.2 and the subsequent discussion. (To obtain a unique 0-handle, apply the connectedness of $X$ and uniqueness of fillings of $S^{3}$.) To compute $c_{1}(J)$, we trivialize $T X$ as a complex bundle over the union $X_{1}$ of the 0 and 1-handles, and measure the failure of the trivialization to extend over the 2-handles. We trivialize $T X$ over the box given in Definition 2.1 (as a subset of $X_{1}$ ), by using the vector field $\frac{\partial}{\partial x}$ (which spans $\xi$ ) and an inward normal to $\partial X_{1}$. These extend uniquely to a frame field $(u, v)$ that trivializes $T X_{1}$ (since $\left.X_{1} \simeq \vee S^{1}\right)$. Now recall that each 2-handle $h_{i}$ is a neighborhood of $D^{2} \times 0 \subset i \mathbb{R}^{2} \times \mathbb{R}^{2}$. We choose a convenient trivialization of its tangent bundle. The tangent and outward normal vector fields $\tau$ and $\nu$ to $S^{1} \subset D^{2}$ together trivialize $T D^{2} \mid S^{1}$ over $\mathbb{R}$. The frame field $(\tau, \nu)$ differs from the product frame field on $D^{2} \subset i \mathbb{R}^{2}$ by an element of $\pi_{1}(S O(2))$. Since $D^{2}$ lies in $i \mathbb{R}^{2} \subset \mathbb{C}^{2}$, the field $(\tau, \nu)$ also forms a complex trivialization of $T h_{i} \mid S^{1}$, which differs from a product trivialization by the same element of $\pi_{1}(S O(2))$. Since $S O(2) \subset S U(2) \subset U(2)$ and $S U(2)$ is simply connected, $(\tau, \nu)$ extends to a complex trivialization $\left(\tau^{*}, \nu^{*}\right)$ over all of $h_{i}$. When we attach $h_{i}$ to $X_{1}, \tau$ is identified with a tangent vector field to $K_{i}$ and $\nu$ becomes inward normal to $\partial X_{1}$ in $T X_{1}$. Clearly, $\tau^{*}$ and $u$ span a complex line bundle $L$ (which agrees with $\xi$ in the box), while $\nu^{*}$ and $v$ can be fit together to span a complementary trivial line bundle. Thus, the desired cochain evaluated on $h_{i}$ is just the relative Chern number of $L$, which is given by the rotation number of $\tau$ in $\xi$ relative to $\frac{\partial}{\partial x}$, or $r\left(K_{i}\right)$. (To check the sign, recall that in the nullhomologous case, $r\left(K_{i}\right)$ was defined as a relative Chern number on a Seifert surface for $K_{i}$, so it is $c_{1}(L)$ evaluated on the corresponding closed surface in $X$.)

Note that the cochain specified above depends on our choice of the vector field used to define $r\left(K_{i}\right)$, and hence, on how we chose to represent $X_{1}$ as a picture. However, the cohomology class is well-defined, since changing the vector field modifies the cocycle by a coboundary. Clearly, the above procedure also 
yields $c_{1}(J)$ for Stein surfaces $(X, J)$ with infinite topology (where we define the rotation numbers $r\left(K_{i}\right)$ relative to any convenient vector field). Alternatively, note that while an infinite handle decomposition may have a more complicated link diagram than we have considered (due to the end-structure of the union of 0- and 1-handles), any finite subhandlebody can be put in standard form.

Before proving Theorem 2.2, it will be convenient to introduce another model for $S^{1} \times S^{2}$. We begin by identifying $S^{1} \times B^{3}$ with a closed $\varepsilon$-neighborhood, with $\varepsilon=1$, of $0 \times i \mathbb{R}$ in $\mathbb{C}^{2}$, modulo translations by $0 \times 2 \pi i \mathbb{Z}$. The tight contact structure of complex lines tangent to $S^{1} \times S^{2}$ will be given by the 1 -form $u_{1} d u_{2}-u_{2} d u_{1}+u_{3} d u_{4}$ on $S^{1} \times S^{2}$, in the coordinates $\left(u_{1}+i u_{2}, u_{3}+i u_{4}\right)$ on $\mathbb{C}^{2}$. We pull this form back to $\mathbb{R}^{3}$ with cylindrical coordinates $(r, \theta, t)$, via stereographic projection from a plane through the origin, $\mathbb{R}^{2} \times \mathbb{R} \rightarrow\left(S^{2}-(0,0,-1)\right) \times \mathbb{R} \subset \mathbb{C}^{2}$, obtaining the form $d \theta+\frac{1-r^{4}}{4 r^{2}} d t$ after rescaling the form by a positive function. This pulls back to the standard form $\alpha=d z+x d y$ on $\mathbb{R}^{3}$, via the covering map from $\mathbb{R}^{3}$ to $\mathbb{R}^{3}$ minus the $t$-axis given by setting $x=\frac{1-r^{4}}{4 r^{2}}, y=t, z=\theta$. Thus, we have exhibited the standard contact structure on $S^{1} \times\left(S^{2}-\right.$ ppoles $\left.\}\right)$ as being the standard structure $\left(\mathbb{R}^{3}, \xi\right)$ modulo translations by $2 \pi(\mathbb{Z} \times \mathbb{Z})$ in the $y$ - $z$ plane. The spheres $p \times S^{2}$ are given by the annuli $y=$ constant (modulo translations in the $z$-direction), each compactified by two points (at $x= \pm \infty$ ). Now we can represent Legendrian links in $S^{1} \times S^{2}$ in the usual way by their front projections into $\mathbb{R}^{2} / 2 \pi \mathbb{Z}^{2}$. The new move corresponding to Move 6 (Figure 9) is given by Figure 11 and its image under $180^{\circ}$ rotation about the $y$-axis (corresponding to pushing the link through the transverse circles at $x= \pm \infty$ ). Note that we can use these moves to make a link disjoint from the top and bottom edges of the square; the correspondence with Theorem $2.2(n=1)$ should now be clear.
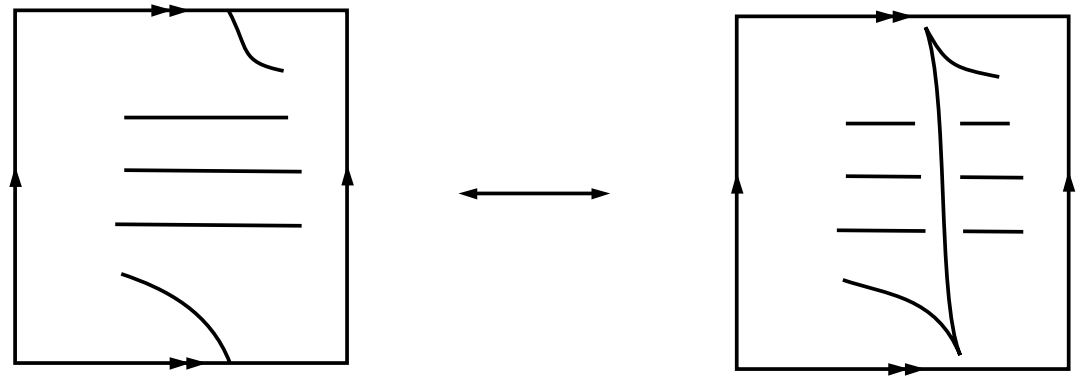

FIGURE 11

Proof of Theorem 2.2. First, we show that an arbitrary embedding $f: S^{2} \rightarrow M$ into a tight contact 3-manifold can be perturbed so that its image $f\left(S^{2}\right)$ has a neighborhood contactomorphic to that of $p \times S^{2} \subset S^{1} \times S^{2}$. By [Gi1], it suffices to perturb $f$ so that $f\left(S^{2}\right)$ has the same characteristic foliation as $p \times S^{2}$. Tightness guarantees that the foliation on $f\left(S^{2}\right)$ has no closed leaves (which would be Legendrian unknots with $t b=0$ ). By [E3] (see also [E5]), we can make a $C^{0}$-small perturbation of $f$ so that $f\left(S^{2}\right)$ has only two singular points in its characteristic foliation. By a $C^{1}$-small perturbation near the singular points, we can arrange the characteristic foliation to be radial there (cf. [E4]), so that it is globally determined (up to diffeomorphisms of $S^{2}$ ) by its monodromy map between the circles of unit tangent vectors at the singular points. By an additional $C^{1}$-small perturbation near one singular point, we can change this monodromy by a diffeomorphism of $S^{1}$, so that the characteristic foliation agrees with that of $p \times S^{2}$ in $S^{1} \times S^{2}$ as required. To see this, consider the local model of the singularity given by one pole of $p \times S^{2}$ in $S^{1} \times S^{2}$, or in our model of the latter, the $x-z$ plane in the region $x \geq N,|y|<\varepsilon$ in $\left(\mathbb{R}^{3}, \xi\right) / 2 \pi \mathbb{Z}^{2}$. In the annulus between the circles $y=0$ and $y=\varepsilon / 2$ in the $y$ - $z$ plane modulo $0 \times 2 \pi \mathbb{Z}$, draw a flow realizing the given diffeomorphism of $S^{1}$. By composing with additional $2 \pi$-rotations, we may assume the flow lines have arbitrarily negative slope everywhere, and that the slopes increase in absolute value with increasing $y$ for fixed $z$. Interpreting these flow lines as Legendrian curves in $\mathbb{R}^{3}$, we obtain a surface near the $x-z$ plane with the required characteristic foliation. (To make the perturbation $C^{1}$-small, identify $p \times S^{2}$ locally with the plane $z=0$ in $\left(\mathbb{R}^{3}, d z+r^{2} d \theta\right)$ and rescale by $(r, z) \mapsto\left(t r, t^{2} z\right)$.)

Now given $H$ with its ordered collection of $n$ 1-handles, let $S_{1}, \ldots, S_{n}$ be the corresponding belt spheres. As above, we can assume that a neighborhood of $p \times S^{2}$ maps contactomorphically into $\partial H$ with 
$p \times S^{2}$ mapping to $S_{i}$. Similarly, we can find such a map into $\mathbb{R}^{3}$, and the composite realizes $S_{i}$ (with both choices of orientation) as the boundary of a contact 3 -ball $B \subset \mathbb{R}^{3}$. We can choose $B$ to be nearly round, with $0 \in \mathbb{R}^{3}$ lying on the equator of $\partial B$, so that each contactomorphism $\psi_{t}(x, y, z)=\left(t x, t y, t^{2} z\right)$, $0<t \leq 1$, sends $B$ into itself fixing 0 . Now we can cut open $\partial H$ along each $S_{i}$ and glue in a pair of copies $B_{i 1}$ and $B_{i 2}$ of $B$, with the second index determined by the direction of the 1-handle. The result is $S^{3}$ with its unique tight contact structure. (An overtwisted disk could be isotoped off of the balls $B_{i j}$ using $\psi_{t}$, contradicting the tightness of $\partial H$.) Choose nonsingular points $p_{i j} \in \partial B_{i j}$ such that $p_{i 1}$ and $p_{i 2}$ correspond under the gluing map $\varphi_{i}: \partial B_{i 1} \rightarrow \partial B_{i 2}$ for $\partial H$. We can assume (after a contact isotopy of $\mathbb{R}^{3}$ fixing $\partial B$ setwise) that each identification $B_{i j} \approx B$ sends $p_{i j}$ to 0 . After a contact isotopy of $S^{3}$, we can assume that the points $p_{i j}$ are arranged along the edges of a box, say $p_{i j}=(0, j, i) \in \mathbb{R}^{3}$, with the outward normals to $B_{i j}$ at $p_{i j}$ directed into the box and $\left(d \varphi_{i}\right)_{p_{i 1}}=\mathrm{id}_{\mathbb{R}^{2}}$. By conjugating the contact isotopy $\psi_{t}, \varepsilon \leq t \leq 1$, with each identification $B_{i j} \approx B$, and extending to a contact isotopy of $S^{3}$ via Gray's Theorem, we can arrange for the balls $B_{i j}$ to be embedded in $\mathbb{R}^{3}$ by nearly linear maps. (We draw them as round balls although they will actually be ellipsoidal.) Since $\left(d \varphi_{i}\right)_{p_{i 1}}=\mathrm{id}_{\mathbb{R}^{2}}$, we can find connected neighborhoods $U_{i j}$ of $p_{i j}$ in $\partial B_{i j}$ on which the gluing map $\varphi_{i}: U_{i 1} \rightarrow U_{i 2}$ is arbitrarily closely approximated by a translation in $\mathbb{R}^{3}$. If we apply a contactomorphism of $S^{3}$ supported near $p_{i 1}$, rotating the tangent space $360^{\circ}$ about a vertical axis, before shrinking $B_{i 1}$ with $\psi_{t}$, the vector field in $\partial H$ corresponding to $\frac{\partial}{\partial x}$ in the box will be changed by a full twist. Since nowhere zero vector fields in $\xi$ are classified by $H^{1}(\partial H ; \mathbb{Z})$, the vector field corresponding to $\frac{\partial}{\partial x}$ can be chosen arbitrarily. We have now represented $H$ as in Definition 2.1, with the required control of the auxiliary data.

Now let $L$ be a Legendrian link in $\partial H$. The image of $L$ in $S^{3}$ will be a tangle that can run into, around, behind or in front of the sphere $S_{i j}=\partial B_{i j}$ as in Figure 10, although we can assume that the front projection of $L$ is disjoint from each $p_{i j}$, and hence (after we shrink the neighborhoods $U_{i j}$ ) the projections of $L$ and $U_{i j}$ are disjoint. For each $i$, let $A_{i} \subset S_{i}$ be the arc whose interior is a leaf of the characteristic foliation, and whose image in $S_{i 1}$ contains $p_{i 1}$. By general position, we can assume that $L$ is disjoint from each $A_{i}$. Using our contactomorphism on a neighborhood of $S_{i}$ sending $S_{i}$ to $p \times S^{2} \subset S^{1} \times S^{2}$, we can visualize $S_{i}-A_{i}$ as $\mathbb{R} \times 0 \times(0,2 \pi) \subset \mathbb{R} \times(-\varepsilon, \varepsilon) \times \mathbb{R} / 2 \pi \mathbb{Z}$ in our picture of $S^{1} \times S^{2}$. For any compact subset $C$ of $\partial H-A_{i}$, there is a contact isotopy $F_{s}$ compactly supported in $\mathbb{R} \times(-\varepsilon, \varepsilon) \times(0,2 \pi)$ that moves $C$ to a set whose intersection with $S_{i}$ maps into $U_{i 1}$ in $S_{i 1}$. To construct this, start with a suitable isotopy $f_{s}$ in $(-\varepsilon, \varepsilon) \times(0,2 \pi)$ preserving the direction of $\frac{\partial}{\partial z}$ (see Figure 12). There is a unique contact isotopy of $\mathbb{R} \times(-\varepsilon, \varepsilon) \times(0,2 \pi)$ projecting to $f_{s}$, since the $x$-coordinate of each point is determined by the slope of the corresponding contact plane, hence controlled by $d f_{s}$. To obtain compact support, truncate the isotopy for sufficiently large values of $|x|$ by applying Gray's Theorem. Taking $C=L$, we isotope $L$ so that it intersects the spheres $S_{i j}$ only in $U_{i j}$. By prechoosing $U_{i j}$ and $\varepsilon$ to be sufficiently small, we can assume that all points in our neighborhood of $S_{i}$ that project onto $U_{i j}$ in our original planar diagram of $\partial H$ (Definition 2.1) will lie in a preassigned neighborhood of the top and bottom edges of Figure 12, with a narrow range of $x$-coordinates. Thus, the only points of $L$ that may project onto $U_{i j}$ (after the above isotopy of $L$ ) will lie on small arcs intersecting $S_{i}$ in Figure 12. In the original diagram, these arcs will be parallel, emerging horizontally from $S_{i j}$ and then doubling back with nowhere zero slope to follow the characteristic folation of $S_{i j}$. By increasing the slope if necessary, we can arrange that $L$ project onto $U_{i j}$ only at the endpoints of the tangle. Let $\ell_{i}$ denote the horizontal line in $\mathbb{R}^{3}$ passing through the centers of the balls $B_{i 1}$ and $B_{i 2}$, with the segment between the balls deleted. By general position, we can assume that $L$ is disjoint from each $\ell_{i}$. It is now easy to construct a contact isotopy of $\mathbb{R}^{3}$ (for example, via a planar projection preserving $\frac{\partial}{\partial z}$ ) that pushes $L$ away from each $\ell_{i}$ and then entirely into the box, i.e., into standard form. Note that it is crucial here that $L$ not run in front of or behind $U_{i j}$. (Consider the difficulties inherent in Figure 10.)

To prove the final assertion of the theorem, suppose that $\left\{L_{t} \mid 0 \leq t \leq 1\right\}$ is an isotopy of Legendrian links in $\partial H$, with $L_{0}$ and $L_{1}$ in standard form. We wish to show that $L_{0}$ and $L_{1}$ are related by Moves 1-6. Let $T \subset(0,1)$ be the set of parameter values $t$ for which $L_{t}$ intersects some Legendrian curve $A_{i}$ or $\ell_{i}$. The homogeneity of contact structures allows us to construct enough deformations of a Legendrian isotopy to apply transversality theory, so we may assume that $T$ is finite, for $t \in T, L_{t} \cap \bigcup_{i}\left(A_{i} \cup \ell_{i}\right)$ is a single point, and near each such intersection the isotopy has a canonical form. After an additional 

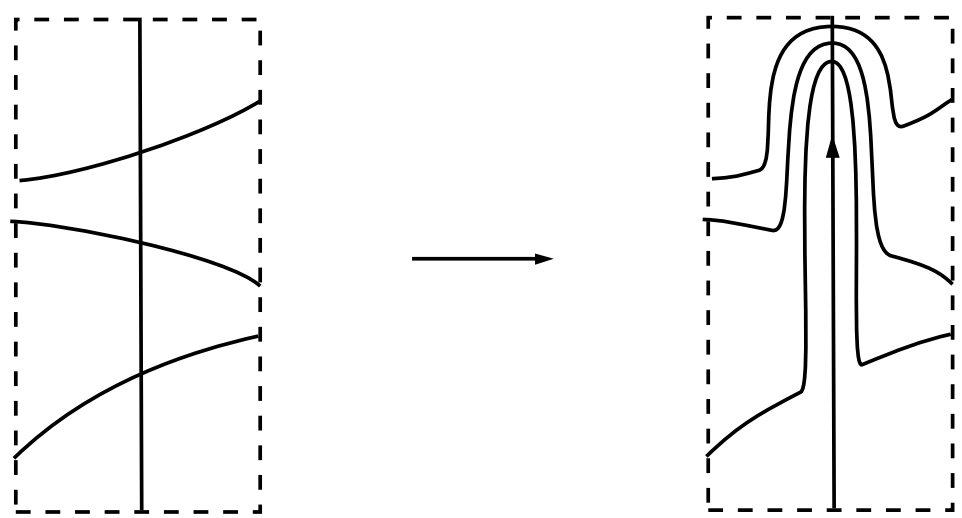

FIGURE 12

modification, we obtain a small $\varepsilon$ such that for each $t \in T$, the links with parameter between $t \pm \varepsilon$ are identical except for a canonical push across some $A_{i}$ or $\ell_{i}$. The complement of an $\varepsilon$-neighborhood of $T$ in $[0,1]$ is a finite collection of intervals $\left[t_{1}, t_{2}\right]$, each of which parametizes a Legendrian isotopy from $L_{t_{1}}$ to $L_{t_{2}}$ in $\partial H$ that is disjoint from each $A_{i}$ and $\ell_{i}$. The procedure of the previous paragraph simultaneously pushes each isotopy into the box, after we suitably enlarge $T$ to allow for intersections with $\ell_{i}$ created by the first isotopy $F_{s}$. (Note that while we previously disallowed links projecting onto any $p_{i j}$, a transverse pass across $p_{i j}$ does not affect the construction.) Each $L_{t}$ is sent to a Legendrian link $L_{t}^{\prime}$ in the box, and we can assume that the endpoints $L_{t_{k}}^{\prime}$ of each isotopy are in standard form. Now if we identify opposite edges of the box at the 1-handles, we essentially have an ordinary front projection of isotopies of Legendrian links, together with a set of distinguished vertical arcs where we have glued. Thus, we can reduce each isotopy between $L_{t_{1}}^{\prime}$ and $L_{t_{2}}^{\prime}$ to a sequence of Moves 1-3 (cf. [S]), together with Moves 4 and 5 to account for sliding cusps and double points over 1-handles. We will complete the proof by showing that for each $t \in T$, the links $L_{t-\varepsilon}^{\prime}$ and $L_{t+\varepsilon}^{\prime}$ are related by Moves 1-6, as are the links $L_{t}$ and $L_{t}^{\prime}$ for $t=0,1$.

Suppose that $t \in T$ corresponds to an intersection with some $\ell_{i}$. At the intersection, we can take a local model of the front projection of $L_{t}$ (or $\left.F_{1}\left(L_{t}\right)\right)$ to be a parabola tangent to $\ell_{i}$, and the isotopy from $L_{t-\varepsilon}$ to $L_{t+\varepsilon}$ to correspond to a vertical translation. Tracing through the above procedure for producing $L_{t \pm \varepsilon}^{\prime}$, we see that these links will differ by Move $6^{\prime}$ of Figure 13 . Figure 14 shows how to reduce Move $6^{\prime}$ to Moves 1-6. The same argument, with the isotopy $F_{s}$ of the previous paragraph taken to be the identity, shows that two links in standard form are related by Moves 1-6 if they are related by a Legendrian isotopy in $\partial H$ disjoint from each $S_{i j}-U_{i j}$. It follows that $L_{t}$ and $L_{t}^{\prime}$ are so related for $t=0,1$.
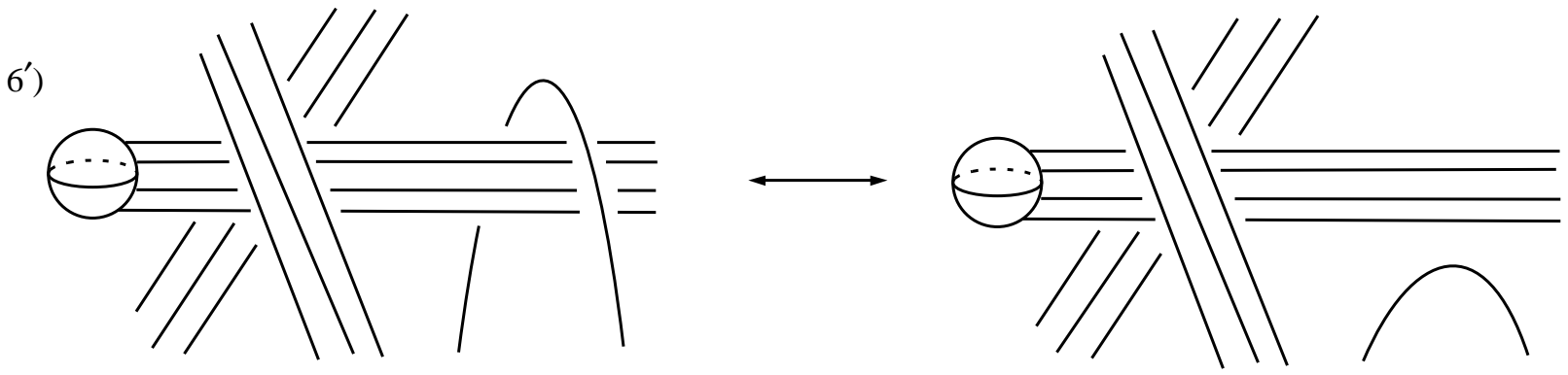

FIGURE 13

For the remaining values $t \in T$, we have $L_{t}$ intersecting some $A_{i}$. Now we must analyze the effect of the isotopy $F_{s}$ of Figure 12 on pictures in standard form. Recall that the top and bottom edges of Figure 12 are identified, and that $A_{i}$ projects to the identified endpoints of the vertical arc representing 

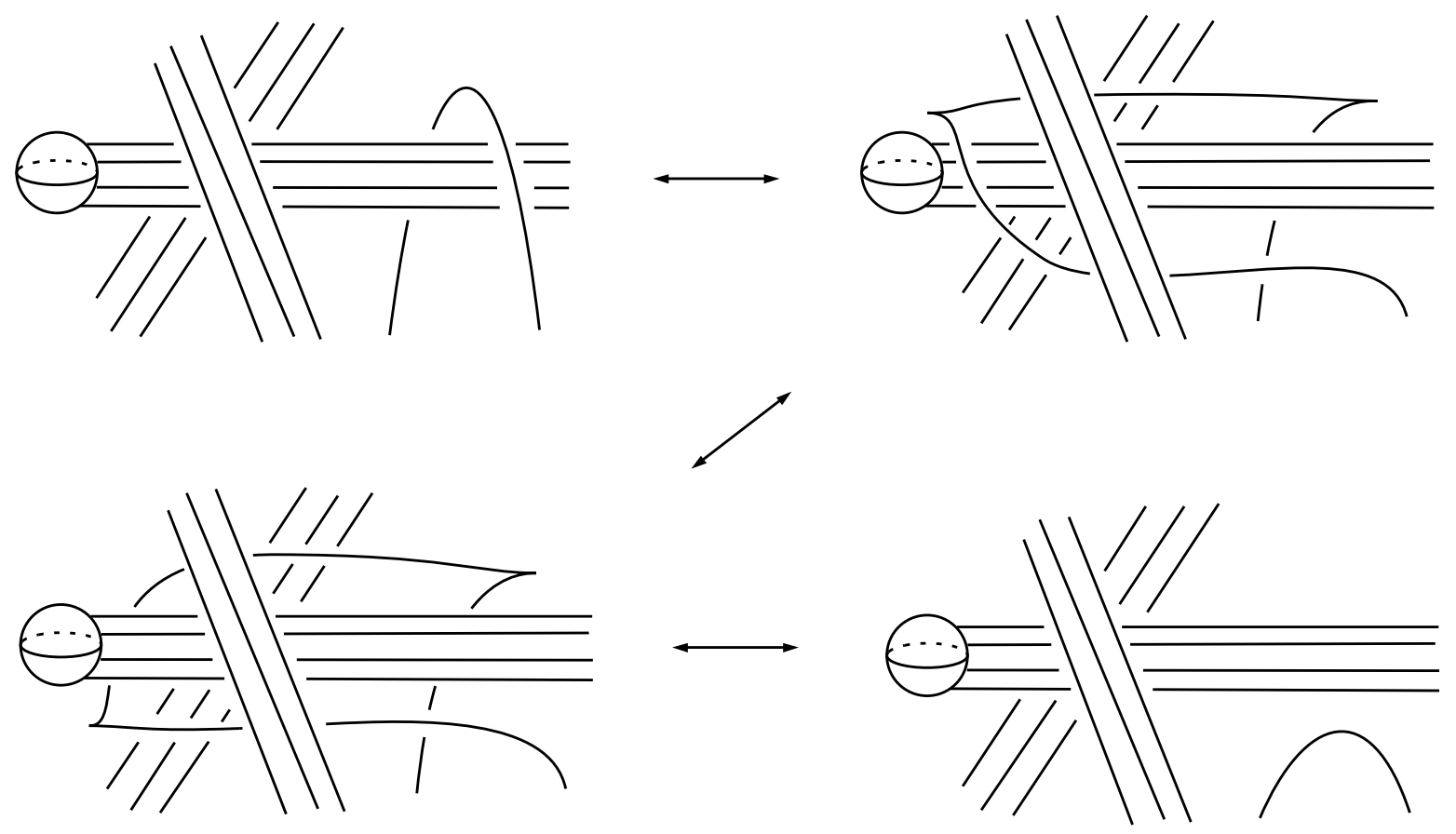

FIGURE 14

$S_{i}$. Allowing isotopies of $L_{t \pm \varepsilon}^{\prime}$ disjoint from $S_{i j}-U_{i j}$, and reversing the sign of $\varepsilon$ if necessary, we can assume that $L_{t-\varepsilon}$ is given near $L_{t} \cap A_{i}$ by a horizontal arc through a point $p_{-}$in $S_{i}$ near the top edge of Figure 12, and that this curve is fixed by $F_{s}$. Similarly, we can obtain $L_{t+\varepsilon}$ from $L_{t-\varepsilon}$ by pushing the point of intersection upward through $A_{i}$, to a point $p_{+}$near the bottom of the figure. Thus the isotopy $F_{s}$ will fix $p_{-}$and move $p_{+}$to the top of the picture. We can assume that the maps from a neighborhood of $L_{t} \cap A_{i}$ in Figure 12 to $U_{i 1}$ and $U_{i 2}$ in Figure 8 are sufficiently well approximated by translations in $\mathbb{R}^{3}$ that $F_{1}$ aligns the points of $L_{t \pm \varepsilon} \cap S_{i j}$ vertically in $S_{i 1}$ and $S_{i 2}$, with $F_{1}\left(p_{+}\right)$on the bottom and $p_{-}$ on top. To translate Figure 12 into a standard picture of $\partial H$, we first draw the $z$-axis of Figure 12 as a transverse equatorial circle in each $S_{i j}$, projecting to a figure-8. (See Figure 15.) The arrows indicate the direction of $F_{s}\left(\frac{\partial}{\partial z}\right.$ in Figure 12). Note that this orients the equators of both spheres counterclockwise, and is consistent with an orientation-reversing gluing map $\varphi_{i}: S_{i 1} \rightarrow S_{i 2}$ interchanging the poles. (We can assume that $\varphi_{i}$ identifies the equators by $180^{\circ}$ rotation about the $z$-axis.) The isotopy $F_{s}$ will push $p_{+}$counterclockwise once around each equatorial curve. To determine its effect on $L_{t+\varepsilon}$, we examine the curves in Figure 12. On each segment where the second derivative has large magnitude, the curve will be traveling nearly parallel to the characteristic foliation of $S_{i}$ (a left-handed spiral in Figure 15). Where the first derivatives have large magnitude, the curves travel crosswise to the foliation near the poles of $S_{i}$. By checking orientations, we see that these parts of the curves will lie above both northern hemispheres in Figure 15. Figures 15 and 16 (and the image of Figure 16 under $180^{\circ}$ rotation about the $z$-axis) show the isotopy $F_{s}$. Completing our contact isotopy to obtain $L_{t \pm \varepsilon}^{\prime}$, we see that these two links differ by the move shown in Figure 17. (We can remove the extra spirals by Move 1. Note that the other curves in Figure 12 lie farther from $S_{i j}$ in Figures 15 and 16, as does the rest of $L_{t \pm \varepsilon}$, so these do not interfere, and can be pushed away toward the center of the box in Figure 17 by Moves 2 and 3.) Figure 18 provides the reduction from this move to Moves 1-6.

Remarks. It is easily checked that Move $6^{\prime}$ (Figure 13) is equivalent to Move 6 (Figure 9) in the presence of Moves 1-3 (Figure 3). Figure 18 generalizes to give a derivation of the move in Figure 19, which represents a collection of strands being looped around and under a 1-handle. Of course, this contact isotopy preserves the canonical framing of each knot $K$, as well as $r(K)$, but the integer $t b(K)$ changes 

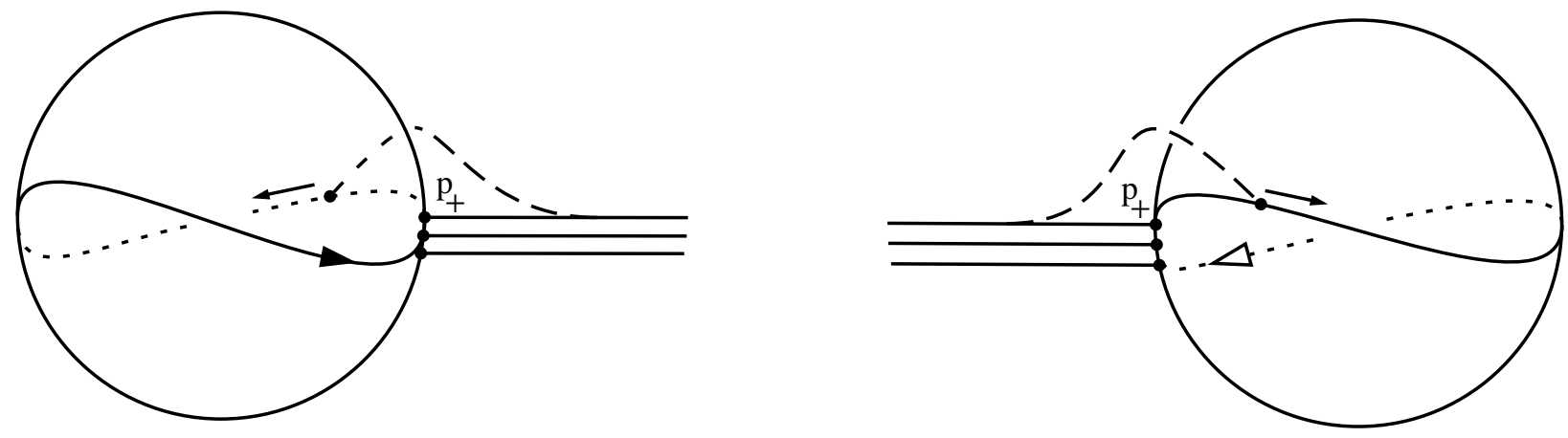

FigURE 15

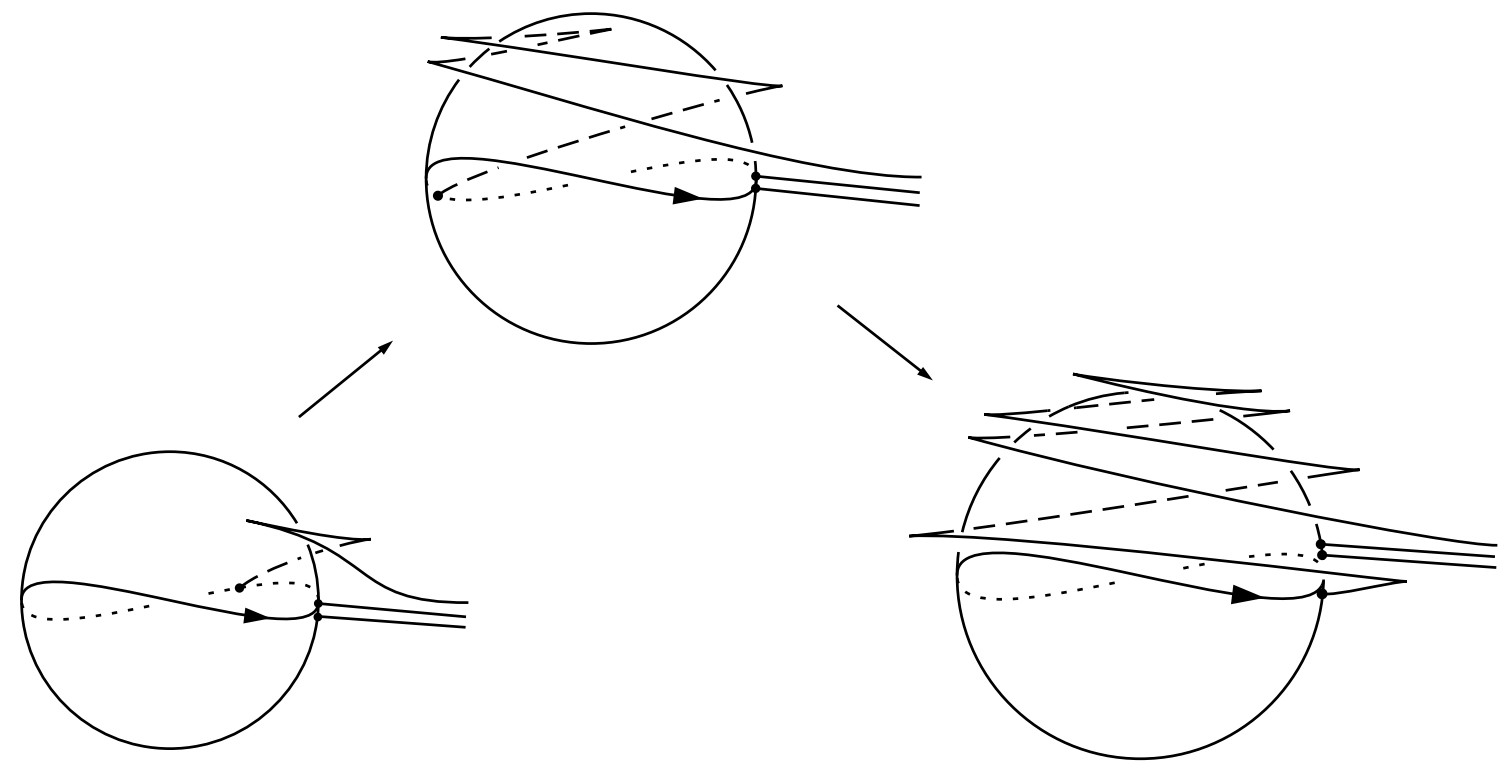

FiguRE 16
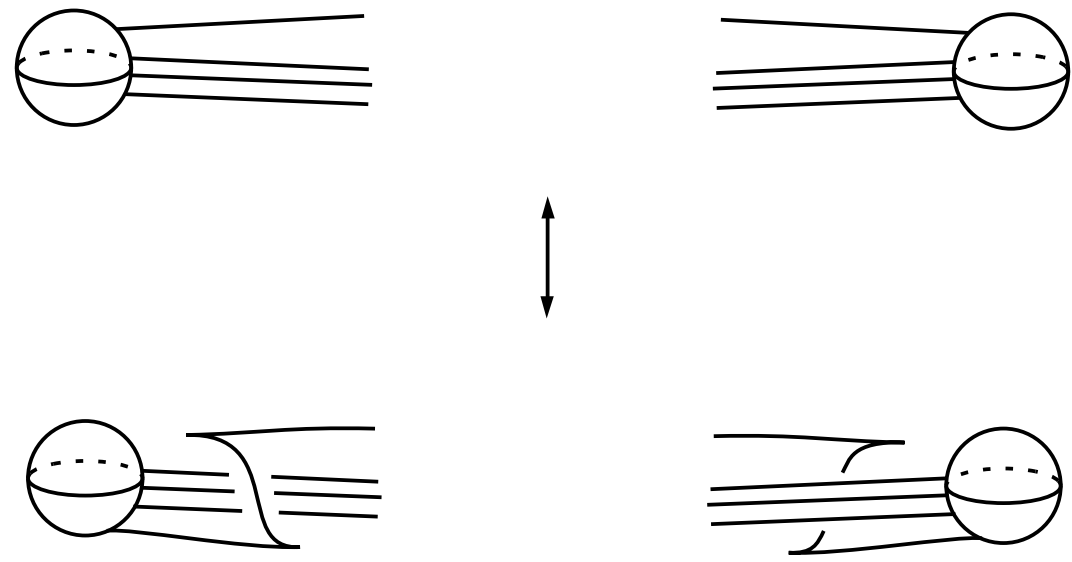

FiguRE 17

in general. A related example is given by Figure 20, which shows how we can convert a right crossing to 
a left one, by a smooth (not contact) isotopy that adds a right twist to the canonical framing of $K$. Such tricks can be useful for putting Stein structures on preassigned smooth 4-manifolds.
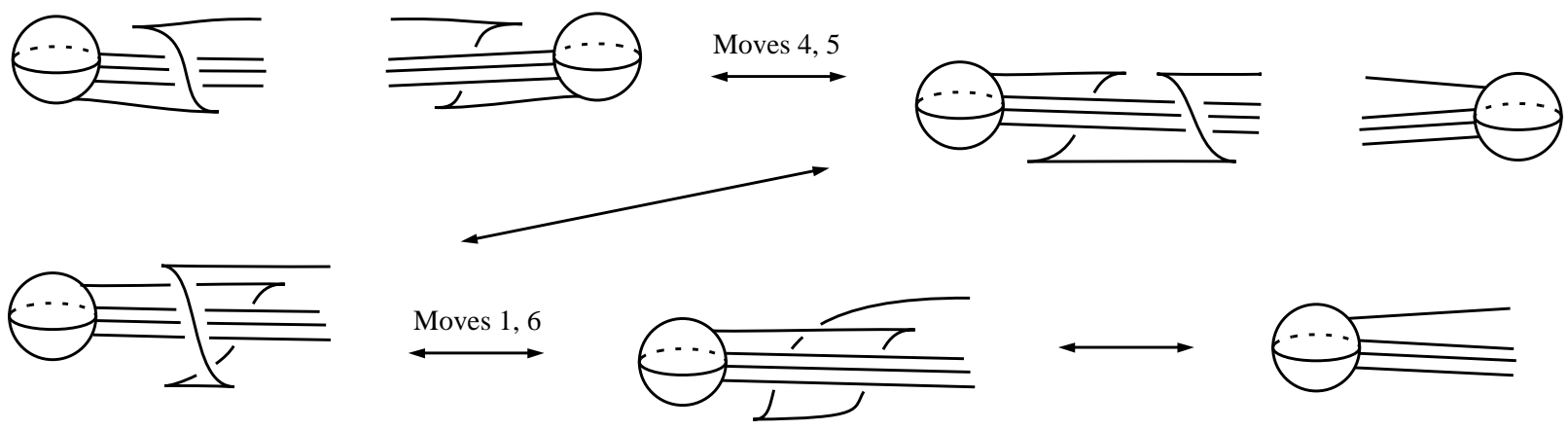

FiguRE 18
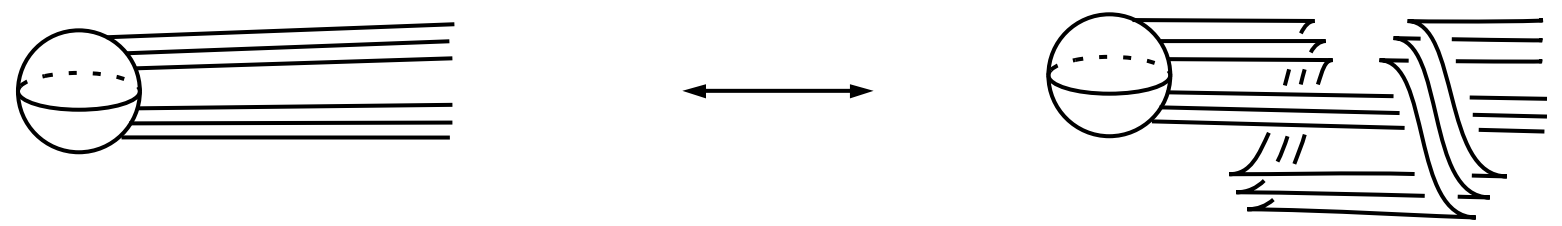

FiguRE 19

Implicit in the proof of Theorem 2.2 are nontrivial self-equivalences of $H$ (up to Stein-homotopy [E6]) that are smoothly isotopic to the identity. We found a contactomorphism $\psi_{i}$ that twisted the vector field in $\partial H$ corresponding to $\frac{\partial}{\partial x}$, by rotating $B_{i 1}$ through $360^{\circ}$. The proof of the theorem shows that $\psi_{i}$ acts on Legendrian links as in Figure 21, up to contact isotopy. (For example, start with horizontal arcs in Figure 12, then apply a vertical Dehn twist on one side.) Figure 19 verifies that these moves for $\psi_{i}$ and $\psi_{i}^{-1}$ are indeed inverses up to contact isotopy. In our alternate picture of $S^{1} \times S^{2}, \psi_{1}$ is given by a Dehn twist on the torus, $\psi_{1}(x, y, z)=(x-1, y, y+z)$ on $\mathbb{R}^{3} / 0 \times 2 \pi \mathbb{Z}^{2}$. Now $\psi_{i} \circ \psi_{i}$ is smoothly isotopic to the identity, since $\pi_{1}(S O(3)) \cong \mathbb{Z}_{2}$. However, no nonzero power of $\psi_{i}$ is contact isotopic to the identity. This is because $\psi_{i}$ twists any nonzero vector field $v$ in $\xi$, and $\pi_{1}(S O(2)) \cong \mathbb{Z}$. For example, if $K$ runs once over the 1-handle, then $\psi_{i}$ will increase the rotation number of $K$ by 1 , so $\psi_{i}^{n}(K)$ will not be contact isotopic to $K$ for $n \neq 0$. Clearly, we obtain other automorphisms of $H$, which are nontrivial on $\pi_{1}$, by changing the other auxiliary data. Changes of order or directions of the 1-handles correspond to various permutations of the balls $B_{i j}$. We can reverse the orientation of $\xi$ by rotating about the $x$ - or $y$-axis. Allowing other handle structures on $H$ increases the symmetries (by 1-handle slides).

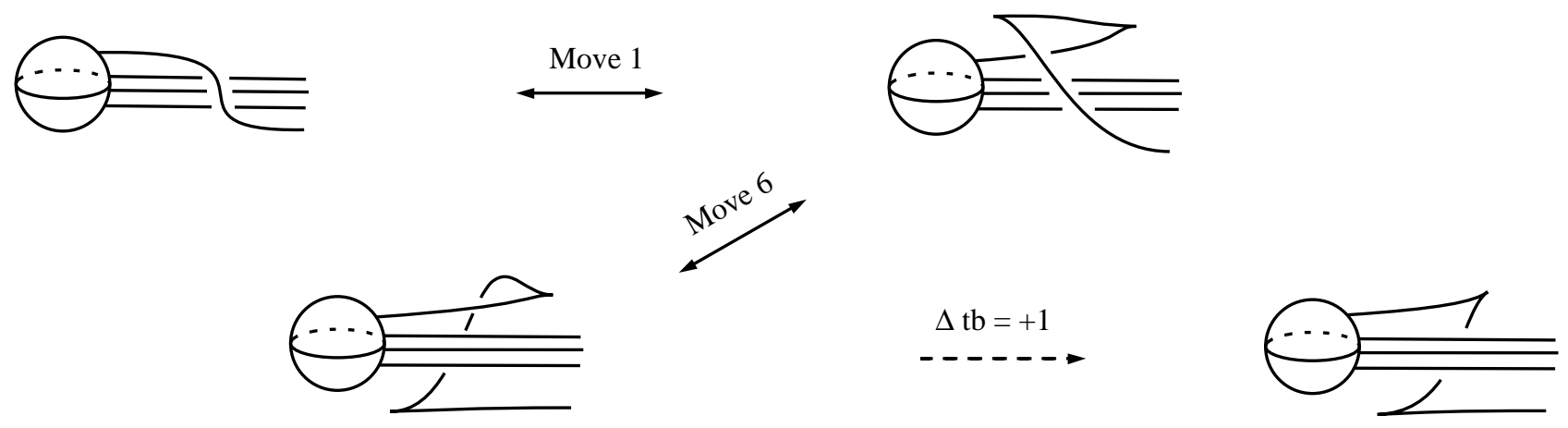

FiguRE 20 

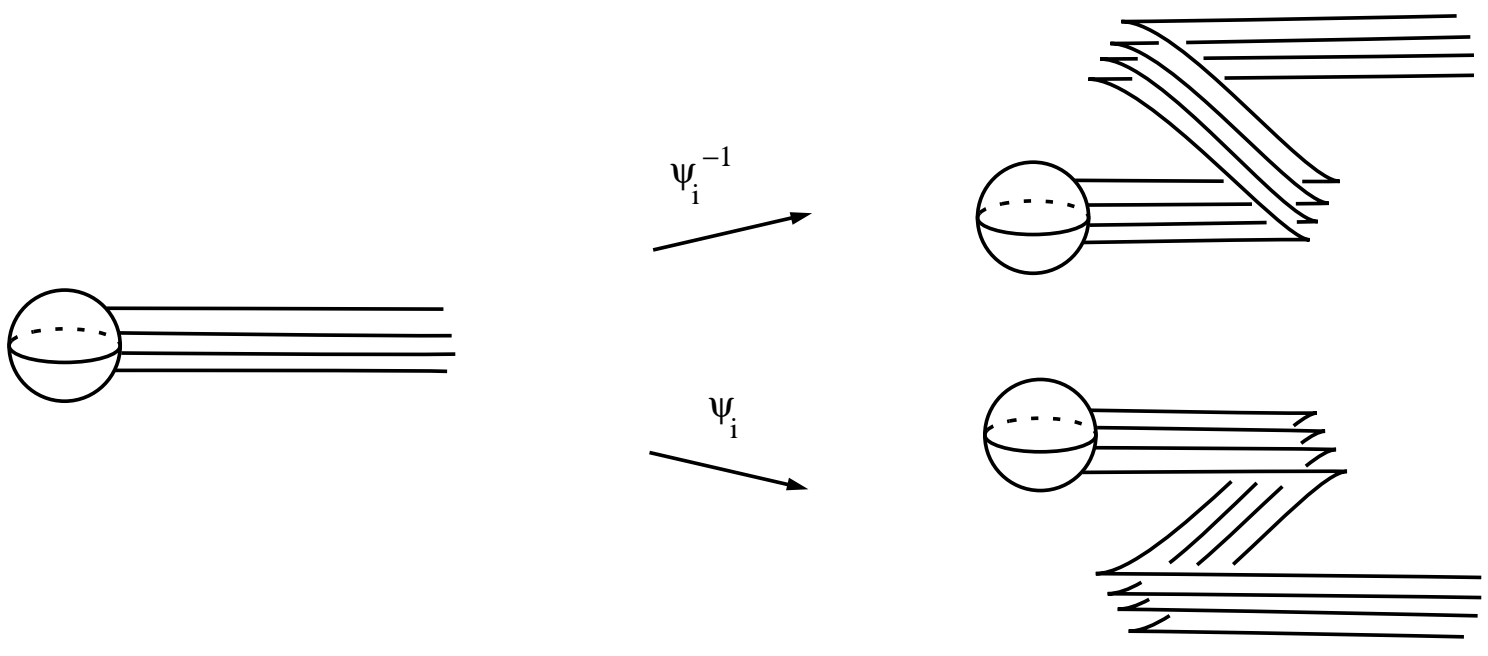

FiguRE 21

\section{Exotic Stein surfaces.}

In this section, we give a simple characterization of those manifolds that are homeomorphic to Stein surfaces. We obtain many new examples of Stein surfaces, most of which are in some sense "exotic" as smooth manifolds. For example, we obtain an uncountable family of nondiffeomorphic Stein surfaces, all of which are homeomorphic to $\mathbb{R}^{4}$ ("exotic" $\mathbb{R}^{4}$ 's).

Theorem 3.1. An open, oriented, topological 4-manifold $X$ is homeomorphic to a Stein surface if and only if it is the interior of a (possibly infinite) handlebody $H$ without handles of index $>2$. If so, then any almost-complex structure on $X$ (up to homotopy) is induced by an orientation-preserving homeomorphism from a Stein surface.

To clarify the last statement, we must specify the meaning of an almost-complex structure (up to homotopy) on the topological manifold $X$. Any 4-dimensional topological handlebody can be canonically smoothed, since the gluing maps are flat topological embeddings of 3-manifolds, and these are always uniquely smoothable. Thus, we can define almost-complex structures on $X$ in the usual way relative to a fixed smoothing. To ensure that homeomorphisms induce canonical correspondences of such structures, however, requires more work. Recall (e.g., $[\mathrm{KM}],[\mathrm{T}]$ ) that an almost-complex structure on a smooth 4-manifold determines a $\operatorname{spin}^{c}$-structure. In our case $H^{i}(X ; \mathbb{Z})=0$ for $i>2$, so the correspondence is bijective (since $\operatorname{Spin}^{c}(4) / U(2) \approx S^{3}$ is 2-connected), and it suffices to define spinc-structures on oriented topological 4-manifolds. Every such manifold has a topological tangent bundle with structure group STop(4), the group of orientation-preserving homeomorphisms of $\left(\mathbb{R}^{4}, 0\right)[\mathrm{KS}]$. Since the inclusion $S O(4) \hookrightarrow \operatorname{STop}(4)$ is double covered by an inclusion $\operatorname{Spin}(4) \hookrightarrow \operatorname{SpinTop}(4)$, we can replace $\operatorname{Spin}(4)$ by SpinTop(4) in $\operatorname{Spin}^{c}(4)=\left(S^{1} \times \operatorname{Spin}(4)\right) / \mathbb{Z}_{2}$, and construct a theory of topological $\operatorname{spin}^{c}$-structures that reduces to the usual theory in the presence of a smooth structure [G3]. Orientation-preserving homeomorphisms will preserve these structures in the same manner that diffeomorphisms do, so we obtain a homeomorphism-invariant notion of almost-complex structures on $X$. Absolute and relative Chern classes $c_{1}$ will be preserved by orientation-preserving homeomorphisms, since they are determined by the complex line bundles associated to the corresponding topological spin ${ }^{c}$-structures. (A related argument applies to homotopy equivalences [G3].)

To enumerate almost-complex structures on $X$, we smooth $X$ as int $H$. Since $S O(4) / U(2) \approx S^{2}$ is simply connected, almost-complex structures exist, and we can assume that they all agree on the union $X_{1}$ of 0 - and 1-handles. After fixing a complex trivialization $\tau$ there, we obtain a relative Chern class in $H^{2}\left(X, X_{1} ; \mathbb{Z}\right)$ for each almost-complex structure. These will reduce modulo 2 to the relative StiefelWhitney class $w_{2}(X, \tau) \in H^{2}\left(X, X_{1} ; \mathbb{Z}_{2}\right)$. Since $\pi_{2}(S O(4) / U(2)) \cong \mathbb{Z}$, obstruction theory produces a surjection from integer lifts of $w_{2}(X, \tau)$ to almost-complex structures, via $c_{1}$. (The map fails to be injective, since there will be nontrivial self-homotopies of the structure over $X_{1}$, changing $\tau$ by any even 
number of twists. In fact, the set of almost-complex structures on $X$ is affinely $H^{2}(X ; \mathbb{Z})$, with difference cocycles given by half the difference in relative Chern classes.)

Proof. Clearly, any manifold homeomorphic to a Stein surface has the required handle structure. (See Theorem 1.3.) For the converse, we start with $X=\operatorname{int} H$ as in the theorem, and construct a Stein surface homeomorphic to $X$. We assume (as above) that $H$ is a smooth handlebody, and fix a trivialization $\tau$ of the unique (up to homotopy) almost-complex structure on $X_{1}$, the intersection of $X$ with the 0 - and 1-handles of $H$. We pick an integer lift $c \in H^{2}\left(X, X_{1} ; \mathbb{Z}\right)$ of $w_{2}(X, \tau)$, and arrange for the homeomorphism to map $c$ onto the Chern class of the Stein surface (relative to the corresponding trivialization on the preimage of $X_{1}$ ). The theorem then follows from the discussion above.

Consider a handlebody $H^{\prime}$ obtained from $H$ by removing each 2-handle $h_{i}$ and regluing it along the same attaching circle, but with an even number $2 k_{i}$ of left (negative) twists added to its framing. By Eliashberg's Theorem (1.3), int $H^{\prime}$ will be Stein if each $k_{i}$ is sufficiently large. Clearly, $H^{\prime}$ is canonically homotopy equivalent rel $X_{1}$ to $H$ and $X$, with $w_{2}\left(H^{\prime}, \tau\right)$ mapping to $w_{2}(X, \tau)$ since the numbers of twists were even. Thus, $c_{1}\left(H^{\prime}, \tau\right)$ and $c$ differ by an even class in $H^{2}\left(X, X_{1} ; \mathbb{Z}\right)$. Since adding 2 left twists to the framing of $h_{i}$ allows us to change $c_{1}\left(H^{\prime}, \tau\right)$ by \pm 2 on $h_{i}$ (cf. Figure 7 and Proposition 2.3), we can assume (for each $k_{i}$ sufficiently large) that $c_{1}\left(H^{\prime}, \tau\right)$ maps to $c$ under the homotopy equivalence.

Our homotopy equivalence between $H^{\prime}$ and $X$ does not preserve the intersection pairing, a defect which we will remedy at the expense of increasing $\pi_{1}$, as follows. Close inspection of Eliashberg's paper [E2] shows that we can put self-plumbings of either sign in each of the 2-handles of $H^{\prime}$, without changing the gluing maps, and after smoothing, still have a manifold $H^{\prime \prime}$ whose interior is Stein. (A self-plumbing is performed by choosing a pair of disjoint disks $D, D^{\prime} \subset D^{2}$ and gluing $D \times D^{2}$ to $D^{\prime} \times D^{2}$ in the 2-handle $D^{2} \times D^{2}$, by a diffeomorphism interchanging the factors. Since the 2-handles are constructed by Lemmas 3.5.1 and 3.4.3 of [E2], we can assume that part of each handle is given as an arbitrarily narrow $\varepsilon$-neighborhood of $D^{2} \times 0$ in $i \mathbb{R}^{2} \times \mathbb{R}^{2}$. We can then do the self-plumbings, smoothing by Lemma 3.4.4 to preserve the Stein property.) Alternatively, we may do each plumbing explicitly in a link picture by adding a 1-handle as in Figure 22 (ignoring the dashed curves) (cf. [C], [GS]). Suppose we obtain $H^{\prime \prime}$ from $H^{\prime}$ by adding $k_{i}$ self-plumbings (counted with sign) to each $h_{i}$, transforming it into a kinky handle $h_{i}^{\prime \prime}$. There is a canonical local diffeomorphism $\varphi: H^{\prime} \rightarrow H^{\prime \prime}$ rel $X_{1}$ that induces isomorphisms on $H_{2}$ and $H^{2}$. Since $\varphi$ preserves the almost-complex structures, the Chern class $c_{1}\left(H^{\prime \prime}, \tau\right)$ corresponds to $c \in H^{2}\left(X, X_{1} ; \mathbb{Z}\right)$ under the obvious isomorphism. Furthermore, the given isomorphism $H_{2}\left(H^{\prime \prime} ; \mathbb{Z}\right) \cong H_{2}(X ; \mathbb{Z})$ preserves the intersection pairing. In fact, by the most natural way of defining the attachment of kinky handles along framed circles, the handles $h_{i}^{\prime \prime}$ are actually attached along the framed curves defining the original handlebody $H[\mathrm{C}]$. To understand this, recall that for closed surfaces generically immersed in 4-manifolds, the homological intersection number differs from the normal Euler number by twice the signed number of self-intersections. Thus, the above convention for attaching kinky handles, correcting the framing by twice the signed number of self-plumbings, guarantees that the resulting intersection pairing only depends on the framed link, and not on the numbers of self-plumbings of the kinky handles. Alternatively, observe that if we add self-plumbings to $h_{i}$ using Figure 22, each \pm self-plumbing will increase the ThurstonBennequin invariant of the attaching circle by \pm 2 and leave its rotation number unchanged, as required.

Finally, we eliminate the unwanted extra $\pi_{1}$ by extending the kinky handles $h_{i}^{\prime \prime}$ to Casson handles. According to Casson $[\mathrm{C}]$, each kinky handle $h_{i}^{\prime \prime}$ has a canonical framed link in its boundary, such that if we add 2-handles along the framed link, $h_{i}^{\prime \prime}$ will be transformed into a standard 2-handle, and the above-mentioned natural framing on the attaching circle of $h_{i}^{\prime \prime}$ will correspond to the product framing on the 2-handle. Thus, attaching 2-handles to all kinky handles $h_{i}^{\prime \prime}$ in this manner would transform $H^{\prime \prime}$ back into $H$. In general, we cannot do this in the Stein setting, but our previous argument shows that we can add kinky handles to these framed links to obtain a new manifold whose interior is Stein. (In fact, the relevant circles appear in Figure 22 as dashed curves with the zero framing, so we can add any kinky handles with more positive than negative self-plumbings.) We iterate the construction, adding a third layer of kinky handles onto the second layer, and continue, to construct a manifold $\widehat{H}$ with infinitely many layers of kinky handles. Clearly, int $\widehat{H}$ is Stein. But for each infinite stack of kinky handles (starting with some $h_{i}^{\prime \prime}$ ), the interior union the attaching region is (by definition) a Casson handle [C]. Freedman $[\mathrm{F}]$ proved that any Casson handle is homeomorphic to an open 2-handle $D^{2} \times \mathbb{R}^{2}$ such that the natural 

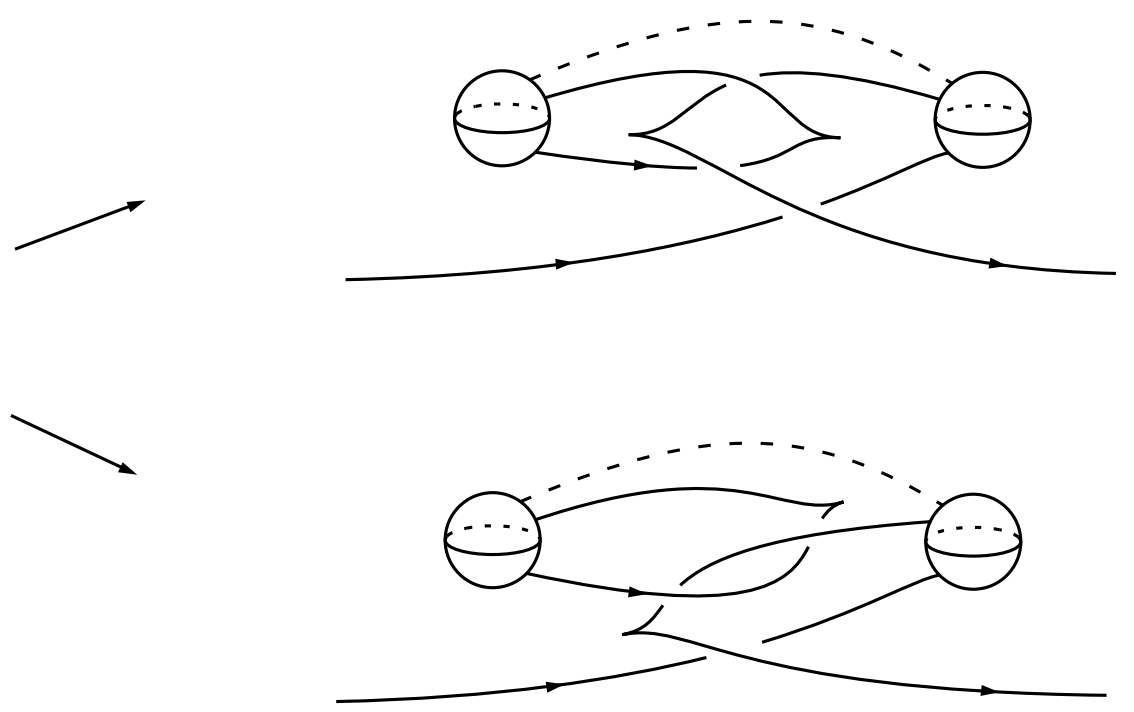

$(-)$

FiguRE 22

framings of the attaching circles correspond. Thus, the Stein surface int $\widehat{H}$ is homeomorphic rel $X_{1}$ to int $H=X$. The restriction map $H^{2}\left(\widehat{H}, X_{1} ; \mathbb{Z}\right) \rightarrow H^{2}\left(H^{\prime \prime}, X_{1} ; \mathbb{Z}\right)$ is an isomorphism preserving $c_{1}$, so the given homeomorphism int $\widehat{H} \approx X$ maps $c_{1}(\widehat{H}, \tau)$ onto $c$ as required.

A related observation applies in the compact setting. If $H$ is a 4-dimensional compact handlebody without handles of index $>2, X_{1}$ denotes its 1 -skeleton, and $c \in H^{2}\left(H, X_{1} ; \mathbb{Z}\right)$ and $\tau$ on $X_{1}$ specify an almost-complex structure on $H$, then there is a compact Stein surface $X$ with boundary, and a homotopy equivalence $\varphi: X \rightarrow H$ rel $X_{1}$ preserving the intersection pairing and with $\varphi^{*}(c)=c_{1}(X, \tau)$. To see this, simply note that for a Legendrian knot $K, t b(K)$ can be increased by any even number without changing $r(K)$, by forming the connected sum with a knot with sufficiently large $t b$ and $r=0$. For example, we can realize any finitely presented group as $\pi_{1}(X)$ or any finite rank, symmetric $\mathbb{Z}$-bilinear form as the intersection pairing of such a compact $X$.

Corollary 3.2. Any smooth, closed, connected, oriented 4-manifold contains a smooth, finite wedge of circles whose complement is homeomorphic to a Stein surface. Any smooth (resp. topological) open, oriented 4-manifold contains a smooth (resp. locally flat) 1-complex whose complement is homeomorphic to a Stein surface.

Proof. In the topological case, the manifold admits a smooth structure [Q], [FQ]. Now in either case, there is a handle decomposition, and the required 1-complex is dual to the 3- and 4-handles.

Since the Stein surfaces constructed in the proof of Theorem 3.1 are built with Casson handles, the underlying smooth manifolds will typically be "exotic" in some sense. It seems likely that any $X$ will admit uncountably many diffeomorphism types of such homeomorphic Stein surfaces, and that no such smooth manifold can admit a proper Morse function with finitely many critical points (provided that $X$ is not homeomorphic to the interior of $B^{4} \cup 1$-handles). We illustrate this behavior with a concrete example.

Theorem 3.3. For each integer $n$, let $H_{n}$ denote the compact 2-disk bundle over $S^{2}$ with Euler number $n$. Then int $H_{n}$ is (orientation-preserving) homeomorphic to a Stein surface $V_{n}$ that contains no smoothly embedded sphere generating its homology and realizes any preassigned almost-complex structure. For $n= \pm 1$, there are uncountably many diffeomorphism types of such manifolds $V_{ \pm 1}$, none of which admit proper Morse functions with finitely many critical points.

In contrast, the manifolds $\partial H_{0}=S^{1} \times S^{2}, \partial H_{ \pm 1}=S^{3}$ and $\partial H_{ \pm 2}=\mathbb{R} P^{3}$ admit unique tight contact structures, and these are uniquely fillable (up to blowing up) by $S^{1} \times B^{3}, B^{4}$ and $H_{-2}$, respectively (by 
work of Gromov [Gro] and Eliashberg, see [E3]). Thus, $H_{0}=S^{2} \times D^{2}, H_{1}=\mathbb{C} P^{2}-\operatorname{int} B^{4}$ and $H_{2}$ are not diffeomorphic to Stein surfaces with boundary (or even symplectic manifolds with convex boundaries), although their interiors admit exotic smooth structures that are Stein. Clearly, we could construct many other examples of manifolds with these boundaries, whose interiors are homeomorphic to Stein surfaces. (Consider closed manifolds minus int $B^{4}$, int $S^{1} \times B^{3}$ or int $H_{ \pm 2}$.)

Proof. The manifold $H_{n}$ is obtained from $B^{4}$ by gluing a 2-handle to an unknot in $\partial B^{4}$ with framing $n$. For any Casson handle $\mathrm{CH}$, let $V_{n}(\mathrm{CH})$ be the interior of the manifold obtained by gluing $\mathrm{CH}$ to $B^{4}$ along an $n$-framed unknot. Then by the proof of Theorem 3.1, $V_{n}(C H)$ will be realized as a Stein surface homeomorphic to int $H_{n}$ and realizing the preassigned almost-complex structure, provided that $C H$ has a suitable excess of positive self-plumbings.

There are many known examples of smooth, simply connected 4-manifolds $M$ whose intersection pairing contains a subspace with pairing

$$
\left[\begin{array}{cc}
n & 1 \\
1 & \text { even }
\end{array}\right]
$$

such that the class $\alpha$ with square $n$ cannot be represented by a smoothly embedded sphere. (For $n \geq 0$ see, for example, $[\mathrm{FM}]$ Chapter 6, Corollary 4.2. For $n<0$, simply reverse orientation.) By Casson's Embedding Theorem [C], however, we can find a Casson handle $C H$ such that $V_{n}(\mathrm{CH})$ embeds in $M$ representing $\alpha$. We can always add self-plumbings and layers of kinky handles to $C H$, so that it becomes suitably positive and $V_{n}(\mathrm{CH})$ admits a Stein structure as above. It cannot contain a smooth sphere generating its homology, since this would also represent $\alpha$ in $M$.

Now suppose $n=1$. Then we may choose $M$ so that the orthogonal complement of $\alpha$ in the intersection pairing is nonstandard and negative definite [G1]. As in Freedman [F], we can construct a nested family $\left\{\mathrm{CH}_{c}\right\}$ of Casson handles inside $\mathrm{CH}$, indexed by a Cantor set. At each stage of the construction, we add positive self-plumbings wherever necessary so that each $V_{1}\left(C H_{c}\right)$ admits a Stein structure as above. If any two of the nested manifolds $V_{1}\left(\mathrm{CH}_{c}\right)$ in $M$ were diffeomorphic, then a standard argument [G2] would allow us to contradict the Periodic End Theorem of Taubes. If any one had a proper Morse function with finitely many critical points, then its end would be smoothly collared by a 3-manifold crossed with $\mathbb{R}$, and the same argument would apply. For $n=-1$, the same argument works in the manifold $\bar{M}$.

We reformulate our result about gluing Casson handles in the context of Legendrian link presentations of Stein surfaces (Section 2). A kinky handle with $k_{+}$positive and $k_{-}$negative self-plumbings can be attached along a Legendrian knot $K$ in $\# n S^{1} \times S^{2}$ with framing $m$ (after allowing a $C^{0}$-small perturbation of $K$ to lower $t b$ if necessary), provided that $m \leq t b(K)-1+2\left(k_{+}-k_{-}\right)$. This kinky handle can be extended to a Casson handle, and the only restriction on this extension is that for each of the additional kinky handles we must have $k_{+}>k_{-}$. The rotation number $r(K)$ (after the perturbation of $K$ to achieve equality in the above formula) contributes to the Chern class as if the Casson handle were an ordinary 2-handle. We now obtain the following theorem, that some exotic $\mathbb{R}^{4}$ 's admit Stein structures.

Theorem 3.4. There are uncountably many diffeomorphism types of Stein surfaces homeomorphic to $\mathbb{R}^{4}$. There is a Stein exotic $\mathbb{R}^{4}$ that can be built with two 1-handles, one 2-handle and a single Casson handle $\mathrm{CH}$ with only one kinky handle at each stage (Figure 25).

Proof. In [BG], Bižaca and the author exhibit a particularly simple exotic $\mathbb{R}^{4}$. This is the interior $R$ of the manifold shown in Figure 23, which is essentially Figure 1 of [BG]. The circles with dots represent 1-handles (cf. $[\mathrm{K}],[\mathrm{GS}]$ ), the solid curve represents a 2-handle, and the dashed curve is where we attach the Casson handle $\mathrm{CH}$ with a single, positive self-plumbing in each kinky handle. Both framings are 0, as indicated. It is routine to verify that Figure 24 represents the same manifold $R$, where we are now attaching the handle and Casson handle to a 0-framed Legendrian link. (Change Figure 24 in the obvious way to represent the 1-handles by circles with dots. Then isotope to Figure 23.) Since the dashed curve has $t b=0$, we can attach the Casson handle $C H$ with framing 0 as required. However, the solid curve has $t b=-2$, so we must increase its canonical framing by 3 units by a smooth isotopy. This is easily accomplished by passing two strands around 1-handles (variations of the trick in Figure 20), resulting in Figure 25 of $R$, a Stein manifold homeomorphic but not diffeomorphic to $\mathbb{R}^{4}$. In [BG] it was also observed 


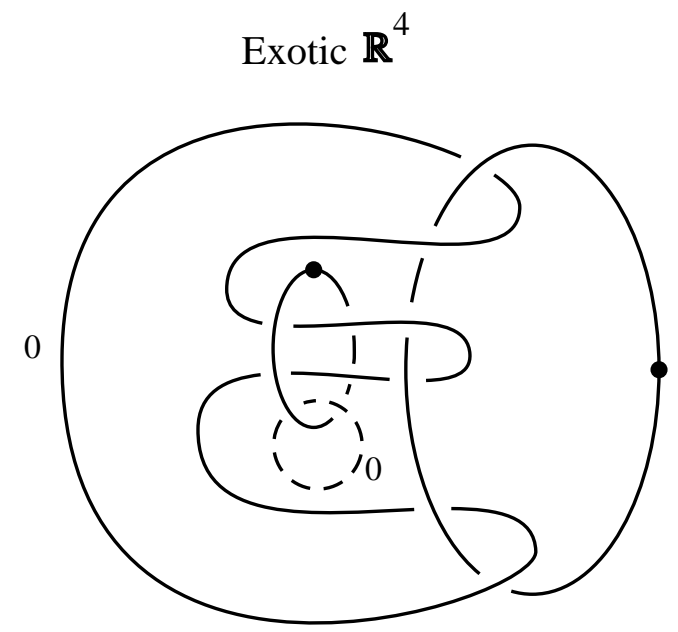

FiguRE 23

(as in [DF]) that $R$ contains an uncountable family of exotic $\mathbb{R}^{4}$ 's $\left\{R_{c}\right\}$ indexed by a Cantor set, produced using a nested family of Casson handles $\left\{\mathrm{CH}_{c}\right\}$ in $\mathrm{CH}$ as in the proof of Theorem 3.3. As before, we can arrange the family $\left\{\mathrm{CH}_{c}\right\}$ so that the manifolds $R_{c}$ (given by Figure 25 with $\mathrm{CH}_{c}$ in place of $\mathrm{CH}$ ) are all Stein. As in $[\mathrm{DF}]$, the family $\left\{R_{c}\right\}$ represents uncountably many diffeomorphism types.

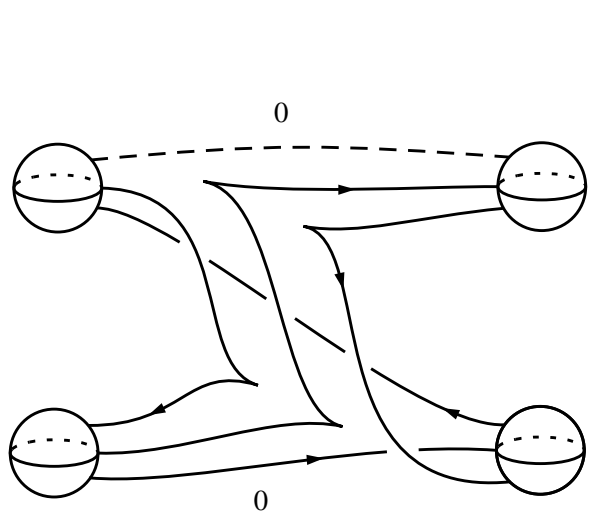

FiguRe 24
Stein exotic $\mathbb{R}^{4}$

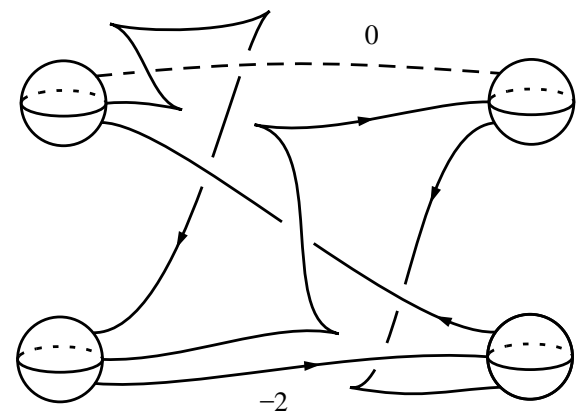

FiguRE 25

The Stein exotic $\mathbb{R}^{4}$ 's described here can all be smoothly embedded in the standard $\mathbb{R}^{4}$. There is another type of exotic $\mathbb{R}^{4}$ that cannot be so embedded [G2]. It is still an open (and apparently difficult) question whether any of these larger exotic $\mathbb{R}^{4}$ 's admit Stein structures.

\section{Invariants of 2-plane fields on 3-manifolds.}

In this section, we define a complete set of invariants for distinguishing homotopy classes of oriented 2-plane fields (or equivalently, nowhere zero vector fields or "combings") on oriented 3-manifolds. We show how to compute the invariants for the boundary of a compact Stein surface presented in standard form as in Section 2. We obtain various corollaries, including invariance of the rotation number (up to sign) and Thurston-Bennequin invariant for contact 3-manifolds obtained by surgery on Legendrian knots (Corollary 4.6).

At first glance, the classification of oriented 2-plane fields $\xi$ on an oriented 3-manifold $M$ seems to be easy with modern techniques. If we fix a trivialization of the tangent bundle $T M$, the problem becomes equivalent to classifying maps $\varphi: M \rightarrow S^{2}$ up to homotopy, and this latter problem was solved by Pontrjagin around $1940[\mathrm{P}]$. The difficulty is that the invariants depend on the choice of trivialization 
of $T M$. In fact, one obtains both a 2-dimensional obstruction (which is not necessarily determined by the Chern class $\left.c_{1}(\xi)\right)$ and a 3 -dimensional obstruction. However, if we classify only up to isomorphisms of $T M$, the problem reduces to classifying abstract, stably trivial 2-plane bundles on $M$, and this is accomplished by the Chern class. Thus, if we allow the trivialization of $T M$ to vary, all of our invariants except for the Chern class will be lost. The main problem, then, is how to construct invariants that capture the information contained in the homotopy class of $\xi$, but can be manipulated without the awkward task of keeping track of a trivialization of $T M$. We solve this problem by expressing the 3dimensional invariant in terms of a 4-manifold bounded by $M$, and the 2 -dimensional invariant in terms of a spin structure. Our strategy is first to understand the invariants relative to a fixed trivialization (Proposition 4.1). Then we discuss invariants $\Theta$ and $\theta$ that capture the 3-dimensional obstruction up to (at most) a $2: 1$ ambiguity (4.2-4.6 and preceding text). To resolve the ambiguity, we must understand the 2-dimensional obstruction $\Gamma$, which we analyze in 4.7-4.14. We give an explicit formula for $\Gamma$ that applies to the contact boundary of any compact Stein surface in standard form (Theorem 4.12) and give several applications. Finally, we exhibit the full 3-dimensional obstruction $\widetilde{\Theta}$, with further properties of $\theta$ and a sample of applications (4.15-4.20). We show that for Stein boundaries, the invariants $\Gamma$ and $\widetilde{\Theta}$ (or $\theta$ ) are independent from each other and from $c_{1}$, by exhibiting holomorphically fillable contact structures with $c_{1}=0$ on a fixed 3-manifold, and showing that they are distinguished by $\theta$ but not $\Gamma$ (Corollary 4.6) or vice versa (Example 4.14).

The first step in constructing the invariants is to understand the classification for a fixed trivialization of $T M$. For our purposes, it is convenient to use differential topology rather than Pontrjagin's original obstruction-theoretic method of proof $[\mathrm{P}]$. (See also Kuperberg $[\mathrm{Ku}]$ for a modern treatment via homotopy theory.) For an oriented 2-plane field $\xi$, let $d(\xi) \in \mathbb{Z}$ denote the divisibility of the Chern class, so that $c_{1}(\xi)$ equals $d(\xi)$ times a primitive class in $H^{2}(M ; \mathbb{Z})$ modulo torsion, and $d(\xi)=0$ if $c_{1}(\xi)$ is of finite order.

Proposition 4.1. Let $M$ be a closed, connected 3-manifold. Then any trivialization $\tau$ of the tangent bundle of $M$ determines a function $\Gamma_{\tau}$ sending homotopy classes of oriented 2-plane fields $\xi$ on $M$ into $H_{1}(M ; \mathbb{Z})$, and for any $\xi, 2 \Gamma_{\tau}(\xi)$ is Poincaré dual to $c_{1}(\xi) \in H^{2}(M ; \mathbb{Z})$. For any fixed $x \in H_{1}(M ; \mathbb{Z})$, the set $\Gamma_{\tau}^{-1}(x)$ of classes of 2-plane fields $\xi$ mapping to $x$ has a canonical $\mathbb{Z}$-action and is isomorphic to $\mathbb{Z} / d(\xi)$ as a $\mathbb{Z}$-space.

Note that $d(\xi)$ equals twice the divisibility of $x$, so it is independent of the choice of $\xi \in \Gamma_{\tau}^{-1}(x)$.

Proof. Since $\tau$ identifies each tangent space of $M$ with $\mathbb{R}^{3}$ (with the standard orientation and inner product), oriented 2-plane fields $\xi$ on $M$ correspond to their orthogonal unit vector fields, or to maps $\varphi_{\xi}: M \rightarrow S^{2}$. By the Thom-Pontrjagin construction [M2], homotopy classes of such maps correspond bijectively to framed cobordism classes of framed links in $M$, and the correspondence sends $\varphi_{\xi}$ to $\varphi_{\xi}^{-1}(p)$ for any regular value $p \in S^{2}$, framed by pulling back an oriented basis of $T_{p} S^{2}$. Since $M$ is oriented by $\tau$, $\varphi_{\xi}^{-1}(p)$ is an oriented cycle. We define $\Gamma_{\tau}(\xi)$ to be the class $\left[\varphi_{\xi}^{-1}(p)\right] \in H_{1}(M ; \mathbb{Z})$, which is independent of $p$ and depends on $\xi$ and $\tau$ only through their homotopy classes. Clearly, as a 2-plane bundle over $M$, $\xi \cong \varphi_{\xi}^{*}\left(T S^{2}\right)$, so $c_{1}(\xi)$ is Poincaré dual to $2 \Gamma_{\tau}(\xi)$.

Now for fixed $x \in H_{1}(M ; \mathbb{Z})$, the set $\Gamma_{\tau}^{-1}(x)$ is identified with the set of framed cobordism classes of framed links representing $x$. Clearly, $\Gamma_{\tau}^{-1}(x)$ is nonempty and has a canonical $\mathbb{Z}$-action, where $n \in \mathbb{Z}$ acts by adding $n$ right twists to the framing. The $\mathbb{Z}$-action on $\Gamma_{\tau}^{-1}(x)$ is obviously transitive. To verify that the stabilizer of a class is $d(\xi) \mathbb{Z}$, fix a nonempty framed link $L$ representing $x$, and let $L^{\prime}$ denote $L$ with $n$ twists added to its framing. Suppose there is a framed cobordism in $I \times M$ between $L$ and $L^{\prime}$, with $L \subset 1 \times M$ and $L^{\prime} \subset 0 \times M$. By gluing $1 \times M$ to $0 \times M$, we get a closed surface in $S^{1} \times M$ with self-intersection number $n$. Let $\alpha \in H_{2}\left(S^{1} \times M ; \mathbb{Z}\right)$ denote the corresponding homology class with $\alpha^{2}=n$, and let $\lambda=\left[S^{1}\right] \times x \in H_{2}\left(S^{1} \times M ; \mathbb{Z}\right)$. Then $\alpha-\lambda$ intersects $0 \times M$ trivially, so it pulls back to $H_{2}(M)$. Thus, $(\alpha-\lambda)^{2}=\lambda^{2}=0$, and $n=((\alpha-\lambda)+\lambda)^{2}=2(\alpha-\lambda) \cdot \lambda$ in $S^{1} \times M$. But this equals $2(\alpha-\lambda) \cdot x=\left\langle\alpha-\lambda, c_{1}(\xi)\right\rangle$ in $M$. Hence, $n$ is divisible by $d(\xi)$. Conversely, we can find a class $\beta \in H_{2}(M)$ with $\left\langle\beta, c_{1}(\xi)\right\rangle=d(\xi)$, and construct a framed cobordism as above with $\alpha-\lambda=\beta$ and $n=d(\xi)$. We conclude that the stabilizer of the framed cobordism class of $L$ is $d(\xi) \mathbb{Z}$, completing the proof. 
Remark. This proposition can be interpreted in terms of $\operatorname{spin}^{c}$-structures. A 2-plane field $\xi$ as above determines a complex structure on $T M \oplus \mathbb{R}$ (by splitting it into a pair of complex line bundles), and hence, a $\operatorname{spin}^{c}$-structure on $M\left(\right.$ since $\operatorname{Spin}^{c}(3)=U(2)$ acting on $\left.\Lambda^{-}\left(\mathbb{R}^{4}\right) \cong \mathbb{R}^{3}\right)$. A trivialization $\tau$ identifies the set of $\operatorname{spin}^{c}$-structures with $H^{2}(M ; \mathbb{Z})$, and the class assigned to the $\operatorname{spin}^{c}$-structure given by $\xi$ is Poincaré dual to $\Gamma_{\tau}(\xi)$. To see this, note that since $\xi \cong \varphi_{\xi}^{*}\left(T S^{2}\right)$, this procedure for mapping $\xi$ into $H^{2}(M ; \mathbb{Z})$ is the pull-back under $\varphi_{\xi}$ of the corresponding procedure on $T S^{2} \oplus \mathbb{C}$, which has $c_{1}=2\left[S^{2}\right]$ and hence determines the unique $\operatorname{spin}^{c}$-structure on $T \mathbb{R}^{3} \mid S^{2}$ corresponding to $\left[S^{2}\right] \in H^{2}\left(S^{2} ; \mathbb{Z}\right)$. An immediate corollary is that the canonical map from 2-plane fields on $M$ to $\operatorname{spin}^{c}$-structures is surjective, and the preimage of each $\operatorname{spin}^{c}$-structure is a $\mathbb{Z}$-space isomorphic to $\mathbb{Z} / d$, where $d$ is the divisibility of the Chern class of the structure. The grading of the Seiberg-Witten-Floer theory of the $\operatorname{spin}^{c}$-structure is also $\mathbb{Z} / d$. Our invariant $\widetilde{\Theta}$ provides a connection between these $\mathbb{Z}$-spaces, since it is defined via the obstruction $\frac{1}{4}\left(c_{1}^{2}-2 \chi-3 \sigma\right)$ to extending an almost-complex structure over a closed 4-manifold $X$ from $X-p$. The same quantity gives the Seiberg-Witten index of the corresponding $\operatorname{spin}^{c}$-structure on $X$. Recently, Kronheimer and Mrowka $[\mathrm{KM}]$ have defined an explicit bijection between homotopy classes of 2-plane fields and Seiberg-Witten-Floer groups.

We wish to define the invariant picking out the 3 -dimensional obstruction (from $\mathbb{Z} / d(\xi)$ up to translation) by making $(M, \xi)$ bound an almost-complex 4-manifold $X$, defining the obstruction to be $c_{1}^{2}(X)-$ $2 \chi(X)-3 \sigma(X)$ (where $\chi$ and $\sigma$ denote the topological Euler characteristic and signature), and proving invariance by observing that this quantity vanishes for closed, almost-complex 4-manifolds. However, we need additional structure to define the first term, since there is no natural quadratic form on $H^{2}(X) \cong H_{2}(X, \partial X)$ in general.

The proof of Proposition 4.1 actually shows that it makes sense to talk about a framing on a homology class $x \in H_{1}(M ; \mathbb{Z})$ in an oriented 3-manifold $M$ (not necessarily connected), modulo twice the divisibility of $x$, by picking a framed cobordism class mapping to $x$. Equivalently, one frames an oriented link representing $x$, then observes that any other such nonempty representative of $x$ is uniquely framed (modulo twice the divisibility of $x$ and allowing twists to transfer between link components in each component of $M$ ) via a framed cobordism. Now consider a smooth 1-cycle $\gamma$ carried by an oriented link $L=\amalg \gamma_{i}$ in $M$, which we define to be an integer linear combination $\sum k_{i} \gamma_{i}$ of components of $L$. Any framing on $L$ determines a framing on $[\gamma] \in H_{1}(M ; \mathbb{Z})$ (but not conversely, in general) by replacing each $\gamma_{i}$ with $\left|k_{i}\right|$ parallel copies of $\gamma_{i}$, determined by the given framing on $\gamma_{i}$. Now if $z$ is a relative rational 2 -cycle in an oriented 4-manifold pair $\left(X^{4}, \partial X^{4}\right)$, with $\partial z$ a smooth 1-cycle in $\partial X$, we can define the square of $z, Q_{f}(z) \in \mathbb{Q}$, relative to a framing $f$ on the link $L$ carrying $\partial z$, by adding 2-handles to $X$ along $L$ with framings given by $f$. Then $z$ will extend canonically to a rational cycle $\hat{z}$ in $\widehat{X}=X \cup 2$-handles, and we can define $Q_{f}(z)$ to be $\hat{z}^{2}$ in the intersection pairing on $H_{2}(\widehat{X} ; \mathbb{Q})$. This quantity does not change if we break up each $k_{i} \gamma_{i}$ into $\left|k_{i}\right|$ parallel copies of $\gamma_{i}$ using the framing $f$, since the 2-handles attached to the parallel copies will lie inside the 2-handle attached to $\gamma_{i}$. Similarly, $Q_{f}(z)$ is preserved if we change $z$ and $(L, f)$ by attaching a framed cobordism in $I \times \partial X$ to $z$ in $X$. If $z$ is an integer cycle and we change it within its class in $H_{2}(X, \partial X ; \mathbb{Z})$ keeping $\partial z$ fixed, then $[\hat{z}]$ changes by a class in $H_{2}(\partial X ; \mathbb{Z})$, so $Q_{f}(z) \in \mathbb{Z}$ reduces modulo twice the divisibility of $[\partial z]$ to a class $Q_{f}[z]$ depending only on $[z] \in H_{2}(X, \partial X ; \mathbb{Z})$ and $f$ on $[\partial z]$. Similarly, if $z$ is rational and $[\partial z]$ vanishes in $H_{1}(\partial X ; \mathbb{Q})$ then $Q_{f}(z) \in \mathbb{Q}$ is determined by $[z] \in H_{2}(X, \partial X ; \mathbb{Q})$ and $f$ on $[\partial z] \in H_{1}(\partial X ; \mathbb{Z})$.

Now if $x \in H_{1}(M ; \mathbb{Z})$ is rationally trivial, then we can assign numbers to framings $f$ on $x$ just as we do for framings on nullhomologous knots. Simply define $q_{f}(x) \in \mathbb{Q}$ to be $Q_{f}(z)$ for any rational cycle $z$ in $(I \times M,\{1\} \times M)$ with $[\partial z]=x$. As above, this only depends on $x$ and $f$. Clearly, if $\gamma$ is a $\mathbb{Z}$-nullhomologous knot, then $q_{f}[\gamma]$ is the usual integer assigned to the framing $f$ on $\gamma$. In general, $q_{f}(k x)=k^{2} q_{f}(x)$. Adding a right twist to $f$ (on a link component with multiplicity 1 ) adds 1 to $q_{f}(x)$ (and it adds $k_{i}^{2}$ if the multiplicity is $k_{i}$ ), so $q_{f}(x) \bmod 1$ is independent of $f$. In fact, it equals the square of $x$ under the linking pairing, the well-known $\mathbb{Q} / \mathbb{Z}$-valued symmetric bilinear form on the torsion subgroup of $H_{1}(M)$. It follows immediately that $q_{f}(x) \in \frac{1}{k} \mathbb{Z}$, where $k$ is the order of $x$.

For some classes in $H_{2}\left(X^{4}, \partial X^{4}\right)$, there is a canonical square, independent of a choice of framing. By the long exact sequence for $(X, \partial X)$ and the fact that the image of $H_{2}(\partial X)$ in $H_{2}(X)$ is annihilated by the intersection pairing, we see that $\operatorname{ker}\left(\partial_{*}: H_{2}(X, \partial X ; \mathbb{Q}) \rightarrow H_{1}(\partial X ; \mathbb{Q})\right)$ inherits a pairing. Thus, if $z$ 
is a rational 2-cycle in $(X, \partial X)$ whose boundary is a rationally nullhomologous smooth 1-cycle in $\partial X$, then $z^{2} \in \mathbb{Q}$ is canonically defined. To compare $z^{2}$ with $Q_{f}(z)$ for any framing $f$ on a link carrying $\partial z$, we form $\hat{z}$ in $\widehat{X}$ as above. If $z_{1}$ is a rational 2-chain in $\partial X$ with $\partial z_{1}=\partial z$, then $\hat{z}=\left(z-z_{1}\right)+\hat{z}_{1}$. Squaring, we obtain $\hat{z}^{2}=\left(z-z_{1}\right)^{2}+\hat{z}_{1}^{2}$ (the cross-term vanishes since $z-z_{1}$ can be pushed into int $X$ ), or $Q_{f}(z)=z^{2}+q_{f}[\partial z]$. In particular, the right-hand side is integral if $z$ is.

We can now define invariants $\Theta_{f}$ and $\theta$ that partially capture the 3 -dimensional uniqueness obstruction of plane fields. We denote any Poincaré duality isomorphism by $P D$.

Definition 4.2. Let $\xi$ be an oriented 2-plane field on a closed, oriented 3-manifold $M$ (not necessarily connected). We say that $(M, \xi)$ is the almost-complex boundary of a compact, almost-complex 4-manifold $X$ if $\partial X=M$ (as an oriented manifold) and $\xi$ is the field of complex lines in $T M \subset T X \mid M$. If so, then for any framing $f$ on $P D c_{1}(\xi) \in H_{1}(M ; \mathbb{Z})$, let $\Theta_{f}(\xi)=Q_{f}\left(P D c_{1}(X)\right)-2 \chi(X)-3 \sigma(X) \in \mathbb{Z} / 2 d(\xi)$. If $c_{1}(\xi)$ is a torsion class, let $\theta(\xi)=\left(P D c_{1}(X)\right)^{2}-2 \chi(X)-3 \sigma(X) \in \mathbb{Q}$.

Proposition 4.3. When $\theta(\xi)$ is defined, we have $\Theta_{f}(\xi)=\theta(\xi)+q_{f}\left(P D c_{1}(\xi)\right) \in \mathbb{Z}$. In particular, $\theta(\xi)$ is congruent mod 1 to $-q_{f}\left(P D c_{1}(\xi)\right)$, which is an integer divided by the (finite) order of $c_{1}(\xi)$.

For any $(M, \xi)$ as in Definition 4.2 , the required $X$ can be constructed by applying the following lemma to each component of $M$. The invariants $\Theta_{f}(\xi)$ and $\theta(\xi)$ are independent of $X$ by the next theorem. Although we do not need $X$ to be spin or a 2-handlebody, we can arrange these additional conditions with no extra work.

Lemma 4.4. Let $\xi$ be an oriented 2-plane field on a closed, connected, oriented 3-manifold M. Fix a spin structure $s$ on $M$. Then $(M, \xi, s)$ is the almost-complex, spin boundary of a compact, almost-complex, spin 4-manifold $X$ consisting of a 0-handle and 2-handles.

Proof. By [Ka] (for example), we can realize $(M, s)$ as the spin boundary of a spin handlebody $X^{\prime}$ with only 0- and 2-handles. Define a complex structure on $T X^{\prime} \mid M$ by declaring $\xi$ and a complementary trivial bundle to be complex line bundles. Since $S O(4) / U(2) \approx S^{2}$ is simply connected, we can extend the almost-complex structure over the cocores of the 2-handles, and then over the complement of the center $p$ of the 0-handle. Now $c_{1}\left(X^{\prime}\right) \in H^{2}\left(X^{\prime} ; \mathbb{Z}\right) \cong H^{2}\left(X^{\prime}-\{p\} ; \mathbb{Z}\right)$ is defined. According to [HH], the 4 -dimensional obstruction to defining an almost-complex structure on a closed, oriented 4-manifold $W$ is given by $\frac{1}{4}\left(c_{1}^{2}(W)-2 \chi(W)-3 \sigma(W)\right)$, and this can be written as a sum of local obstructions at isolated singularities. But $S^{2} \times S^{2}$ admits a complex structure with $c_{1}^{2}=8$, and an almost complex structure in the complement of a point with $c_{1}=0$. Thus, we can add \pm 1 to the index of a singularity by forming the connected sum with $S^{2} \times S^{2}$ there. Now for a suitable connected sum $X=X^{\prime} \# n S^{2} \times S^{2}$, the obstruction at $p$ will vanish, yielding our required almost-complex spin manifold.

Theorem 4.5. Let $\xi$ be an oriented 2-plane field on a closed, oriented 3-manifold $M$ (not necessarily connected), and let $f$ be a framing on $P D c_{1}(\xi)$. Then $\Theta_{f}(\xi) \in \mathbb{Z} / 2 d(\xi)$ depends only on $M, f$ and the homotopy class $[\xi]$ of $\xi$. If $c_{1}(\xi)$ is a torsion class, then $\theta(\xi) \in \mathbb{Q}$ depends only on $M$ and $[\xi]$. Both invariants are independent of the orientation of $\xi$, reverse sign if the orientation of $M$ is reversed, and add under disjoint union. Adding a right twist to $f$ increases $\Theta_{f}(\xi)$ by 1. If $\varphi: M \rightarrow M^{\prime}$ is an orientation-preserving diffeomorphism, then $\Theta_{\varphi_{*} f}\left(\varphi_{*} \xi\right)=\Theta_{f}(\xi)$ and $\theta\left(\varphi_{*} \xi\right)=\theta(\xi)$ (when defined).

Proof. Given $\left(M, \xi_{0}\right)$ as in the theorem and $\xi_{1}$ homotopic to $\xi_{0}$, let $X_{0}$ and $X_{1}$ be any compact, almostcomplex manifolds with almost-complex boundaries $\left(M, \xi_{0}\right)$ and $\left(\bar{M}, \xi_{1}\right)$, respectively, where $\bar{M}$ denotes $M$ with reversed orientation. Glue $X_{0}$ and $X_{1}$ to opposite boundary components of $I \times M$ to obtain a closed, oriented manifold $W$, and extend the almost-complex structures across $I \times M$ to make $W$ almostcomplex, using a homotopy from $\xi_{0}$ to $\xi_{1}$. Clearly, $\chi(W)=\chi\left(X_{0}\right)+\chi\left(X_{1}\right)$ and similarly for $\sigma$. Also, $c_{1}^{2}(W)-2 \chi(W)-3 \sigma(W)=0$. To analyze $\theta$, we assume that $c_{1}\left(\xi_{0}\right)$ is a torsion class and let $\theta_{i}=\theta\left(\xi_{i}\right)$ be as in Definition 4.2, defined using the manifold $X_{i}$. Now rationally, $P D c_{1}(W)$ can be written as $\alpha_{0}+\alpha_{1}$, where $\alpha_{i} \in H_{2}(W ; \mathbb{Q})$ pulls back to $H_{2}\left(X_{i} ; \mathbb{Q}\right)$ and then maps to $P D c_{1}\left(X_{i}\right)$ in $H_{2}\left(X_{i}, \partial X_{i} ; \mathbb{Q}\right)$. It follows that $c_{1}^{2}(W)=\left(P D c_{1}\left(X_{0}\right)\right)^{2}+\left(P D c_{1}\left(X_{1}\right)\right)^{2}$, so the above formula on $W$ implies that $\theta_{0}+\theta_{1}=0$. Since $X_{0}$ and $X_{1}$ were chosen independently, $\theta_{0}$ depends only on $M$ and the homotopy class of $\xi_{0}$, and $\theta_{1}=-\theta_{0}$ is the result of reversing the orientation of $M$. Reversing the orientation of $\xi_{i}$ corresponds to 
conjugating the almost-complex structure on $X_{i}$, which fixes $\left(P D c_{1}\left(X_{i}\right)\right)^{2}$. A similar argument applies to $\Theta_{f}$ in the general case, once we observe that for any framing $f$ on $P D\left(c_{1}(\xi)\right), c_{1}^{2}(W)$ reduces $\bmod 2 d(\xi)$ to $Q_{f}\left(P D c_{1}\left(X_{0}\right)\right)+Q_{f}\left(P D c_{1}\left(X_{1}\right)\right)$. The last sentence of the theorem follows immediately from the observation that if $(M, \xi)$ is the almost-complex boundary of $X$, then $\left(M^{\prime}, \varphi_{*} \xi\right)$ is the almost-complex boundary of $X$ by the gluing $\operatorname{map} \varphi$.

Corollary 4.6. For $i=1,2$, let $\left(M_{i}, \xi_{i}\right)$ be the holomorphically fillable contact 3-manifold obtained by contact surgery on a Legendrian knot $K_{i}$ in $S^{3}$. Suppose there is an orientation-preserving diffeomorphism $\varphi: M_{1} \rightarrow M_{2}$ such that $\varphi_{*}\left(\xi_{1}\right)$ is homotopic to $\xi_{2}$ (with either orientation). Then $t b\left(K_{1}\right)=t b\left(K_{2}\right)$ and $\left|r\left(K_{1}\right)\right|=\left|r\left(K_{2}\right)\right|$.

For example, if $K$ is any Legendrian knot in $S^{3}$ with $t b(K) \geq 2$ (respectively, 4 ), then by adding zig-zags to $K$ we can obtain smoothly equivalent Legendrian knots $K_{i}$ with $t b\left(K_{i}\right)=0$ (respectively 2 ) but distinct values of $\left|r\left(K_{i}\right)\right|$. Contact surgery on these knots will yield noncontactomorphic holomorphically fillable contact structures on the same homology sphere. (In fact, they bound diffeomorphic Stein manifolds with different Chern classes.) Their homotopy classes will be distinguished by the 3-dimensional obstruction $\theta$, but not by the 2-dimensional one $\Gamma_{\tau} \in H_{1}(M ; \mathbb{Z})=0$ - in particular, not by the Chern class, providing new counterexamples to Conjecture 10.3 of [E3]. Similarly, one can realize such examples for any finite cyclic $H_{1}$ by arranging $|r|$ to take more values than the order of $H_{1}$.

Proof. Let $r_{i}$ denote $r\left(K_{i}\right)$ and let $n_{i}=t b\left(K_{i}\right)-1$ denote the surgery coefficient. Then $\left|n_{1}\right|=\left|n_{2}\right|$ is the order of the cyclic group $H_{1}\left(M_{i} ; \mathbb{Z}\right)$. If $n_{i}=0$, then this group is infinite, $\left|r_{i}\right|=d\left(\xi_{i}\right)$, and we are done. Otherwise, the group is finite, $\theta\left(\xi_{i}\right) \in \mathbb{Q}$ is defined, and $\theta\left(\xi_{1}\right)=\theta\left(\xi_{2}\right)$. We compute $\theta\left(\xi_{i}\right)$ using the Stein surface $X_{i}$ obtained by adding a 2-handle to $B^{4}$ along $K_{i}$. Let $\alpha_{i}$ be a generator of $H_{2}\left(X_{i} ; \mathbb{Z}\right) \cong \mathbb{Z}$. Then $\alpha_{i}^{2}=n_{i}$, so the cocore disk $D_{i}$ of the 2 -handle represents $\frac{1}{n_{i}} \alpha_{i} \in H_{2}\left(X_{i}, \partial X_{i} ; \mathbb{Q}\right) \cong \mathbb{Q}$, and $P D c_{1}\left(X_{i}\right)=\frac{r_{i}}{n_{i}} \alpha_{i}$ over $\mathbb{Q}$. Thus, $\theta\left(\xi_{i}\right)=\frac{r_{i}^{2}}{n_{i}}-4-3 \operatorname{sign} n_{i}$. Now it clearly suffices to show that $n_{1}=n_{2}$. If not, then $n_{1}=-n_{2}$, and setting $\theta\left(\xi_{1}\right)=\theta\left(\xi_{2}\right)$ shows that $r_{1}^{2}+r_{2}^{2}=6\left|n_{1}\right|$. Let $x_{i} \in H_{1}\left(M_{i}\right.$; $\left.\mathbb{Z}\right)$ be the generator $\left[\partial D_{i}\right]$. Then $q_{f}\left(x_{i}\right) \equiv-\frac{1}{n_{i}}(\bmod 1)$. (For example, let $z=D_{i}-\frac{1}{n_{i}} \alpha_{i}$, which can be pushed into $I \times M_{i}$.) But for some $k \in \mathbb{Z}$ we have $\varphi_{*}\left(x_{1}\right)=k x_{2}$, so $q_{f_{1}}\left(x_{1}\right)=q_{\varphi_{*} f_{1}}\left(\varphi_{*}\left(x_{1}\right)\right) \equiv k^{2} q_{f_{2}}\left(x_{2}\right)(\bmod 1)$, and therefore $k^{2} \equiv-1 \bmod \left|n_{1}\right|$. Reducing the equation $r_{1}^{2}+r_{2}^{2}=6\left|n_{1}\right| \operatorname{modulo} 3$, we see that $r_{1}$ and $r_{2}$ must be divisible by 3 , and so $n_{1}$ is also divisible by 3 . But the equation $k^{2} \equiv-1(\bmod 3)$ has no solutions, so we have the required contradiction.

Like $\theta$ in the case $d(\xi)=0$, the invariant $\Theta_{f}$ can easily be computed for the boundary of any Stein surface $X$ in standard form. In fact, $P D c_{1}(X)$ is represented by $\sum r\left(K_{i}\right) D_{i}$, where $D_{i}$ is the cocore of the handle of $X$ attached to $K_{i}$. Thus, $Q_{f}\left(P D c_{1}(X)\right)=0$ when $f$ is the 0 -framing on the union of meridians carrying $\sum r\left(K_{i}\right) \partial D_{i}$. To compare $\Theta$ for two different Stein surfaces with a diffeomorphism preserving $c_{1}(\xi)$ between the boundaries, it now suffices to compare the respective 0-framings by constructing a framed cobordism between the corresponding 1-cycles.

We will see that the canonical generator of the $\mathbb{Z}$-action of Proposition 4.1 subtracts 4 from each of $\Theta_{f}$ and $\theta$. Thus, when $c_{1}(\xi)$ is a torsion class, either invariant captures the 3 -dimensional obstruction. When $c_{1}(\xi)$ has infinite order, however, $\theta(\xi)$ is undefined and $\Theta_{f}(\xi) \in \mathbb{Z} / 2 d(\xi)$ only captures the obstruction up to a $2: 1$ ambiguity. We will lift $\Theta_{f}(\xi)$ to an invariant $\tilde{\Theta}(\xi, s, f) \in \mathbb{Z} / 4 d(\xi)$ depending on a choice of spin structure $s$, and this will capture the 3-dimensional obstruction. This lifting is intimately related to the 2-dimensional obstruction $\Gamma_{\tau}(\xi) \in H_{1}(M ; \mathbb{Z}) \cong H^{2}(M ; \mathbb{Z})$. To clarify the relationship between $\Gamma$ and $\tilde{\Theta}$, we now give an alternate definition of $\Gamma$. The new definition is readily computable (e.g., Theorem 4.12), and it clarifies the dependence of $\Gamma$ on the trivialization $\tau$. Note that $\Gamma_{\tau}(\xi)$ should only depend on the restriction of $\tau$ to the 2-skeleton of $M$, or equivalently, on a spin structure. Here and elsewhere, it is convenient to use Milnor's definition [M1] (see also [GS]) of a spin structure on an oriented manifold $M$ as a homotopy class of positively oriented trivializations of $T M$ over the 2 -skeleton of some cell decomposition of $M$, where if $\operatorname{dim} M \leq 2$ we first stabilize $T M$ by summing with a trivial bundle. Note that there is a canonical way to reverse the orientation of a trivialization, allowing us to identify spin structures on $\bar{M}$ with those on $M$. 
Definition 4.7. Let $\xi$ be an oriented 2-plane field on a closed, oriented 3-manifold $M$ (not necessarily connected). Let $v$ be a vector field in $\xi$ whose zero locus, counted with multiplicities, has the form $2 \gamma$ for some smooth 1-cycle $\gamma$ carried by a link $L$ in $M$. The vector field $v$ in $\xi$ determines a spin structure $s$ on $M-L$, and this extends uniquely over $M$ since $v$ vanishes with even multiplicity on $L$. Define $\Gamma(\xi, s)$ to be the class $[\gamma] \in H_{1}(M ; \mathbb{Z})$.

The next proposition shows that $\Gamma(\xi, s)$ is well-defined and depends in a simple way on $s$. To understand this dependence, recall that the group $H^{1}\left(M ; \mathbb{Z}_{2}\right) \cong H_{2}\left(M ; \mathbb{Z}_{2}\right)$ acts freely and transitively on the set $\mathcal{S}(M)$ of spin structures of $M$. The same group acts on $H_{1}(M ; \mathbb{Z})$ via the Bockstein homomorphism $\beta$ in homology induced by the coefficient sequence $\mathbb{Z} \rightarrow \mathbb{Z} \rightarrow \mathbb{Z}_{2}$.

Proposition 4.8. Let $\xi$ be an oriented 2-plane field on a closed, oriented 3-manifold $M$. Then the map $\Gamma(\xi, \cdot): \mathcal{S}(M) \rightarrow H_{1}(M ; \mathbb{Z})$ is well-defined (depending only on $M$ and $\left.[\xi]\right)$ and $H^{1}\left(M ; \mathbb{Z}_{2}\right)$-equivariant. For $M, \tau$ and $\Gamma_{\tau}$ as in Proposition 4.1, $\Gamma_{\tau}(\xi)$ equals $\Gamma(\xi, s)$ where $s$ is the spin structure induced by $\tau$.

Proof. For $i=0,1$, let $v_{i}, L_{i}, \gamma_{i}$ and $s_{i}$ be as in Definition 4.7. Let $\Delta\left(v_{0}, v_{1}\right) \in H^{1}\left(M-\left(L_{0} \cup L_{1}\right) ; \mathbb{Z}\right)$ be the difference class of the nonzero vector fields $v_{i}$ in $\xi \mid M-\left(L_{0} \cup L_{1}\right)$. Then the mod 2 reduction of $\Delta\left(v_{0}, v_{1}\right)$ extends uniquely over $H^{1}\left(M ; \mathbb{Z}_{2}\right)$ as the difference class $\Delta\left(s_{0}, s_{1}\right)$ of the spin structures. Now $\beta P D \Delta\left(s_{0}, s_{1}\right)=\frac{1}{2} \partial P D \Delta\left(v_{0}, v_{1}\right)=\left[\gamma_{0}\right]-\left[\gamma_{1}\right]$, so $\Gamma(\xi, \cdot)$ is well-defined on spin structures induced by vector fields as in Definition 4.7 , and it has the required equivariance on them.

Now any spin structure $s \in \mathcal{S}(M)$ is induced by a trivialization $\tau$ of $T M$, since $\pi_{2}(S O(3))=0$. Using $\tau$, define $\varphi_{\xi}: M \rightarrow S^{2}$ with $\xi \cong \varphi_{\xi}^{*}\left(T S^{2}\right)$ as in the proof of Proposition 4.1. Let $w$ be a vector field on $S^{2}$ with a unique zero, occurring at a regular value $p$ of $\varphi_{\xi}$. Then $v=\varphi_{\xi}^{*}(w)$ is a vector field in $\xi$ vanishing with multiplicity 2 on $\gamma=\varphi_{\xi}^{-1}(p)$. Applying Definition 4.7, we see that $\Gamma\left(\xi, s^{\prime}\right)=\left[\varphi_{\xi}^{-1}(p)\right]=\Gamma_{\tau}(\xi)$, where $s^{\prime}$ is the spin structure on $M$ induced by $v$. But $s^{\prime}$ is the pull-back under $\varphi_{\xi}$ of the unique spin structure on $S^{2}$, which comes from the canonical trivialization of $\mathbb{R}^{3}$. Since this trivialization pulls back to $\tau$, we have $s^{\prime}=s$. In particular, any $s$ is induced by a suitable vector field $v$, so $\Gamma(\xi, s)$ is defined, and $\Gamma(\xi, s)=\Gamma_{\tau}(\xi)$.

Corollary 4.9. Under the above hypotheses, the map $\Gamma(\xi, \cdot): \mathcal{S}(M) \rightarrow H_{1}(M ; \mathbb{Z})$ is determined by its value on any one $s \in \mathcal{S}$, hence, by any vector field $v$ as in Definition 4.7. Its image is $\left\{x \in H_{1}(M ; \mathbb{Z}) \mid\right.$ $\left.2 x=P D c_{1}(\xi)\right\}$, and $\Gamma\left(\xi, s_{0}\right)=\Gamma\left(\xi, s_{1}\right)$ if and only if $\Delta\left(s_{0}, s_{1}\right)$ lifts to $H^{1}(M ; \mathbb{Z})$. Reversing the orientation of either $\xi$ or $M$ reverses the sign of $\Gamma(\xi, s)$ for fixed $s$. $\Gamma$ is preserved by surgeries on $S^{0}$ (addition of 4-dimensional 1-handles), and adds in the obvious way under disjoint union (hence, connected sum). If $\varphi: M \rightarrow M^{\prime}$ is an orientation-preserving diffeomorphism, then $\Gamma\left(\varphi_{*} \xi, \varphi_{*} s\right)=\varphi_{*} \Gamma(\xi, s)$. Under finite coverings, $P D \Gamma$ lifts in the obvious way. For $M$ connected, with fixed $s$ and $q \in M, \Gamma(\cdot, s)$ classifies 2-plane fields on $M-\{q\}$ up to homotopy.

Proof. This is immediate from Definition 4.7 and Proposition 4.8. Note that by the long exact coefficient sequence, $\operatorname{Im} \beta$ is the subgroup of elements of $H_{1}(M ; \mathbb{Z})$ with order at most 2 , and $\operatorname{ker}(\beta \circ P D)$ consists of those classes in $H^{1}\left(M ; \mathbb{Z}_{2}\right)$ with integer lifts. The behavior of $\Gamma$ under orientation reversals is the same as that of $P D c_{1}(\xi)$. (Reversing $[M]$ reverses the sign of $P D$.) For the last sentence, extend $s$ to a trivialization $\tau$ and note that any 2-plane field on $M-\{q\}$ extends over $M$, since the corresponding map $S^{2} \rightarrow S^{2}$ is nullhomologous. Clearly, $P D \Gamma(\cdot, s)=P D \Gamma_{\tau}(\cdot)$ is the 2-dimensional uniqueness obstruction for oriented 2-plane fields on $M$, so it classifies 2-plane fields on $M-\{q\}$.

Remarks.

1) Kuperberg ([Ku] Section 2.1) constructs an invariant $c$ equivalent to $\Gamma(\xi, s)$ directly from homotopy theory, and essentially also obtains the above invariance, kernel and image of $\Gamma(\xi, \cdot)$.

2) A spin structure $s$ on $M$ determines a $\operatorname{spin}^{c}$-structure by the inclusion $\operatorname{Spin}(3) \subset \operatorname{Spin}^{c}(3)$. From our viewpoint, this $\operatorname{spin}^{c}$-structure is the one canonically associated to a trivialization $\tau$ extending $s$. By the remark following Proposition 4.1, it follows that $\Gamma(\xi, s)$ is the difference class of the $\operatorname{spin}^{c}$-structures associated to $\xi$ and $s$.

3) Here is yet another interpretation of $\Gamma(\xi, s)$, suggested by D. Freed. The plane field $\xi$ determines an $S O(2)$-subbundle of the tangent $S O(3)$-principal bundle of $M$. A spin structure $s$ lifts the latter to 
an $S U(2)$-bundle, in which $\xi$ determines a $U(1)$-subbundle $\tilde{\xi}$. Then $\Gamma(\xi, s)=P D c_{1}(\tilde{\xi})$. To verify this, fix a trivialization $\tau$ extending $s$, write $\xi \cong \varphi_{\xi}^{*}\left(T S^{2}\right)$ as above, then note that $c_{1}(\tilde{\xi})=\varphi_{\xi}^{*} c_{1}\left(\widetilde{T S^{2}}\right)=\varphi_{\xi}^{*}\left[S^{2}\right]$ is dual to $\left[\varphi_{\xi}^{-1}(p)\right]=\Gamma(\xi, s)$, as required.

Corollary 4.10. Let $\xi$ be an oriented 2-plane field on a closed, oriented 3-manifold $M$. Then $\xi$ is homotopic to itself with reversed orientation if and only if $c_{1}(\xi)=0$.

This rules out the case where $c_{1}(\xi)$ has order 2 .

Proof. Given such a homotopy, then for a fixed spin structure $s$ on $M$ we have $\Gamma(\xi, s)=-\Gamma(\xi, s)$, so $P D c_{1}(\xi)=2 \Gamma(\xi, s)=0$. The converse is obvious by direct construction, trivializing $\xi$.

To compute $\Gamma(\xi, s)$ for the almost-complex boundary $(M, \xi)$ of a compact Stein surface $X$, it is convenient to express spin structures in terms of characteristic sublinks. (See, for example, [GS].) We can assume that $X$ is presented in standard form (Definition 2.1). Then there is a canonical way to surger out the 1-handles of $X$ to obtain a 4 -manifold $X^{*}$ with $\partial X^{*}=M$, such that $X^{*}$ is obtained from $B^{4}$ by adding 2-handles along a framed link $L$ in $S^{3}$. This link is obtained by stretching the box in the plane, forming an annulus by gluing together its lateral edges in the obvious way (Figure 26). Each 2-handle of $X$ becomes a 2-handle of $X^{*}$ with the same framing, and the remaining 2-handles of $X^{*}$ form a sublink $L_{0} \subset L$ consisting of a 0 -framed unknot for each 1-handle $h$. (Curves that formerly ran over $h$ will now link the unknot.) Clearly, $H_{2}\left(X^{*} ; \mathbb{Z}_{2}\right)$ corresponds bijectively to the set of all sublinks $L^{\prime}$ of $L$, with each $L^{\prime}$ mapping to the homology class determined by the cores of the corresponding 2-handles. Fix an orientation on $L$. For components $K, K^{\prime}$ of $L$, we define the linking number $\ell k\left(K, K^{\prime}\right)$ to be the usual linking number if $K \neq K^{\prime}$ and to be the framing of $K$ if $K=K^{\prime}$. We use bilinearity to extend the definition to $\ell k\left(\gamma, \gamma^{\prime}\right)$ for $\gamma, \gamma^{\prime}$ smooth 1-cycles carried by $L$, and use the same formalism mod 2 for sublinks of $L$. These pairings correspond to the intersection pairings of $X^{*}$ over $\mathbb{Z}$ and $\mathbb{Z}_{2}$, respectively.
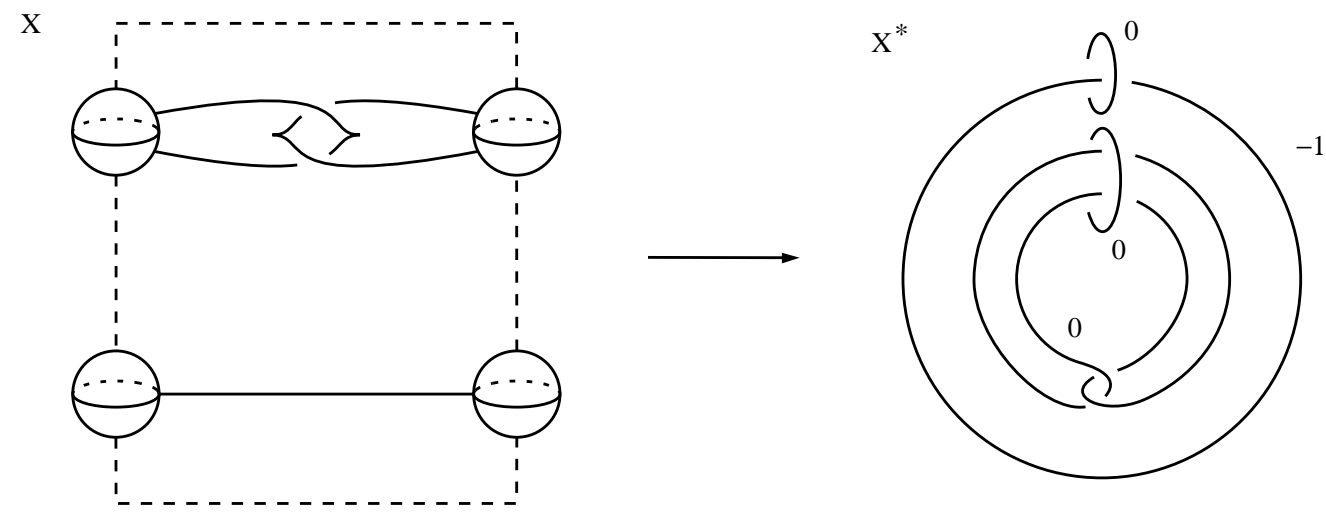

FiguRE 26

Definition 4.11 [Ka]. A sublink $L^{\prime}$ of $L$ is characteristic if for each component $K$ of $L$, the framing of $K$ is congruent modulo 2 to $\ell k\left(K, L^{\prime}\right)$.

By the $\mathrm{Wu}$ formula, a sublink $L^{\prime}$ is characteristic if and only if it corresponds to a class in $H_{2}\left(X^{*} ; \mathbb{Z}_{2}\right)$ mapping to $P D w_{2}\left(X^{*}\right) \in H_{2}\left(X^{*}, M ; \mathbb{Z}_{2}\right)$. It is not hard to show (for example, [GS]) that $\mathcal{S}(M)$ maps bijectively to the set of characteristic sublinks of $L$ by sending a spin structure $s$ to the link $L^{\prime}$ corresponding to $P D w_{2}\left(X^{*}, s\right) \in H_{2}\left(X^{*} ; \mathbb{Z}_{2}\right)$, the dual of the Stiefel-Whitney class of $X^{*}$ relative to $s$. The spin structure $s$ corresponding to $L^{\prime}$ is then characterized by the fact that if we attach a 2-handle to $X^{*}$ along any knot $K$ in $S^{3}-L$ with framing $n$, the structure $s$ will extend over the new handle if and only if $n \equiv \ell k\left(K, L^{\prime}\right) \bmod 2$. Note that the spin structure near $K$ that extends over the 2-handle is the one not induced by the normal framing and tangent vectors to $K$, since the tangent vector field to $\partial D^{2} \subset \mathbb{R}^{2}$ has odd degree. The difference class $\Delta\left(s_{0}, s_{1}\right) \in H^{1}\left(M ; \mathbb{Z}_{2}\right) \cong H_{2}\left(\partial X^{*} ; \mathbb{Z}_{2}\right) \subset H_{2}\left(X^{*} ; \mathbb{Z}_{2}\right)$ corresponds to the difference of the characteristic sublinks for $s_{0}$ and $s_{1}$. There is a simple procedure (see [GS], for example) for following spin structures through sequences of Kirby moves via their characteristic sublinks. 
Theorem 4.12. Let $X$ be a compact Stein surface in standard form, with $\partial X=(M, \xi)$, and $X^{*}, L=$ $K_{1} \cup \cdots \cup K_{n}$ and $L_{0}$ as above. Let $\left\{\alpha_{1}, \ldots, \alpha_{n}\right\} \subset H_{2}\left(X^{*} ; \mathbb{Z}\right)$ be the basis determined by $\left\{K_{1}, \ldots, K_{n}\right\}$. Let $s$ be a spin structure on $M$, represented by a characteristic sublink $L^{\prime}$ of $L$. Then $P D \Gamma(\xi, s)$ is the restriction to $M$ of the class $\rho \in H^{2}\left(X^{*} ; \mathbb{Z}\right)$ whose value on each $\alpha_{i}$ is the integer

$$
\left\langle\rho, \alpha_{i}\right\rangle=\frac{1}{2}\left(r\left(K_{i}\right)+\ell k\left(K_{i}, L_{0}+L^{\prime}\right)\right) .
$$

Here we define $r\left(K_{i}\right)$ to be 0 if $K_{i}$ is in $L_{0}$, and otherwise to be the rotation number of the corresponding Legendrian knot in the diagram for $X$. The theorem still holds if we replace $L_{0}+L^{\prime}$ in the formula by any smooth 1-cycle (independent of $i$ ) carried by $L$ and agreeing with $L_{0}+L^{\prime} \bmod 2$. This is because adding $2 \gamma$ to $L_{0}+L^{\prime}$ changes $\left\langle\rho, \alpha_{i}\right\rangle$ by $\ell k\left(K_{i}, \gamma\right)=\alpha_{i} \cdot \alpha_{\gamma}$, where $\alpha_{\gamma} \in H_{2}\left(X^{*} ; \mathbb{Z}\right)$ is the class determined by $\gamma$, so $\rho$ changes by $P D \alpha_{\gamma}$ in $H^{2}\left(X^{*}, M ; \mathbb{Z}\right) \cong H_{2}\left(X^{*} ; \mathbb{Z}\right)$, leaving $\rho \mid M$ unchanged. Similarly, $2 \rho$ restricts to $c_{1}(\xi)$ as required. (Furthermore, the equivariance of Proposition 4.8 can be verified directly.)

Corollary 4.13. Let $K$ be a Legendrian knot in $\# S^{1} \times S^{2}$, presented in standard form as in Definition 2.1. Then $t b(K)+r(K)+1$ is congruent modulo 2 to the number of times $K$ crosses 1-handles.

Proof. Add a 2-handle along $K$ to obtain a Stein surface $X$. By Theorem 4.12, $r(K)+\ell k\left(K, L_{0}+L^{\prime}\right)$ must be even for any characteristic sublink $L^{\prime}$ of $L$ as above. By Definition $4.11, \ell k\left(K, L^{\prime}\right)$ is congruent mod 2 to the framing of $K, t b(K)-1$. Since $\ell k\left(K, L_{0}\right)$ is congruent to the number of times $K$ crosses 1-handles, the corollary follows immediately.

Example 4.14. Consider the Stein surface $X_{p}(p \geq 1)$ shown in Figure 27(a). This is obtained by adding a 2 -handle to $S^{1} \times D^{3}$ along a Legendrian knot $K$ that runs $2 p$ times over the 1-handle. Thus, $w(K)=2 p-1$. There are $2 p-2$ left cusps, half oriented upward and the other half downward, so $\lambda_{+}=\lambda_{-}=\rho_{+}=\rho_{-}=p-1$. Thus, the framing of the 2-handle is $t b(K)-1=0$, and $r(K)=0$. The 2-handlebody $X_{p}^{*}$ (Figure 27(b)) admits a unique spin structure, whose restriction $s$ to $M_{p}=\partial X_{p}^{*}=\partial X_{p}$ is given by the empty characteristic sublink. Using Theorem 4.12 , it is easy to calculate that $\Gamma(\xi, s)=p \mu$, where $\mu$ is the meridian of $K$ in $H_{1}\left(M_{p} ; \mathbb{Z}\right) \cong \mathbb{Z} / 2 p \oplus \mathbb{Z} / 2 p$ (which is generated by the two meridians in $\partial X_{p}^{*}$ ). Now observe that $X_{p}^{*}$ admits an involution $\varphi$ that interchanges the two 2-handles but preserves the orientation and $s$. Since $\varphi_{*} \Gamma(\xi, s) \neq \Gamma(\xi, s)$, we conclude that the holomorphically fillable positive contact structures $\xi$ and $\varphi_{*} \xi$ on $M_{p}$ are not homotopic as 2-plane fields. In particular, they are not isotopic (although they are contactomorphic via $\varphi$ ). However, they are not distinguished by the other homotopy invariants, since $c_{1}(\xi)=c_{1}\left(\varphi_{*} \xi\right)=0$ and $\theta(\xi)=\theta\left(\varphi_{*} \xi\right)=-2$. Figure 27(c) shows that $M_{p}$ is Seifert fibered over $S^{2}$ with 3 multiple fibers (cf. Section 5). In particular, it has no incompressible tori, hence, no Giroux torsion. The manifold $X_{1}$ is the disk bundle over $\mathbb{R} P^{2}$ with (twisted) Euler number -2 , so $M_{1}$ is diffeomorphic to the projectivization of $T^{*} \mathbb{R} P^{2}$. Equivalently, $M_{1}$ is $S^{3}$ in the quaternions modulo left multiplication by the order 8 subgroup generated by $\{i, j\}$.

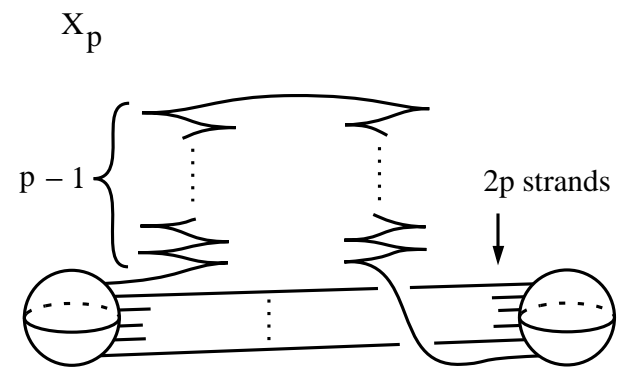

(a)

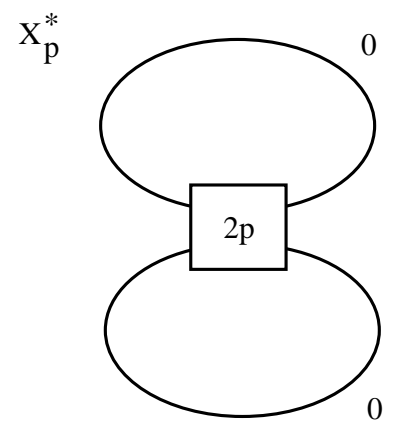

(b)

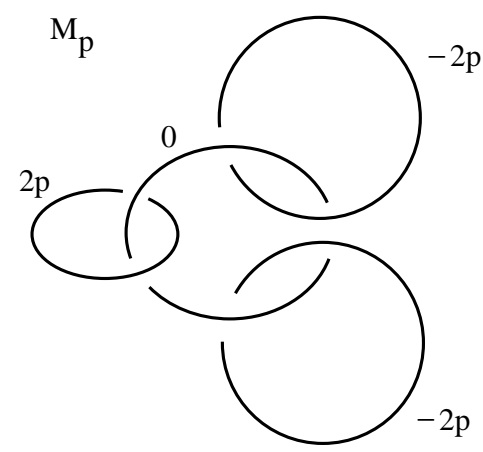

(c)

FigURE 27 
Proof of Theorem 4.12. For convenience, we identify $M=\partial X$ with $\partial X^{*}$, and use the same symbol to denote $K_{i} \subset L-L_{0}$ and the corresponding knot in the diagram for $X$. We let $\nu L$ denote the tubular neighborhood of $L$ in which the 2-handles of $X^{*}$ intersect $S^{3}$. As in the proof of Proposition 2.3, we use the vector field $\frac{\partial}{\partial x}$ to define a complex trivialization of $T X$ over the 0 - and 1-handles. The obstruction to extending this over $X$ is the relative Chern class, dual to $\sum r\left(K_{i}\right) D_{i}$, where $i$ ranges over the 2-handles of $X$ and $D_{i} \subset \nu L$ is a cocore disk of the handle of $X$ attached along $K_{i}$. The trivialization determines a spin structure on $X-\coprod D_{i}$ whose restriction to $M-\coprod \partial D_{i}$ we denote by $s_{0}$. Since $s_{0}$ is determined by $\frac{\partial}{\partial x}$ and $\frac{\partial}{\partial z}$ in the box containing the diagram for $X$, it is characterized in $\partial X^{*}$ by extending over a 2-handle attached to a knot $K \subset S^{3}-\nu L$ if and only if the framing is congruent $\bmod 2$ to $\ell k\left(K, L_{0}\right)$. (Note that a 0 -framed meridian $\mu \subset \partial X^{*}$ of a component of $L_{0}$ corresponds to a horizontal curve crossing the corresponding 1-handle in the original diagram, with framing induced by $\frac{\partial}{\partial x}$. The structure $s_{0}$ will not extend over a 2-handle attached to $\mu$ with this framing, due to the odd degree of a tangent vector field to $\partial D^{2}$.) Now add a collar $I \times M$ to $X$, gluing $0 \times M$ to $\partial X$. If we put the above spin structure on $X-\amalg D_{i}$ and the given structure $s$ on $1 \times M$, then the obstruction to extending to a spin structure on $I \times M \cup_{\partial X} X$ will be dual to a $\mathbb{Z}_{2}$-cycle that lifts to an integer chain of the form $c=\sum r\left(K_{i}\right) D_{i}+z_{1}$, where $z_{1}$ is a chain in $I \times M$. If we identify $z_{1}$ with a chain in $M=\partial X^{*}$, we can assume that its intersection with the 2-handles is a subchain $z_{2}$ that is a linear combination of core disks. Since the spin structure on $0 \times\left(M-\coprod \partial D_{i}\right)$ is given by $s_{0}$, the chain $z_{1}$ represents $P D \Delta\left(s, s_{0}\right) \in H_{2}\left(M, \amalg \partial D_{i} ; \mathbb{Z}_{2}\right)$. After inclusion in $H_{2}\left(X^{*}, B^{4} ; \mathbb{Z}_{2}\right) \cong H_{2}\left(X^{*} ; \mathbb{Z}_{2}\right)$, this class corresponds to the sublink $L_{0}+L^{\prime}$. To verify this, recall that $s_{0}$ and $s$ extend over a 2-handle attached to a knot $K$ in $S^{3}-\nu L$ if and only if the framing is congruent $\bmod 2$ to $\ell k\left(K, L_{0}\right)$ or $\ell k\left(K, L^{\prime}\right)$, respectively. Thus, $s_{0}$ and $s$ agree on $K$ if and only if $\ell k\left(K, L_{0}+L^{\prime}\right)$ vanishes mod 2. By definition, the obstruction $\Delta\left(s, s_{0}\right)$ then satisfies $\left\langle\Delta\left(s, s_{0}\right), K\right\rangle=\ell k\left(L_{0}+L^{\prime}, K\right)$ over $\mathbb{Z}_{2}$ for all $K$. We conclude that in $H_{2}\left(X^{*} ; \mathbb{Z}_{2}\right)$, the class corresponding to $L_{0}+L^{\prime}$ agrees with $P D \Delta\left(s, s_{0}\right)$ when paired with any element of $H_{2}\left(B^{4}, S^{3}-\nu L ; \mathbb{Z}_{2}\right) \cong H_{2}\left(X^{*}, M ; \mathbb{Z}_{2}\right)$, so the classes are equal.

To relate this construction to Definition 4.7 of $\Gamma$, we observe that $s$ is determined by a vector field $v$ in $\xi$ that we construct from $\frac{\partial}{\partial x}$ by twisting along $z_{1}$ - that is, we define $v$ by the formula $P D \Delta\left(v, \frac{\partial}{\partial x}\right)=\left[z_{1}\right] \in$ $H_{2}\left(M, \partial z_{1} ; \mathbb{Z}\right)$. Since $c$ reduces mod 2 to a cycle in $I \times M \cup_{\partial X} X$, we have $\partial c=2 \gamma$ for some smooth 1-cycle $\gamma$ in $S^{3}-L$. Thus, $\partial z_{1}=2 \gamma-\sum r\left(K_{i}\right) \partial D_{i}$. Since $\frac{\partial}{\partial x}$ extends to a vector field in $\xi$ on $M$ with zero locus $\sum r\left(K_{i}\right) \partial D_{i}, v$ is defined on $M$ with zero locus $2 \gamma$. By Definition 4.7, $\Gamma(\xi, s)=[\gamma] \in H_{1}(M ; \mathbb{Z})$. But $z_{1}-z_{2}$ is a chain in $S^{3}-L$ with $\partial\left(z_{1}-z_{2}\right)=2 \gamma-\sum r\left(K_{i}\right) \partial D_{i}-\partial z_{2}$, so the right-hand side is nullhomologous in $S^{3}-L$, and in the free abelian group $H_{1}\left(S^{3}-L ; \mathbb{Z}\right),[\gamma]$ is given by the uniquely defined class $\frac{1}{2}\left(\sum r\left(K_{i}\right)\left[\partial D_{i}\right]+\left[\partial z_{2}\right]\right)$. Now we apply the map $h: H_{1}\left(S^{3}-L ; \mathbb{Z}\right) \cong H_{2}\left(B^{4}, S^{3}-L ; \mathbb{Z}\right) \cong H^{2}\left(B^{4}, L ; \mathbb{Z}\right) \cong$ $H^{2}\left(X^{*}, 2\right.$-handles; $\left.\mathbb{Z}\right) \rightarrow H^{2}\left(X^{*} ; \mathbb{Z}\right)$ induced by $\partial, P D$, excision and inclusion, respectively. Since the latter group is also free abelian, $h[\gamma]$ is the uniquely defined class $\frac{1}{2}\left(\sum r\left(K_{i}\right) h\left[\partial D_{i}\right]+h\left[\partial z_{2}\right]\right)$. Setting $\rho_{0}=h[\gamma]$, we see from the definition of $h$ that $\rho_{0} \mid M=P D \Gamma(\xi, s)$. Similarly, $h\left[\partial D_{i}\right]=P D\left[D_{i}\right]$ in $X^{*}$, so $\left\langle h\left[\partial D_{i}\right], \alpha_{j}\right\rangle=\delta_{i j}$. Now $\partial z_{2}=\partial z_{3}$ for some cycle $z_{3}$ in $\left(B^{4}, S^{3}-L\right)$, and $h\left[\partial z_{2}\right]=P D\left[z_{3}\right]$ in $X^{*}$. Thus, $\left\langle h\left[\partial z_{2}\right], \alpha_{i}\right\rangle=\left[z_{3}\right] \cdot \alpha_{i}=\left[z_{3}-z_{2}\right] \cdot \alpha_{i}$ since $z_{2}$ lies in $M=\partial X^{*}$, and so $\left\langle\rho_{0}, \alpha_{i}\right\rangle=\frac{1}{2}\left(r\left(K_{i}\right)+\left[z_{3}-z_{2}\right] \cdot \alpha_{i}\right)$ for each $\alpha_{i}$. But $z_{3}-z_{2}$ is an integer cycle in $X^{*}$ whose image in $H_{2}\left(X^{*}, B^{4} ; \mathbb{Z}_{2}\right)$ agrees with that of $z_{1}$. Thus $\left[z_{3}-z_{2}\right]$ is an integer lift of the $\mathbb{Z}_{2}$-class represented by $L_{0}+L^{\prime}$, and the observation following the theorem shows that the given class $\rho$ satisfies $\rho\left|M=\rho_{0}\right| M=P D \Gamma(\xi, s)$.

We are now ready to define the 2-fold lift $\tilde{\Theta}(\xi, s, f)$ of $\Theta_{f}(\xi) \in \mathbb{Z} / 2 d(\xi)$, obtaining a complete set of homotopy invariants for oriented 2-plane fields.

Definition 4.15. Let $\xi$ be an oriented 2-plane field on a closed, oriented 3-manifold $M$ (not necessarily connected). Let $s$ be a spin structure on $M$, and let $f$ be a framing on $\Gamma(\xi, s) \in H_{1}(M ; \mathbb{Z})$. Choose a vector field $v$ as in Definition 4.7 determining $s$, with zero locus $2 \gamma$ carried by $L$, and the framing $f$ determined by a framing (also called $f$ ) on $L$. (For example, we can take all multiplicities of $\gamma$ to be 1 by the proof of Proposition 4.8.) Let $X$ be any compact, almost-complex 4-manifold with almostcomplex boundary $(M, \xi)$. Then $v$ determines a complex trivialization of $T X \mid M-L$. Let $z$ be a 2-cycle in $(X, L)$ that is dual to the relative Chern class $c_{1}(X, v) \in H^{2}(X, M-L ; \mathbb{Z})$. Define $\tilde{\Theta}(\xi, s, f)$ to be $Q_{f}(z)-2 \chi(X)-3 \sigma(X) \in \mathbb{Z} / 4 d(\xi)$.

Note that adding a twist to $f$ on $\Gamma(\xi, s)$ corresponds to adding 4 twists to the induced framing $\tilde{f}$ on 
$P D c_{1}(\xi)=2 \Gamma(\xi, s)$.

Theorem 4.16. Let $M, \xi, s$ and $f$ on $\Gamma(\xi, s)$ be as in Definition 4.15. Then $\tilde{\Theta}(\xi, s, f) \in \mathbb{Z} / 4 d(\xi)$ depends only on $M, s, f$ and the homotopy class of $\xi$, and it reduces to $\Theta_{\tilde{f}}(\xi) \operatorname{modulo} 2 d(\xi)$. If $\Gamma\left(\xi, s_{0}\right)=\Gamma\left(\xi, s_{1}\right)$ then $\tilde{\Theta}\left(\xi, s_{1}, f_{1}\right)=\tilde{\Theta}\left(\xi, s_{0}, f_{0}\right)+4\left(f_{1}-f_{0}\right)+2\left\langle c_{1}(\xi), \lambda\right\rangle$, where $\lambda$ is any integer lift of $\operatorname{PD} \Delta\left(s_{0}, s_{1}\right)$. In general, $\tilde{\Theta}\left(\xi, s_{1}, f_{1}\right)=\tilde{\Theta}\left(\xi, s_{0}, f_{0}\right)+Q_{f_{0}, f_{1}}\left(z_{M}\right)$, where $z_{M}$ is any integral 2-cycle in $(I \times M,\{0,1\} \times M)$ such that $\partial z_{M}$ has the form $2\left(1 \times \gamma_{1}-0 \times \gamma_{0}\right)$ with $\left[\gamma_{i}\right]=\Gamma\left(\xi, s_{i}\right)$ and $\left[\left.z_{M}\right|_{2}\right]=P D \Delta\left(s_{0}, s_{1}\right)$. For fixed $s$ and $f, \tilde{\Theta}(\xi, s, f)$ is independent of the orientation of $\xi$, reverses sign if the orientation of $M$ is reversed, and drops by 4 if $\xi$ is changed on a component of $M$ by the canonical generator of the $\mathbb{Z}$-action of Proposition 4.1 (for any fixed $\tau$ ). Performing a surgery on $S^{0}$ in $(M, \xi)$ (i.e., a 4-dimensional 1-handle addition as in Section 2) adds 2 to $\tilde{\Theta}(\xi, s, f)$, and $\tilde{\Theta}$ adds in the obvious way under disjoint union. If $\varphi: M \rightarrow M^{\prime}$ is an orientation-preserving diffeomorphism, then $\tilde{\Theta}\left(\varphi_{*} \xi, \varphi_{*} s, \varphi_{*} f\right)=\tilde{\Theta}(\xi, s, f)$.

If $\xi_{0}$ and $\xi_{1}$ are oriented 2-plane fields on a connected $M$, then they are homotopic if and only if for some (hence, any) choice of $s$ and $f, \Gamma\left(\xi_{0}, s\right)=\Gamma\left(\xi_{1}, s\right)$ and $\tilde{\Theta}\left(\xi_{0}, s, f\right)=\tilde{\Theta}\left(\xi_{1}, s, f\right)$. If $c_{1}\left(\xi_{0}\right)$ is a torsion class, then the same is true with $\theta\left(\xi_{i}\right)$ or $\Theta_{f}\left(\xi_{i}\right)$ in place of each $\tilde{\Theta}\left(\xi_{i}, s, f\right)$. If $\Gamma\left(\xi_{0}, s\right)=\Gamma\left(\xi_{1}, s\right)$, then $\theta\left(\xi_{1}\right)-\theta\left(\xi_{0}\right)$ (if defined) lifts under finite covering maps $\pi$ by multiplying by the degree of $\pi$, and similarly for $\Theta$ and $\tilde{\Theta}$ (for fixed $s$ and $f$ ).

We can compute $\tilde{\Theta}$ for a Stein boundary $M=\partial X$ in standard form, by reconstructing the setup of the proof of Theorem 4.12. Using that notation, let $z_{1}$ be any integral 2-chain in $M$ with $\partial z_{1}=$ $2 \gamma-\sum r\left(K_{i}\right) \partial D_{i}$ for some smooth 1-cycle $\gamma$. There is a unique spin structure $s$ on $M$ determined by the equation $P D \Delta\left(s, s_{0}\right)=\left[z_{1}\right] \in H_{2}\left(M, \amalg \partial D_{i} ; \mathbb{Z}_{2}\right)$, and its characteristic sublink is obtained from the sublink representing $\left[z_{1}\right] \in H_{2}\left(X^{*}, B^{4} ; \mathbb{Z}_{2}\right)$ by subtracting the sublink $L_{0}$ coming from the 1-handles of $X$. Now $\tilde{\Theta}(\xi, s, f)$ is obtained for any framing $f$ induced from $\gamma$ on $\Gamma(\xi, s)=[\gamma]$, by adding 2handles to $X$ along $(\gamma, f)$, computing the self-intersection number $Q_{f}(z)$ of $z=\sum r\left(K_{i}\right) D_{i}+z_{1}$ suitably extended over the new 2-handles, and subtracting $2 \chi(X)+3 \sigma(X)$. The difference terms $2\left\langle c_{1}(\xi), \lambda\right\rangle$ and $Q_{f_{0}, f_{1}}\left(z_{M}\right)$ in Theorem 4.16 are easily computed once we recall that for spin structures $s_{0}$ and $s_{1}$ on $\mathrm{M}$, the sublink $L_{\Delta}$ corresponding to $P D \Delta\left(s_{0}, s_{1}\right)$ is the difference of the corresponding characteristic sublinks. In fact, $2\left\langle c_{1}(\xi), \lambda\right\rangle$ is given by $2 \sum r\left(K_{i}\right) \lambda_{i}$, where $\sum \lambda_{i} \alpha_{i} \in H_{2}\left(X^{*} ; \mathbb{Z}\right)$ lifts $L_{\Delta}$ and pulls back to a class $\lambda \in H_{2}(M ; \mathbb{Z})$. To compute $Q_{f_{0}, f_{1}}\left(z_{M}\right)$, it suffices to assume that $\left(\gamma_{0}, f_{0}\right)$ is carried by a 0 -framed knot in $\partial B^{4} \subset X^{*}$, then extend $z_{M}$ by a surface in $B^{4}$ with boundary $\gamma_{0}$ and add handles along $\gamma_{1}$ as before.

Here are a few simple applications of the theorem.

Corollary 4.17. If $\xi$ is an oriented 2-plane field with $c_{1}(\xi)=0$ on a connected $M$, and $H_{1}(M ; \mathbb{Z})$ has no 2-torsion, then all orientation-preserving self-diffeomorphisms $\varphi$ of $M$ preserve $\xi$ up to homotopy.

Proof. Since $2 \Gamma(\xi, s)=P D c_{1}(\xi)=0$, we must have $\Gamma(\xi, s)=0=\Gamma\left(\varphi_{*} \xi, s\right)$ for any $s$. Since $\theta\left(\varphi_{*} \xi\right)=\theta(\xi)$, the result follows.

Corollary 4.18. Let $M$ be an integral homology 3-sphere with an orientation-reversing self-diffeomorphism $\varphi$. If $M$ bounds a smooth, orientable rational homology ball $X$ (e.g., if $M=S^{3}$ or $\Sigma \# \bar{\Sigma}$ for any homology sphere $\Sigma$ ), then no 2-plane field $\xi$ (or nowhere zero vector field) is invariant up to homotopy under $\varphi$.

Proof. The rational ball $X$ admits an almost-complex structure. (For example, the double of $X$ admits an almost-complex structure with isolated singularities $[\mathrm{HH}]$.) The resulting complex line field $\xi_{0}$ on $M$ has $\theta\left(\xi_{0}\right)=-2$. Since $H_{1}(M ; \mathbb{Z})=0$, all 2-plane fields on $M$ can be oriented and the $\mathbb{Z}$-action of Proposition 4.1 acts transitively on the resulting homotopy classes, so any plane field $\xi$ satisfies $\theta(\xi) \equiv 2$ $(\bmod 4)$. But if $\xi$ is preserved by $\varphi$ up to homotopy then $\theta(\xi)=-\theta(\xi)=0$.

Corollary 4.19. Let $M$ be a spherical space form with a (positive) contact structure $\xi_{0}$ whose universal cover is the tight structure on $S^{3}$. If $\xi_{1}$ is any other such contact structure on $M$ with $\Gamma\left(\xi_{1}, s\right)=\Gamma\left(\xi_{0}, s\right)$, then $\xi_{1}$ is homotopic to $\xi_{0}$. If $M \neq S^{3}$ then there is no negative contact structure $\xi_{2}$ on $M$ with $\Gamma\left(\xi_{2}, s\right)=$ 
$\Gamma\left(\xi_{0}, s\right)$ and tight universal cover. In particular, any negative contact structure on the Poincaré homology sphere must have an overtwisted universal cover.

Proof. Since the tight positive contact structure on $S^{3}$ is unique, we must have $\theta\left(\tilde{\xi}_{1}\right)=\theta\left(\tilde{\xi}_{0}\right)$, where $\tilde{\xi}_{i}$ is the universal cover of $\xi_{i}$. Thus $\theta\left(\xi_{1}\right)=\theta\left(\xi_{0}\right)$ and so $\xi_{0}$ and $\xi_{1}$ are homotopic. If $\xi_{2}$ exists, then it is in the same $\mathbb{Z}$-orbit as $\xi_{0}$, so $\theta\left(\tilde{\xi}_{2}\right)-\theta\left(\tilde{\xi}_{0}\right)$ must be an integer divisible by 4 times the degree of the cover. But $\theta\left(\tilde{\xi}_{0}\right)=-2$, so $\theta\left(\tilde{\xi}_{2}\right)=+2$ and the cover has degree 1 . The Poincaré homology sphere admits such a $\xi_{0}$ (since the complex line field on $\partial B^{4}$ in $\mathbb{C}^{2}$ is invariant under the binary icosahedral group in $S U(2)$ ), so it admits no negative $\xi_{2}$ with a tight universal cover.

Example 4.20. Let $X$ be the 4 -manifold obtained by attaching a 2-handle to $B^{4}$ along an oriented knot $K$ with framing 0 . The almost-complex structures on $X$ are classified by the integers, by setting $J_{k}$ equal to the unique structure with $c_{1}\left(J_{k}\right)$ given by $2 k$ times the canonical generator of $H^{2}(X ; \mathbb{Z})$. We will compute $\tilde{\Theta}$ for the 2-plane field $\xi_{k}$ induced on $M=\partial X$ by $J_{k}$. (Note that $\theta\left(\xi_{k}\right)$ is not defined unless $k=0$.) When $K$ is the unknot, $M=S^{1} \times S^{2}$, and there is a diffeomorphism $\varphi: M \rightarrow M$ fixing the $S^{1}$-coordinate and acting on the family of 2 -spheres by the nontrivial element of $\pi_{1}(S O(3))$. We will show that $\varphi$ preserves the homotopy class of $\xi_{k}$ if and only if $k$ is either odd or 0 .

There are exactly two spin structures on $M$. Let $s$ be the one that extends over $X$, and let $s^{\prime}$ be the other one. Since $H_{1}(M ; \mathbb{Z}) \cong \mathbb{Z}$ has no 2 -torsion, we must have $\Gamma\left(\xi_{k}, s\right)=\Gamma\left(\xi_{k}, s^{\prime}\right)=\frac{1}{2} P D c_{1}\left(\xi_{k}\right)=k[\partial D]$ where $D$ is a cocore of the 2 -handle. The plane field on $\partial B^{4}$ determined by $J_{k}$ contains a nowhere zero vector field, which extends to a vector field $v$ in $\xi_{k}$ with zero locus $2 k \partial D$, and $v$ induces the spin structure $s$ on $M$. We can set $z=2 k D$ in Definition 4.15. Let $f_{n}$ denote the $n$-framing on $\partial D$ (as measured in $\partial B^{4}$ ), which induces a framing on $\gamma=k \partial D$. (Note that we can also obtain framings on $\Gamma\left(\xi_{k}, s\right)=k[\partial D]$ between $f_{n}$ and $f_{n+1}$, by splitting $\gamma$ into $k$ disjoint circles.) Clearly, $Q_{f_{n}}(z)=4 k^{2} n$, so $\tilde{\Theta}\left(\xi_{k}, s, f_{n}\right)=4\left(k^{2} n-1\right) \in \mathbb{Z} / 8 k$. Since the generator $\lambda$ of $H_{2}(M ; \mathbb{Z})$ lifts $P D \Delta\left(s, s^{\prime}\right)$, changing the spin structure adds $4 k$ to $\tilde{\Theta}, \tilde{\Theta}\left(\xi_{k}, s^{\prime}, f_{n}\right)=4\left(k^{2} n+k-1\right) \in \mathbb{Z} / 8 k$. Now if $K$ is the unknot, then $\varphi$ interchanges $s$ and $s^{\prime}$, and it preserves $\partial D$, changing $f_{n-1}$ to $f_{n}$. Thus, $\tilde{\Theta}\left(\varphi_{*} \xi_{k}, s, f_{n}\right)=\tilde{\Theta}\left(\xi_{k}, s^{\prime}, f_{n-1}\right)=$ $4\left(k^{2}(n-1)+k-1\right)=\tilde{\Theta}\left(\xi_{k}, s, f_{n}\right)+4 k(1-k) \in \mathbb{Z} / 8 k$. Since $\Gamma\left(\varphi_{*} \xi_{k}, s\right)=\Gamma\left(\xi_{k}, s\right), \varphi_{*} \xi_{k}$ is homotopic to $\xi_{k}$ if and only if $4 k(1-k)$ vanishes modulo $8 k$, i.e., $k$ is either odd or 0 . As a check, observe that $\varphi \circ \varphi$ always preserves $\xi_{k}$ as it should (being isotopic to the identity). Note that for any $k$ and $n, \Theta_{f_{n}}\left(\xi_{k}\right)=-4 \in \mathbb{Z} / 4 k$, so the full invariant $\tilde{\Theta}$ is required to distinguish $\varphi_{*} \xi$ from $\xi$.

Proof of Theorem 4.16. We proceed as in the proof of Theorem 4.5. Given $\left(M, \xi_{0}\right)$ as in Definition 4.15 and $\xi_{1}$ homotopic to $\xi_{0}$, choose almost-complex manifolds $X_{0}, X_{1}$ and $W$ as before. For $i=0,1$, choose $s_{i}, f_{i}, v_{i}, \gamma_{i}, L_{i}$ and $z_{i}$ for $\left(X_{i}, \partial X_{i}\right)$ as in Definition 4.15 , and let $\tilde{\Theta}_{i} \in \mathbb{Z} / 4 d\left(\xi_{0}\right)$ denote the resulting value for $\tilde{\Theta}\left(\xi_{i}, s_{i}, f_{i}\right)$ on $\partial X_{i}= \pm M$. Note that $\partial z_{i}=2 \gamma_{i}$. Let $z_{M}$ be a cycle in $\left(I \times M, 0 \times L_{0} \cup 1 \times L_{1}\right)$ dual to the relative Chern class $c_{1}\left(I \times M, v_{0}, v_{1}\right) \in H^{2}\left(I \times M, 0 \times\left(M-L_{0}\right) \cup 1 \times\left(M-L_{1}\right) ; \mathbb{Z}\right)$ for the given almost-complex structure on $I \times M \subset W$. Then $\partial z_{M}=-2\left(0 \times \gamma_{0}+1 \times \gamma_{1}\right)$ in $\partial I \times M($ recall that $P D$ reverses sign under orientation reversal on $M)$, and the cycle $z=z_{0}+z_{M}+z_{1}$ in $W$ is dual to $c_{1}(W)$. Reducing modulo 2 , we have $\left[\left.z_{M}\right|_{2}\right]=P D w_{2}\left(I \times M, s_{0}, s_{1}\right) \in H_{2}\left(I \times M ; \mathbb{Z}_{2}\right)$, or projecting into $M,\left[\left.z_{M}\right|_{2}\right]=P D \Delta\left(s_{0}, s_{1}\right) \in H_{2}\left(M ; \mathbb{Z}_{2}\right)$. Now $c_{1}^{2}(W)=z^{2}=Q_{f_{0}}\left(z_{0}\right)+Q_{f_{0}, f_{1}}\left(z_{M}\right)+Q_{f_{1}}\left(z_{1}\right)$, so $0=c_{1}^{2}(W)-2 \chi(W)-3 \sigma(W)=\tilde{\Theta}_{0}+\tilde{\Theta}_{1}+Q_{f_{0}, f_{1}}\left(z_{M}\right)$. In the case $\Gamma\left(\xi_{0}, s_{0}\right)=\Gamma\left(\xi_{1}, s_{1}\right)$ (relative to the original orientation of $M), \gamma_{0}$ and $-\gamma_{1}$ will be homologous, so we may compute $Q_{f_{0}, f_{1}}\left(z_{M}\right)$ by writing $z_{M}$ as $2\left(I \times \gamma_{0}\right)+z^{\prime}$ (changing $z_{M}$ and $\left(\gamma_{1}, f_{1}\right)$ by attaching a framed cobordism if necessary) where $z^{\prime}$ is an integer cycle in $I \times M$ with $\left[\left.z^{\prime}\right|_{2}\right]=P D \Delta\left(s_{0}, s_{1}\right)$. Now $Q_{f_{0}, f_{1}}\left(z_{M}\right)=\hat{z}_{M}^{2}=4\left(f_{1}-f_{0}\right)+4 \gamma_{0} \cdot z^{\prime}=$ $4\left(f_{1}-f_{0}\right)+2\left\langle c_{1}\left(\xi_{0}\right), z^{\prime}\right\rangle$. Setting $f_{0}=f_{1}$ and $s_{0}=s_{1}$, we see that $\left[z^{\prime}\right] \in H_{2}(M ; \mathbb{Z})$ is even, so that $Q_{f_{0}, f_{1}}\left(z_{M}\right) \equiv 0 \bmod 4 d\left(\xi_{0}\right)$, and $\tilde{\Theta}_{1}=-\tilde{\Theta}_{0}$. Thus, $\tilde{\Theta}(\xi, s, f)$ depends only on $M, s, f$ and the homotopy class of $\xi$, and it flips sign when the orientation of $M$ is reversed. In the general case, we now have $\tilde{\Theta}\left(\xi_{0}, s_{1}, f_{1}\right)=-\tilde{\Theta}_{1}=\tilde{\Theta}\left(\xi_{0}, s_{0}, f_{0}\right)+Q_{f_{0}, f_{1}}\left(z_{M}\right)$, and the formula remains true if we replace $z_{M}$ by any other relative cycle $z_{M}^{\prime}$ with the same boundary and with $\left[\left.z_{M}^{\prime}\right|_{2}\right]=P D \Delta\left(s_{0}, s_{1}\right)$ (since $z_{M}^{\prime}-z_{M}$ will be an integral cycle in $M$ with $\left[z_{M}^{\prime}-z_{M}\right] \in H_{2}(M ; \mathbb{Z})$ even). It is immediate from the definitions that $\tilde{\Theta}(\xi, s, f)$ reduces $\bmod 2 d(\xi)$ to $\Theta_{\tilde{f}}(\xi)$, and the rest of the first paragraph of Theorem 4.16 is also clear, except for the assertion about the $\mathbb{Z}$-action. 
To compute the effect of the standard generator $g$ of the $\mathbb{Z}$-action in Proposition 4.1 on a component of $M$ (adding a right twist to the framing of $\Gamma_{\tau}(\xi)$ ), we fix a trivialization $\tau$ of $T M$ and a 2-plane field $\xi$, and form $X$ as before. Let $\xi^{\prime}$ be a plane field on $M$ obtained from $\xi$ via $g$. We can assume that $\xi=\xi^{\prime}$ outside of a ball $B$ in $M$, that $\varphi_{\xi}^{-1}(p) \cap B=\emptyset$ and that $\varphi_{\xi^{\prime}}^{-1}(p) \cap B$ is an unknot with framing 1 . Now we alter the almost-complex structure on $X$ in a neighborhood of $B$ so that it has a unique singularity and its field of complex lines on $M$ is $\xi^{\prime}$. We can remove the singularity by a suitable sum with copies of $S^{2} \times S^{2}$ as in the proof of Lemma 4.4, and use the new manifold $X^{\prime}$ to compute $\tilde{\Theta}\left(\xi^{\prime}, s, f\right)$. Clearly, this construction is local, so the change in $\tilde{\Theta}$ is independent of our initial choices, including $M, \xi, X$ and $\tau$, and $\theta$ (when defined) changes by the same number. It is obvious from the Hirzebruch-Hopf formula (see the proof of Lemma 4.4) that the change must be divisible by 4, but to determine it exactly we compute it in an example. Let $M=S^{3}$ and let $\xi$ and $\xi^{\prime}$ be the unique negative and positive tight contact structures on $M$. Then $\theta\left(\xi^{\prime}\right)-\theta(\xi)=-4$, and we verify that $\xi^{\prime}$ is obtained from $\xi$ via $g$ (up to homotopy). Let $S^{3}$ be the unit sphere in the quaternions $\mathbb{H}=\{q=z+w j \mid z, w \in \mathbb{C}\}$. The complex structure is given by left multiplication by $i$, and $S^{3}=S U(2)$ acts by right multiplication, determining a canonical trivialization $\tau$ of $T M$. The standard contact structure $\xi^{\prime}$ is orthogonal to the right-invariant vector field $v^{\prime}(q)=i q$, which corresponds to the constant map $\varphi_{\xi^{\prime}}(q)=i$ (into $S^{2} \subset \operatorname{Im} \mathbb{H}$ ) under $\tau$. The opposite structure $\xi$ is orthogonal to a vector field $v$ obtained from $v^{\prime}$ by conjugating by the orientation-reversing map $q \mapsto \bar{q}=\bar{z}-w j$. Thus, $v(q)=-q i$ and $\varphi_{\xi}(q)=-q i \bar{q}$. Clearly, $\varphi_{\xi}(q)=\varphi_{\xi}\left(q^{\prime}\right)$ if and only if $q$ and $q^{\prime}$ lie in the same orbit under right multiplication by $S^{1}=\mathbb{C} \cap S^{3}$. Since left multiplication by $S^{1}$ induces the standard Hopf fibration, whose fibers have linking number +1 , the fibers of $\varphi_{\xi}$ will have linking number -1 . Thus, $g$ sends $\xi$ to $\xi^{\prime}$, as required.

We have now verified that $g$ lowers the invariants $\theta \in \mathbb{Q}$ and $\tilde{\Theta} \in \mathbb{Z} / 4 d(\xi)$ by 4 . By Proposition 4.1, $g$ generates a $\mathbb{Z}$-action on each set $\Gamma_{\tau}^{-1}(x)$ of a connected $M$, and $\Gamma_{\tau}^{-1}(x)$ is isomorphic to $\mathbb{Z} / d(\xi)$ as a $\mathbb{Z}$-space. Thus, $\tilde{\Theta}$ (or $\theta$ when defined) distinguishes any two nonhomotopic plane fields with the same value of $\Gamma_{\tau}=\Gamma(\cdot, s)$, so $\Gamma$ and $\tilde{\Theta}$ (or $\theta$ ) are a complete set of invariants for distinguishing homotopy classes of plane fields. Furthermore, for a degree $k$ covering $\widetilde{M} \rightarrow M$ and the trivialization of $T \widetilde{M}$ lifting that of $T M, g$ will lift to $k g$ on $\widetilde{M}$, so differences in $\tilde{\Theta}$ or $\theta$ on $\Gamma_{\tau}^{-1}(x)$ will multiply by $k$ under lifting.

\section{Contact 3-manifolds.}

We now examine holomorphically fillable contact structures on oriented 3-manifolds in more detail. We show that many such structures have finite covers that are overtwisted (Proposition 5.1). As an example, we exhibit a pair of fillable structures on an oriented 3-manifold that are are homotopic as plane fields (Example 5.2), and distinguish them by observing that under a certain 2-fold cover one becomes overtwisted while the other remains tight. We then expand our notation to allow rational surgeries on links (Proposition 5.3 and surrounding text) and proceed to exhibit fillable structures on several families of oriented 3-manifolds. We show that "most" oriented Seifert fibered spaces admit fillable positive contact structures (Theorem 5.4). For example, all such spaces that fiber over (possibly nonorientable) surfaces other than $S^{2}$ bound Stein surfaces realizing both boundary orientations, as do many Brieskorn spheres, and all Seifert fibered spaces bound Stein surfaces after possibly reversing orientation (Corollary 5.5). Our construction frequently yields contact structures representing more than one homotopy class of 2-plane fields on the manifold; we examine the case of circle bundles over surfaces in detail (Corollary 5.7). For a family of examples most of which are hyperbolic, we realize "most" rational surgeries on the Borromean rings as oriented boundaries of Stein surfaces (Theorem 5.9 and subsequent corollaries). For an arbitrary link, Proposition 5.8 discusses the local topology of the set of $n$-tuples of rational coefficients for which surgery yields a Stein boundary.

Proposition 5.1. Let $(M, \xi)$ be a contact 3-manifold exhibited as the boundary of a Stein surface in standard form (Definition 2.1). Suppose that the diagram intersects some disk in $\mathbb{R}^{2}$ in a collection of $m \geq 1$ parallel strands in the configuration shown in Figure 28, and let $\gamma$ be a loop surrounding the strands as shown. Let $(\widetilde{M}, \tilde{\xi}) \rightarrow(M, \xi)$ be any locally contactomorphic covering map such that some conjugate of $\gamma$ in $\pi_{1}(M)$ is not in the image of $\pi_{1}(\widetilde{M})$. Then $\tilde{\xi}$ is an overtwisted contact structure.

For example, if $L$ is any Legendrian link in $\# n S^{1} \times S^{2}$, and we modify $L$ by adding zig-zags to some component $K$ to decrease $t b(K)$ by 2 without changing $r(K)$, then the proposition applies to the manifold 


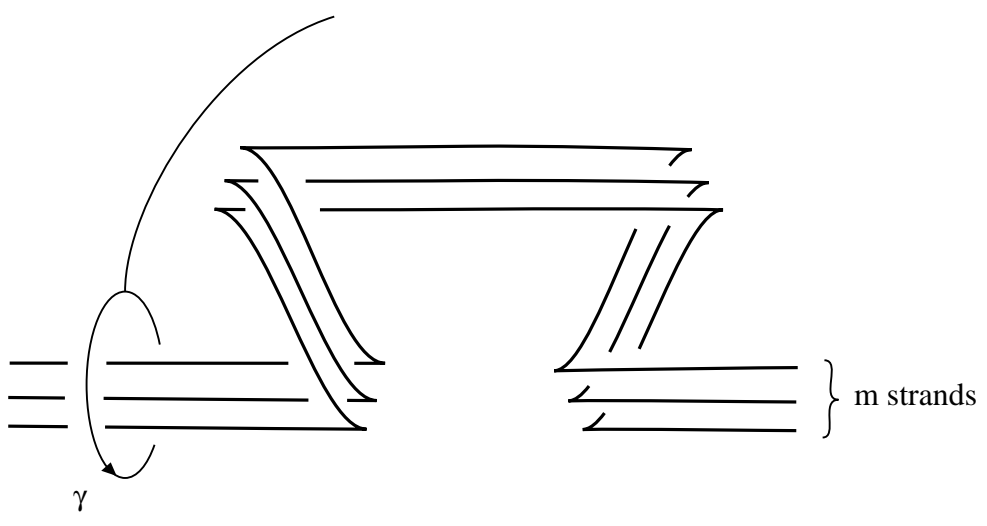

FiguRE 28

$(M, \xi)$ obtained by contact surgery on the modified link, with $\gamma$ a meridian of $K$. Applying this to the unknot in $S^{3}$, we see that any lens space of the form $L(p, 1), p \geq 4$, admits fillable structures all of whose covers are overtwisted. Of course, these same lens spaces are pseudoconvex boundaries of negative holomorphic disk bundles over $S^{2}$, and all covers of the resulting contact structures are tight, since they are fillable by holomorphic disk bundles (by branched covering).

Proof. Let $C$ be the Legendrian curve shown in Figure 29(a), with $t b(C)=-2$. As a smooth knot in the complement of the given link, $C$ is isotopic to the Whitehead double of $\gamma$, so it bounds an immersed disk $D$ disjoint from the link, as is clearly visible in Figure 29(b). The framing induced by $D$ on $C=\partial D$ is the blackboard framing in Figure 29(b), corresponding to the writhe $w(C)=-2=t b(C)$. Thus, $D$ induces the canonical framing on $C$. The $\pi_{1}$-condition on $\gamma$ guarantees that some lift $\widetilde{D}$ of $D$ is an embedded disk in $\widetilde{M}$. Since $\widetilde{D}$ still induces the canonical framing on its boundary, it is an overtwisted disk in $\widetilde{M}$, i.e., its boundary is an unknot with $t b(\partial \widetilde{D})=0$.

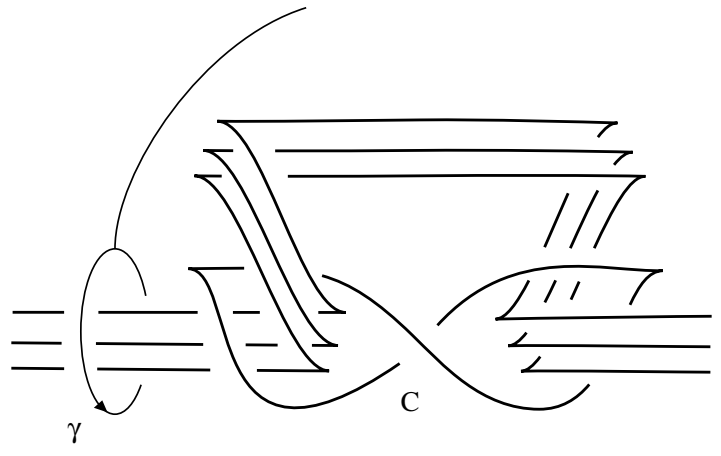

(a)

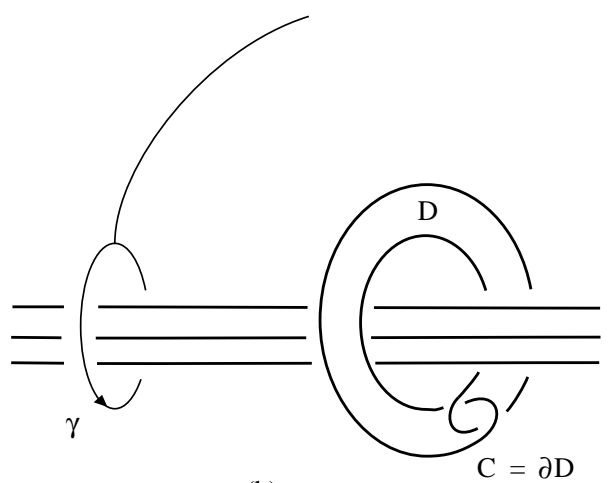

(b)

FigURE 29

Example 5.2. Let $(M, \xi)$ be the boundary of the Stein surface $X$ obtained by attaching a 2-handle to the Legendrian knot $K \subset S^{1} \times S^{2}$ shown in Figure 30(a). Then $t b(K)=1$ and $r(K)=0$, so the framing on $K$ is 0 and $c_{1}(X)=0$. By changing the 1-handle to a 2-handle (cf. Figure 26), we see that $M$ is also obtained by 0 -surgery on the symmetric link $L$ shown in Figure $30(\mathrm{~b})$. Since $H_{1}(M ; \mathbb{Z}) \cong \mathbb{Z} \oplus \mathbb{Z}$ has no 2-torsion and $c_{1}(X)=0$, we have $\Gamma(\xi, s)=0$ for all spin structures $s$, and $\theta(\xi)$ is defined and equals -2 . Since the link $L$ is symmetric, there is an orientation-preserving diffeomorphism $\varphi: M \rightarrow M$ that interchanges the two surgery tori. Clearly, the fillable positive contact structure $\varphi_{*} \xi$ on $M$ has the same invariants $\Gamma$ and $\theta$ as $\xi$, so by Theorem 4.16, $\xi$ and $\varphi_{*} \xi$ are homotopic as 2-plane fields. To 
distinguish the two contact structures, we pass to a double cover. Since $\pi_{1}(X) \cong \mathbb{Z}, X$ has a unique double cover $\widetilde{X}$. The corresponding contact manifold $(\widetilde{M}, \tilde{\xi})$ covering $(M, \xi)$ bounds $\tilde{X}$, so it is tight. However, the lift of $\varphi_{*} \xi$ to $\widetilde{M}$ is contactomorphic to a double cover of $(M, \xi)$ with multiplicity 2 along $K$, so Proposition 5.1 shows that it is overtwisted. Thus, $\xi$ and $\varphi_{*} \xi$ cannot be isotopic, although they are both contactomorphic (via $\varphi$ ) and homotopic as 2-plane fields. For similar examples that are not homotopic, consider Example 4.14 with $p \geq 2$. These examples can also be distinguished by a new technique of Akbulut and Matveyev [AM] relying on gauge theory.

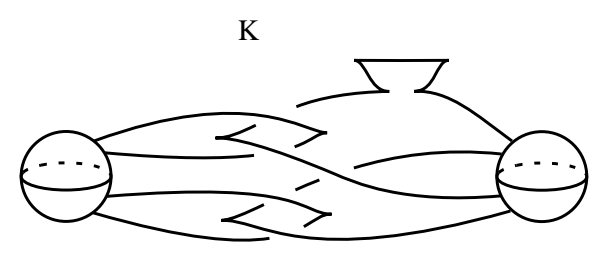

(a)

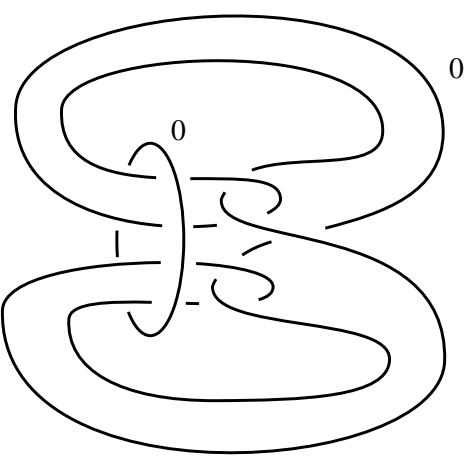

(b)

FiguRE 30

For the remainder of the paper, we will work with rational surgery diagrams for 3-manifolds. For more details, see [GS] or [Ro]. Let $M_{0}$ be an oriented 3-manifold, and suppose we have established a convention for determining 0 -framings of knots in $M_{0}$. In our applications, $M_{0}$ will usually be $\# n S^{1} \times S^{2}$ $(n \geq 0)$ exhibited in standard form as in Section 2. Let $L$ be a link in $M_{0}$. If we assign a rational number $r_{i}=\frac{p_{i}}{q_{i}} \in \mathbb{Q} \cup\{\infty\}\left(p_{i}, q_{i}\right.$ coprime integers $)$ to each component $K_{i}$ of $L$, then we obtain a 3-manifold $M$ by rational surgery on $L$ with coefficients $r_{i}$, as follows. Each $K_{i}$ has a tubular neighborhood $\nu K_{i}$. Let $\left(\mu_{i}, \lambda_{i}\right)$ be a positively oriented basis for $H_{1}\left(\partial \nu K_{i} ; \mathbb{Z}\right) \cong \mathbb{Z} \oplus \mathbb{Z}$, where $\lambda_{i}$ is determined up to sign as the class of a parallel copy of $K_{i}$ determined by the 0 -framing, and $\mu_{i}$ is determined by a suitably oriented meridian (nullhomologous circle in $\nu K_{i}$ ); see Figure 31. We obtain $M$ by cutting each $\nu K_{i}$ out of $M_{0}$ and regluing it by a diffeomorphism of $\partial \nu K_{i}$ sending $\mu_{i}$ to $p_{i} \mu_{i}+q_{i} \lambda_{i}$. This procedure determines $M$ up to orientation-preserving diffeomorphism. Thus, setting $r_{i}=\infty$ corresponds to deleting $K_{i}$ from $L$. If $M_{0}=\partial(0$-handle $\cup 1$-handles $)$ in standard form and all coefficients are integral, then $M$ is the boundary of the 4-manifold obtained by attaching handles along $L$ using the same coefficients. For this $M_{0}$, any 1 -handle can be replaced by a 0 -framed 2 -handle without changing $M$; recall Figure 26 . Two other useful moves leaving $M$ unchanged are the Rolfsen twist and slam-dunk. For $m \in \mathbb{Z}$, an $m$-fold Rolfsen twist (Figure 32) is obtained by cutting $M_{0}$ open along a disk bounded by an unknotted component $K_{i}$ of $L$, and regluing it with $m$ right $360^{\circ}$ twists. (A negative number denotes $|m|$ left twists.) The reciprocal of the coefficient of $K_{i}$ increases by $m$ as shown, and the other coefficients $r_{j}$ increase by $m\left(\ell k\left(K_{i}, K_{j}\right)\right)^{2}$. A slam-dunk (Figure 33) is obtained from a pair of components $K_{i}, K_{j}$ of $L$ with $K_{j}$ a meridian of $K_{i}$ and $r_{i} \in \mathbb{Z}$, by pushing $K_{j}$ across $\partial \nu K_{i}$ into the solid torus glued to $\partial \nu K_{i}$, changing the coefficient of $K_{i}$ to $r_{i}-\frac{1}{r_{j}}$.

Proposition 5.3. Let $L$ be a Legendrian link in standard form in $\# n S^{1} \times S^{2}$, with a rational coefficient $r_{i}$ assigned to each component $K_{i}$. If $r_{i}<t b\left(K_{i}\right)$ or $r_{i}=\infty$ for each $i$, then the manifold $M$ obtained by rational surgery on $L$ with these coefficients is the oriented boundary of a Stein surface.

Proof. First, we erase each component of $L$ with coefficient $\infty$, which does not change $M$. For each 


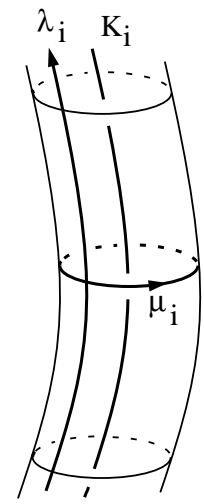

FiguRE 31
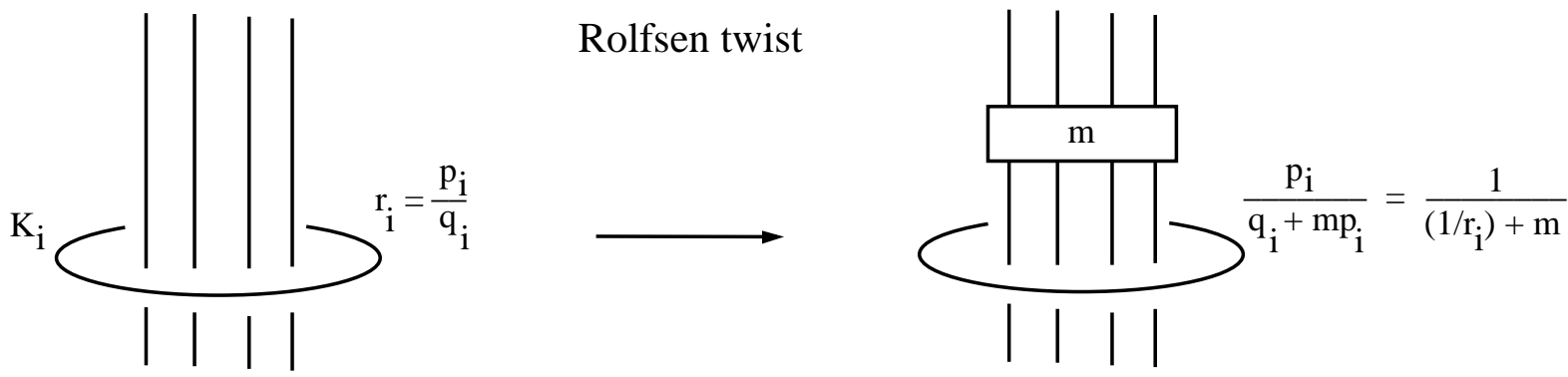

Figure 32

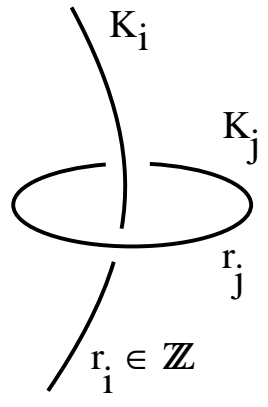

\section{slam-dunk}

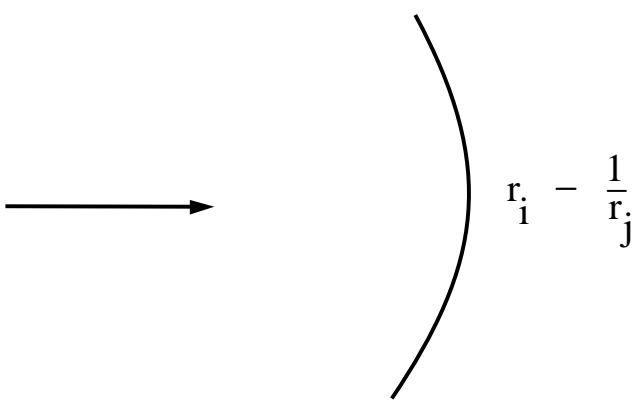

FiguRE 33

remaining component $K_{i}$, the coefficient $r_{i} \in \mathbb{Q}$ has a unique continued fraction expansion of the form

$$
r_{i}=a_{0}-\frac{1}{a_{1}-\frac{1}{a_{2}-\cdots-\frac{1}{a_{k}}}},
$$

with each $a_{j} \in \mathbb{Z}$, and $a_{j} \leq-2$ for $j \neq 0$. In fact, $a_{0}$ is the greatest integer $\leq r_{i}$, and the remaining coefficients can be obtained by successively solving and taking the greatest integer part. Modify $L$ by adding a chain of $k$ unknots linked to $K_{i}$ and associating integer coefficients $a_{0}, \ldots, a_{k}$ as shown in Figure 34. This well-known procedure leaves $M$ unchanged, as is easily seen by a sequence of slamdunks. Since $a_{0} \leq t b\left(K_{i}\right)-1$ and $a_{j} \leq-2$ for $j \neq 0$, we can add zig-zags until $t b-1$ for each component 
equals the given integer. Applying this procedure to each component $K_{i}$ of $L$, we obtain a diagram of a Stein surface whose oriented boundary is $M$.

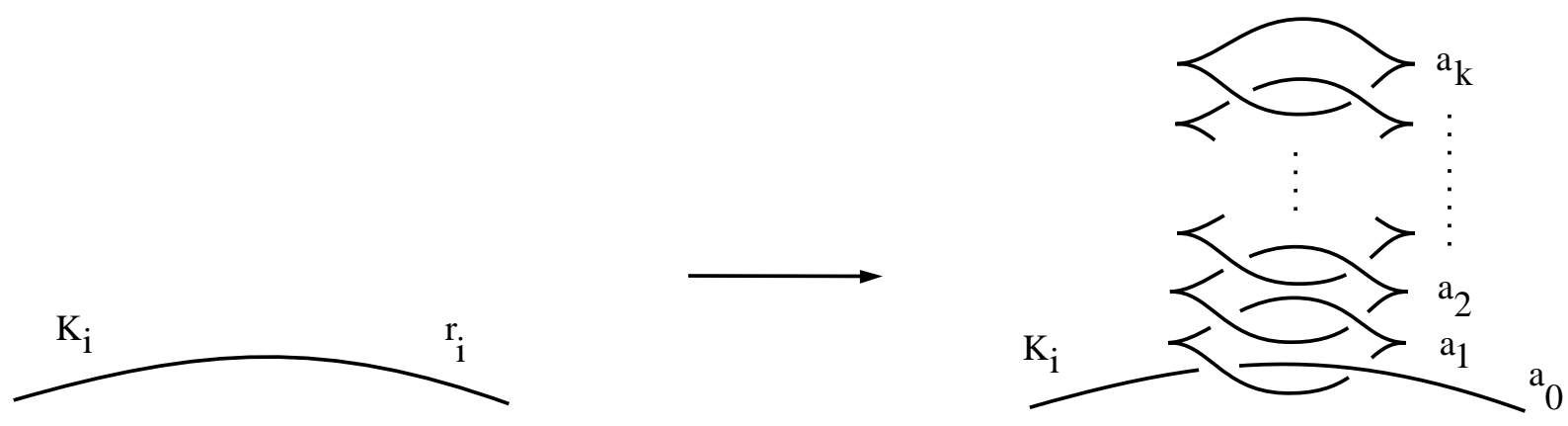

FiguRE 34

Now we can use diagrams in standard form with rational coefficients $r_{i}<t b\left(K_{i}\right)$ to draw Stein surfaces bounded by the corresponding 3 -manifolds obtained by rational surgery. To uniquely specify a Stein surface, we can use Figure 34 and an additional convention: Fix an orientation on $L$, orient the new link components so that the linking numbers in Figure 34 are nonnegative, and require all additional zig-zags to be oriented upward, decreasing $r(K)$. In general, the resulting Stein structure depends on the choice of orientation of $L$, and other Stein structures can be obtained by allowing zig-zags oriented in both directions. In practice, we will usually disregard the nonuniqueness when realizing 3-manifolds as Stein boundaries. For a simple example of this construction, observe that any lens space $L(p, q)$ is obtained by $-\frac{p}{q}$-surgery on the unknot, with $-\frac{p}{q}<-1$ (except for $S^{3}$ and $S^{1} \times S^{2}$ ). The proposition realizes any $L(p, q)$ as the oriented boundary of a Stein surface, usually with a variety of Stein structures. Thus, we see the construction more clearly with contact lenses.

For a deeper example, we consider oriented Seifert fibered spaces. These are oriented, connected 3manifolds $M$ that are foliated by circles, so that the quotient is a closed, not necessarily orientable, surface $F$, and the fibers can be described locally (over orientable neighborhoods in $F$ ) as the orbits of a circle action. The manifold $M$ is obtained from the circle bundle over $F$ with oriented total space and vanishing Euler class $\left(F \times S^{1}\right.$ when $f$ is orientable) by rational surgery on $k \geq 0$ fibers (whose 0 -framings are determined by the projection to $F$ ) with coefficients $r_{i}=\frac{p_{i}}{q_{i}}, p_{i}$ a positive integer called the multiplicity, $i=1, \ldots, k$. The pairs $\left(p_{i}, q_{i}\right)$, together with data determining $F$, are called the (unnormalized) Seifert invariants, and specify $M$ up to orientation- and fiber-preserving diffeomorphism. We obtain $\bar{M}(=M$ with its opposite orientation) by reversing the sign of each $r_{i}$. The Euler number $e(M)=\sum_{i=1}^{k}-\frac{1}{r_{i}} \in \mathbb{Q}$ is an invariant of the Seifert fibration that reverses sign under orientation reversal. Neumann and Raymond ([NR], Corollary 5.3) showed that for $F$ orientable and $e(M)<0, M$ is the oriented, strictly pseudoconvex boundary of a compact complex surface (a negative definite plumbing), hence, of a Stein surface [Bo]. We show that Seifert manifolds are oriented Stein boundaries under much weaker hypotheses. For $r \in \mathbb{Q}$, let $r=\llbracket r \rrbracket+\operatorname{frac}(r)$ denote the decomposition of $r$ with $\llbracket r \rrbracket \in \mathbb{Z}$ and $\operatorname{frac}(r) \in[0,1)$. Then $-\llbracket-r \rrbracket$ is the smallest integer $\geq r$, so $\llbracket r \rrbracket+\llbracket-r \rrbracket=-1$ for $r \notin \mathbb{Z}$. For $F$ orientable, let $e_{0}(M)$ denote $\sum_{i=1}^{k} \llbracket-\frac{1}{r_{i}} \rrbracket \in \mathbb{Z}$, which is also an invariant of the Seifert fibration. Clearly, $e_{0}(M)+e_{0}(\bar{M})=-k_{0}$, where $k_{0}$ is the number of coefficients with $\frac{1}{r_{i}} \notin \mathbb{Z}$. (Note that a circle bundle over an oriented base is given by the single coefficient $r=-\frac{1}{e}$, where $e$ is the Euler number, and so $e_{0}(M)=e$. The integer $b$ arising in the normalized form of the invariants must then be $e_{0}(M)$ or $-e_{0}(\bar{M})$, depending on one's normalization convention.)

Theorem 5.4. Let $M$ be an oriented Seifert fibered space with possibly nonorientable base $F$. If $F \approx S^{2}$, define $r_{i}^{\prime} \in[-\infty,-1), i=1, \ldots, k$, by $-\frac{1}{r_{i}^{\prime}}=\operatorname{frac}\left(-\frac{1}{r_{i}}\right)$, with $r_{i}$ as above. Then $M$ is the oriented boundary of a compact Stein surface if at least one of the following conditions is satisfied:

a) $F \not S^{2}$ 
b) $F \approx S^{2}$ and $e_{0}(M) \neq-1$

c) $F \approx S^{2}$ and if $k \geq 3$ there is a permutation of the indices of $r_{1}^{\prime}, \ldots, r_{k}^{\prime}$ after which $r_{i}^{\prime}<n\left(r_{1}^{\prime}, r_{2}^{\prime}\right)$ for all $i \geq 3$, where the function $n$ is defined below. This last condition is automatically satisfied if $k \leq 2$ or $r_{i}^{\prime}<-2$ for all $i$ or $r_{i}^{\prime}<-\llbracket \frac{1}{\left(1 / r_{1}^{\prime}\right)+1} \rrbracket-1$ for all $i \geq 2$.

Corollary 5.5. (a) Any oriented Seifert fibered space $M$ bounds a compact Stein surface after possibly reversing orientation.

(b) An oriented Seifert fibered space $M$ bounds compact Stein surfaces realizing both orientations, except possibly when $F \approx S^{2}$ and $e_{0}(M)$ equals -1 or $1-k_{0}$.

(c) The following Brieskorn homology spheres bound compact Stein surfaces realizing both orientations, except possibly for the negative orientation on $\Sigma(2,3,5)$, the Poincaré homology sphere: $\Sigma\left(p_{1}, p_{2}, m p_{1} p_{2} \pm\right.$ $1), \Sigma\left(2, p_{2},(2 m+1) p_{2} \pm 2\right), \Sigma\left(2, p_{2}, p_{3}\right)$ with $p_{2}<11, \Sigma\left(3, p_{2}, p_{3}\right)$ with $p_{2}<7$.

Part (c) of the corollary is included as a test case of condition (c) of the theorem, since Brieskorn spheres with three multiple fibers always fail conditions (a) and (b) for one choice of orientation. (See the remark following the proof of the corollary.) The author has encountered only one stubborn Seifert fibered homology sphere (cf. Corollary 4.19).

Conjecture 5.6. Only one orientation on the Poincaré homology sphere $\Sigma(2,3,5)$ is realized as the boundary of a Stein surface.

We define the function $n: Q \times Q \rightarrow \mathbb{Z} \cup\{\infty\}$ of Theorem 5.4, with $Q=[-\infty,-1) \cap(\{-\infty\} \cup \mathbb{Q})$, as follows. (Note that $-\infty=\infty$; the signs are chosen for compatibility with the ordering.) Given $r_{1}^{\prime}, r_{2}^{\prime} \in Q$, define $s \in(-\infty,-1]$ by $\frac{1}{s}=-1-\frac{1}{r_{1}^{\prime}}$. If $s=r_{2}^{\prime}$, set $n\left(r_{1}^{\prime}, r_{2}^{\prime}\right)=0$ (meaning that $M$ is a Stein boundary regardless of $\left.r_{3}^{\prime}, \ldots, r_{k}^{\prime}\right)$. Otherwise, there is a projective linear transformation $A= \pm\left[\begin{array}{ll}a & b \\ c & d\end{array}\right] \in P S L(2, \mathbb{Z})$ acting on $\mathbb{Q} \cup\{\infty\}$ (slopes in $\mathbb{Z} \times \mathbb{Z}$ ) by $A r=\frac{c+d r}{a+b r}$, such that $A s \in(-1,0]$ and $A r_{2}^{\prime} \in[-\infty,-1$ ). For example, there is a unique such $A$ with $A s=0$. Let

$$
t=\left\{\begin{array}{ll}
0 & \text { if } A 0 \in[0, \infty] \\
\frac{1}{A s} & \text { if } A 0 \in[-1,0) \\
A r_{2}^{\prime} & \text { if } A 0 \in(-\infty,-1)
\end{array},\right.
$$

so that $t \in[-\infty, 0]$, and let $M=\max (|a|,|c|), m=\min (|a|,|c|)$. Let

$$
n_{A}\left(r_{1}^{\prime}, r_{2}^{\prime}\right)=-m(\llbracket t \rrbracket+1)-M .
$$

(Here, $-\llbracket-\infty \rrbracket=+\infty$.) We define $n\left(r_{1}^{\prime}, r_{2}^{\prime}\right)$ to be the supremum of $n_{A}\left(r_{1}^{\prime}, r_{2}^{\prime}\right)$ over all such $A$. For example, suppose there is an integer $\ell$ with $r_{2}^{\prime}<\ell<s$. For the largest such $\ell$, we have $\ell+1=-\llbracket-s \rrbracket=$ $-\llbracket \frac{1}{\left(1 / r_{1}^{\prime}\right)+1} \rrbracket \leq-1$. Then for $A=\left[\begin{array}{cc}1 & 0 \\ -\ell-1 & 1\end{array}\right]$ we have $A s=-(\ell+1-s)=-\operatorname{frac}(-s) \in(-1,0]$ and $A r_{2}^{\prime}=r_{2}^{\prime}-\ell-1 \in[-\infty,-1)$ as required. Thus, $n_{A}\left(r_{1}^{\prime}, r_{2}^{\prime}\right)$ is defined and equals $\ell$. We conclude that condition (c) of the theorem is satisfied whenever $r_{i}^{\prime}<\ell=-\llbracket \frac{1}{\left(1 / r_{1}^{\prime}\right)+1} \rrbracket-1$ for all $i \geq 2$. The case $r_{i}^{\prime}<-2$ for all $i$ is precisely the case $\ell=-2$, so we have verified the last sentence of the theorem.

Proof of Corollary 5.5. To prove (a), it suffices to assume that $F=S^{2}$. Then $F \times S^{1}$ is obtained by 0 -surgery on an unknot $K$, with fibers $\left\{x_{i}\right\} \times S^{1}$ corresponding to meridians of $K$, so a surgery diagram of $M$ is given by Figure 35. We can assume that either $k_{0}=k$ or $k=1$, by slam-dunking meridians with $\frac{1}{r_{i}} \in \mathbb{Z}$ and restoring the resulting integer framing on $K$ to 0 by a Rolfsen twist on a remaining meridian. But $M$ is a Stein boundary unless $k \geq 3$, in which case $e_{0}(M)+e_{0}(\bar{M})=-k_{0} \leq-3$, so either $M$ or $\bar{M}$ satisfies $e_{0} \neq-1$.

Since (b) follows immediately from Theorem 5.4, we proceed to part (c). The Brieskorn homology sphere $M=\Sigma\left(p_{1}, p_{2}, p_{3}\right)$ is defined for any pairwise coprime integers $p_{1}, p_{2}, p_{3} \geq 2$ and is the unique (up to orientation) Seifert fibered homology sphere with multiplicities $p_{1}, p_{2}, p_{3}$. The base $F$ must be $S^{2}$, and the denominators of the coefficients $r_{i}=\frac{p_{i}}{q_{i}}$ can be chosen arbitrarily subject to the constraint 


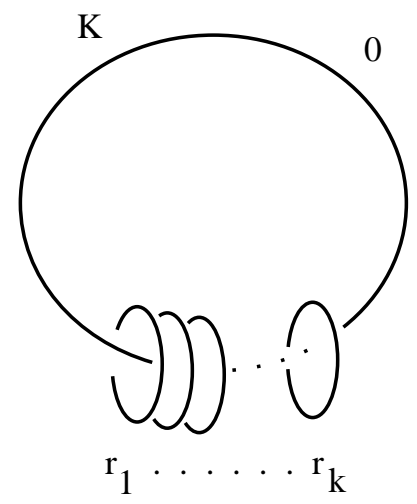

FigURE 35

that $c=q_{1} p_{2} p_{3}+p_{1} q_{2} p_{3}+p_{1} p_{2} q_{3}$ should equal \pm 1 . Clearly, $c=-p_{1} p_{2} p_{3} e(M)$, so the case $c=1$ corresponds to the usual orientation with $e(M)<0$, when $M$ is oriented as the link of the singularity of $z_{1}^{p_{1}}+z_{2}^{p_{2}}+z_{3}^{p_{3}}=0$ in $\mathbb{C}^{3}$, and $c=-1$ corresponds to the opposite orientation. (See [NR] for details.) Since $\frac{1}{r_{i}}$ is never an integer, we have $e_{0}(M)+e_{0}(\bar{M})=-3$. For each of the Brieskorn spheres listed in the corollary, we will exhibit coefficients $r_{1}, r_{2}, r_{3}$ for which $e_{0}(M)=-1$. Then $e_{0}(\bar{M})=-2$, so $\bar{M}$ is a Stein boundary and it suffices to analyze $M$, which we will do via part (c) of the theorem.

We begin with the family $\Sigma\left(p_{1}, p_{2}, m p_{1} p_{2} \pm 1\right)$. Since $p_{1}$ and $p_{2}$ are coprime, we can find $\ell_{1}, \ell_{2} \in \mathbb{Z}$ with $\left|\begin{array}{ll}p_{1} & \ell_{1} \\ p_{2} & \ell_{2}\end{array}\right|=1$. We choose $\ell_{1} \in\left(0, p_{1}\right)$, and it follows that $\ell_{2} \in\left(0, p_{2}\right)$. Let $r_{1}=\frac{p_{2}}{\ell_{2}}, r_{2}=\frac{p_{1}}{-\ell_{1}}$ and $r_{3}=\frac{m p_{1} p_{2} \pm 1}{-m}$. Clearly, the resulting $M$ satisfies $c= \pm 1$ and $e_{0}(M)=-1$, so it is $\Sigma\left(p_{1}, p_{2}, m p_{1} p_{2} \pm 1\right)$

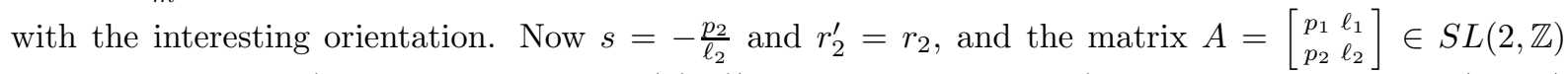
satisfies $A s=0, A r_{2}^{\prime}=-\infty$. We have $n_{A}\left(r_{1}^{\prime}, r_{2}^{\prime}\right)=-p_{1}-p_{2}>r_{3}=r_{3}^{\prime}$ except in the case of $\Sigma(2,3,5)$ oriented with positive Euler number $(c=-1)$.

Table 1 lists some other families of Brieskorn spheres by their multiplicities $p_{1}, p_{2}, p_{3}$, together with coefficients $r_{1}, r_{2}, r_{3}$ realizing them with orientation such that $e_{0}(M)=-1$, and a reason why each family consists of Stein boundaries. The signs are to be chosen consistently throughout each example, and we require $\ell, m$ and the signs to be chosen to avoid the previous examples $\Sigma\left(p_{1}, p_{2}, m p_{1} p_{2} \pm 1\right)$. All multiplicities $p_{i}$ must be $\geq 2$, but note that $m=0$ is sometimes allowed. Most of these Brieskorn spheres are Stein boundaries by the last sentence of Theorem 5.4; for the rest we list a suitable matrix $A$ with $r_{3}^{\prime}<n_{A}\left(r_{1}^{\prime}, r_{2}^{\prime}\right)$. It is easily verified that the list contains all of the remaining homology spheres in the corollary. 
TABLE 1

\begin{tabular}{|c|c|c|}
\hline$p_{1}, p_{2}, p_{3}$ & $r_{1}, r_{2}, r_{3}$ & Reason \\
\hline $2,4 \ell \pm 1,2(4 \ell \pm 1) m+4 \ell \mp 1$ & $\frac{2}{1}, \frac{4 \ell \pm 1}{-\ell}, \frac{2(4 \ell \pm 1) m+4 \ell \mp 1}{-(2 \ell \pm 1) m-\ell}$ & $r_{1}^{\prime}=-2, r_{2}^{\prime}, r_{3}^{\prime}<-3$ \\
\hline $2,4 \ell \pm 3,2(4 \ell \pm 3) m+4 \ell \pm 1$ & $\frac{2}{1}, \frac{4 \ell \pm 3}{-\ell \mp 1}, \frac{2(4 \ell \pm 3) m+4 \ell \pm 1}{-(2 \ell \pm 1) m-\ell}$ & $r_{1}^{\prime}=-2, r_{2}^{\prime}, r_{3}^{\prime}<-3$ \\
\hline $2,7,14 m \pm 3$ & $\frac{2}{1}, \frac{14 m \pm 3}{-5 m \mp 1}, \frac{7}{-1}$ & $A=\left[\begin{array}{ll}3 & 1 \\
2 & 1\end{array}\right]$ \\
\hline $2,9,18 m \pm 5$ & $\frac{2}{1}, \frac{18 m \pm 5}{-7 m \mp 2}, \frac{9}{-1}$ & $A=\left[\begin{array}{ll}3 & 1 \\
2 & 1\end{array}\right]$ \\
\hline $3,4,12 m \pm 5$ & $\frac{3}{2}, \frac{4}{-1}, \frac{12 m \pm 5}{-5 m \mp 2}$ & All $r_{i}^{\prime}<-2$ \\
\hline $3,5,15 m \pm 2$ & $\frac{3}{-1}, \frac{5}{-1}, \frac{15 m \pm 2}{8 m \pm 1}$ & All $r_{i}^{\prime}<-2$ \\
\hline $3,5,15 m \pm 4$ & $\frac{3}{2}, \frac{5}{-2}, \frac{15 m \pm 4}{-4 m \mp 1}$ & All $r_{i}^{\prime}<-2$ \\
\hline $3,5,15 m \pm 7$ & $\frac{3}{1}, \frac{5}{-1}, \frac{15 m \pm 7}{-2 m \mp 1}$ & $r_{1}^{\prime}=-\frac{3}{2}, r_{2}^{\prime}, r_{3}^{\prime}<-4$ \\
\hline
\end{tabular}

Remark. Brieskorn spheres with three multiple fibers are relatively difficult to analyze, in the sense that any such $M$ fails both conditions (a) and (b) of Theorem 5.4 for one choice of orientation. To see this, note that in general we are free to change each $\frac{1}{r_{i}}$ by adding any $m_{i} \in \mathbb{Z}$, provided that $\sum_{i=1}^{k} m_{i}=0$. (Simply Rolfsen twist along the meridians in Figure 35.) Thus, for $M=\Sigma\left(p_{1}, p_{2}, p_{3}\right)$ we may assume that $-\frac{1}{r_{1}},-\frac{1}{r_{2}} \in(0,1)$. But the equation $c= \pm 1$ shows that the numbers $q_{i}$, hence $-\frac{1}{r_{i}}$, cannot all have the same sign. Thus $-\frac{1}{r_{3}}<0$ and $e_{0}(M) \leq-1$. Similarly, $e_{0}(\bar{M}) \leq-1$. Since $e_{0}(M)+e_{0}(\bar{M})=-3$, either $e_{0}(M)$ or $e_{0}(\bar{M})$ equals -1 . In contrast, the Brieskorn sphere $M=\Sigma(2,3,5,7)$ is given by $r_{i}=\frac{2}{-1}, \frac{3}{1}, \frac{5}{3}, \frac{7}{-3}$, so $e_{0}(M)=e_{0}(\bar{M})=-2$ and both $M$ and $\bar{M}$ are Stein boundaries by Theorem $5.4(\mathrm{~b})$.

Proof of Theorem 5.4. First, we draw a link diagram of a general Seifert fibered space $M$. Figure 36(a) shows a disk bundle over a torus $T^{2}$ with Euler number $e$, drawn with two 1-handles and a 2-handle. The dashed lines are circles lifted from $\{x\} \times S^{1}$ and $S^{1} \times\{x\}$ in $T^{2}=S^{1} \times S^{1}$, and the fiber circles in the boundary of the bundle are meridians of the 2-handle. (See [GS], for example.) We put the diagram in standard form by moving a 1-handle as indicated in (a), obtaining a Legendrian knot with $t b=0$ (b). This realizes the 4 -manifold by a Stein surface, provided that $e \leq-1$. We can do a bit better (c) using the trick of Figure 20, realizing $T^{2} \times D^{2}(e=0)$. Now recall that Figure 35 shows an arbitrary Seifert fibered space with base $F=S^{2}$. If $F=T^{2}$, we obtain the corresponding picture (of an oriented, not necessarily contact, 3 -manifold) by adding meridians to Figure $36(e=0)$ with coefficients $r_{1}, \ldots, r_{k}$. If $F$ is an orientable surface of genus $g$, the picture is obtained from Figure 35 by summing $K$ with $g$ copies of Figure 37(a) and retaining the 0-framing on $K$. If $F$ is nonorientable with genus $g$, we use $g$ copies of Figure 37(b) and the 0 -framing. (In the nonorientable case, the ratio $\frac{p_{i}}{q_{i}}$ of the Seifert invariants no longer equals the surgery coefficient $r_{i}$ when the coefficient on $K$ is 0 , since the 0 -framing on $K$ no longer corresponds to $e=0$ in the circle bundle over $F$, but rather $e=-2 g$ [GS]. In fact, we have $e(M)=\sum_{i=1}^{k}-\frac{q_{i}}{p_{i}}=-2 g+\sum_{i=1}^{k}-\frac{1}{r_{i}}$.)

To begin constructing the required Stein surfaces, we first observe that we can change each $\frac{1}{r_{i}}$ by any integer using a Rolfsen twist, at the expense of changing the framing of $K$. We use this to replace each $r_{i}$ by the unique $r_{i}^{\prime}$ for which $r_{i}^{\prime} \in[-\infty,-1)$, which is given by $-\frac{1}{r_{i}^{\prime}}=\operatorname{frac}\left(-\frac{1}{r_{i}}\right)$. The resulting coefficient 


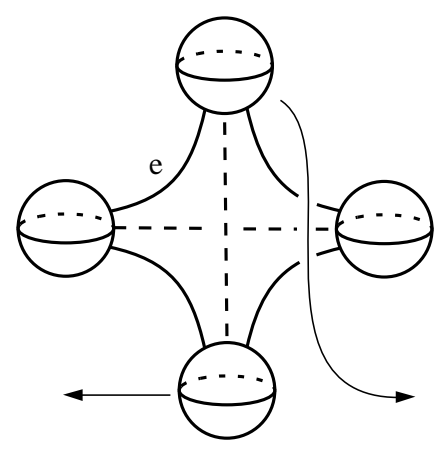

(a)

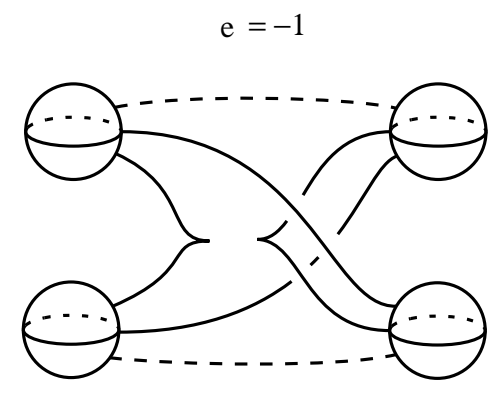

(b)

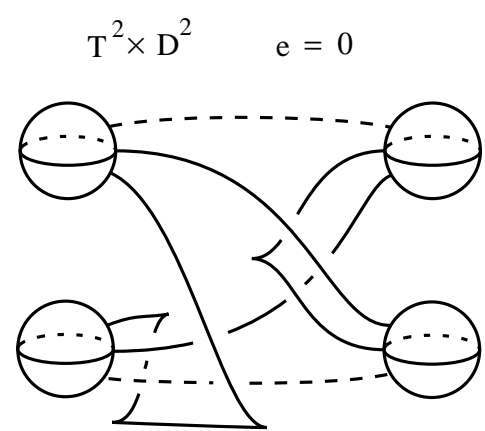

(c)

FiguRe 36

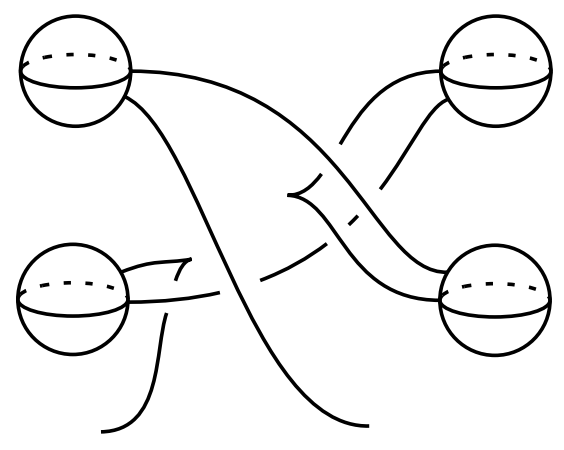

(a)

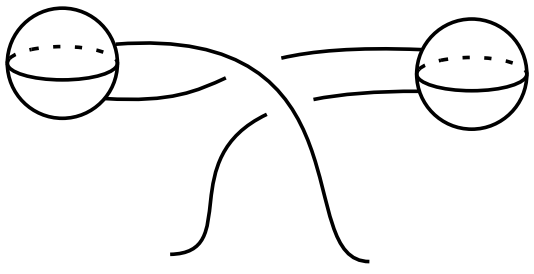

(b)

\section{FigURE 37}

on $K$ is $e_{0}(M)=\sum_{i=1}^{k} \llbracket-\frac{1}{r_{i}} \rrbracket$. (For $F$ nonorientable, we are defining $e_{0}(M)$ here.) Since we can draw $K$ as Legendrian with $t b(K)=2 g-1$ and add Legendrian meridians with $t b=-1$, Proposition 5.3 realizes $M$ as a Stein boundary, provided that $e_{0}(M)<2 g-1$. (The same technique also shows that we can realize any disk bundle over $F$ with Euler class $e \leq-\chi(F)$ by a Stein surface, provided that we improve Figure 37(b) using Figure 20.)

We next realize $M$ as a Stein boundary whenever $e_{0}(M) \geq 1$, completing the proof of part (a). We change the framing on $K$ from $e_{0}(M)$ to 1 by an inverse slam-dunk, adding a new meridian with coefficient $r_{k+1}^{\prime}=\frac{1}{1-e_{0}(M)} \in[-\infty, 0)$. For each $T^{2}$ - or $\mathbb{R} P^{2}$-summand of $F$, we surger a 1-handle to a 0 -framed 2-handle, replacing Figure 37 in the construction by Figure 38. Now $K$ is a 1-framed unknot in $S^{3}$, and we stack the other curves along it as in Figure 39. Note that as these curves pass through the disk bounded by $K$, they all lie on one or two ribbons that appear in the diagram with a right half-twist. Next, we blow down $K$ (Rolfsen twist on it with $m=-1$ ), replacing it by a full left twist as in Figure 40 . The half-twists on the ribbons become left-handed. The framing on each curve $K_{i}$ drops by $\left(\ell k\left(K, K_{i}\right)\right)^{2}$, so the new framing on each meridian is $r_{i}^{\prime}-1 \in[-\infty,-1)$. The framings on the other curves are either 0 or -4 , depending on whether $F$ is orientable ((a) or (b) in the figures). Proposition 5.3 shows that Figure 40 represents a Stein surface.

It now remains to deal with the case $F \approx S^{2}$ (Figure 35), $e_{0}(M)=-1$ or 0 . If $e_{0}(M)=0$, we can replace $K$ by a 1-handle as in Figure 41, obtaining a Stein surface. This completes the proof of part (b).

To prove part (c), it now suffices to assume that $e_{0}(M)=-1$. We can assume that $k \geq 2$, by adding coefficients $r_{i}^{\prime}=-\infty$ if necessary. After permuting the indices of $r_{1}^{\prime}, \ldots, r_{k}^{\prime}$ if desired, we slam-dunk the meridian with coefficient $r_{1}^{\prime}$ as in Figure 42. We denote the new coefficient of $K$ by $\frac{1}{s}=-1-\frac{1}{r_{1}^{\prime}}$ and the meridian labelled $r_{i}^{\prime}$ by $K_{i}, i=2, \ldots, k$. Let $T$ denote the boundary of a tubular neighborhood of 


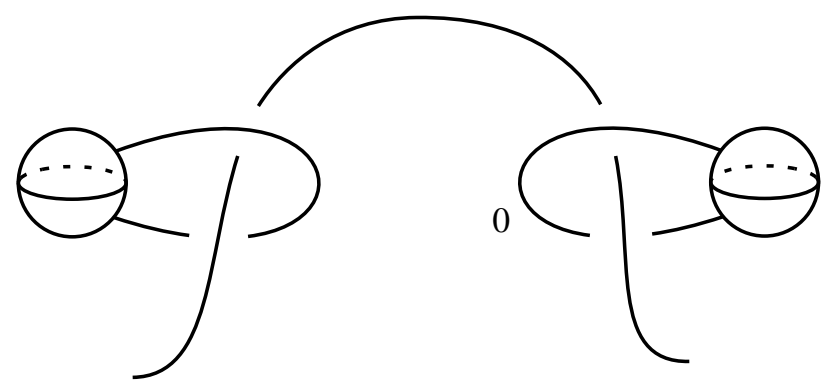

(a)

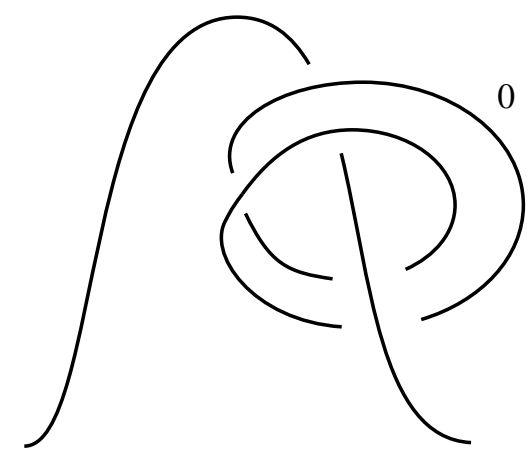

(b)

FiguRE 38

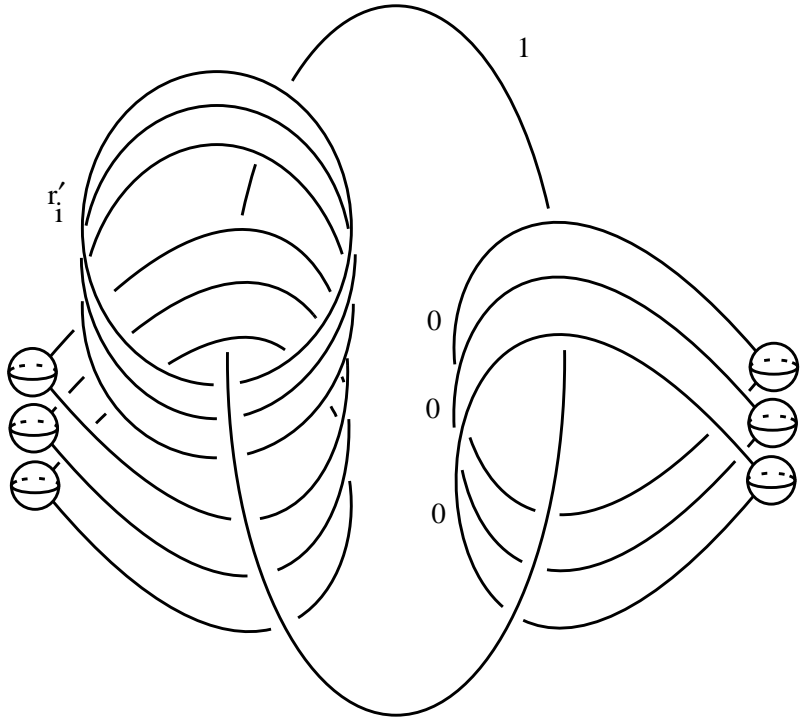

(a)

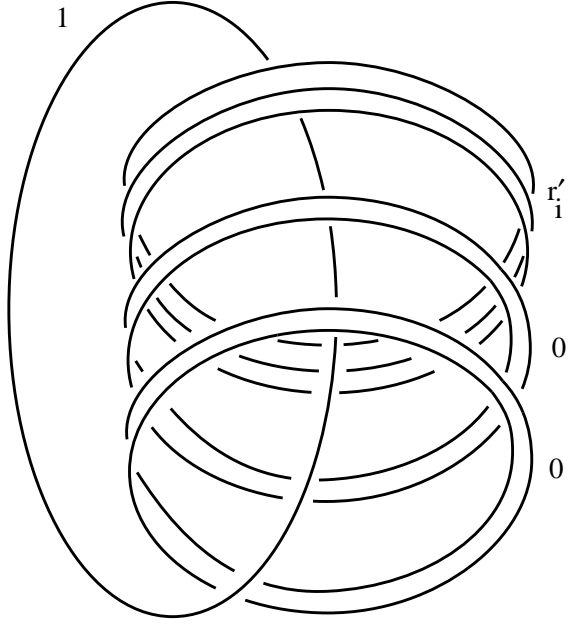

(b)

FigURE 39

$K$, containing $K_{3}, \ldots, K_{k}$ and identified with $\mathbb{R}^{2} / \mathbb{Z}^{2}$ so that $\mu$ and $\lambda$ correspond to the positive $x$ - and $y$-axes, respectively. Now we can identify the complement of a tubular neighborhood of $K \cup K_{2}$ in $S^{3}$ with $T \times I$. Thus, $M$ is the unique oriented manifold obtained from $T \times I$ by rational surgery with coefficients $r_{3}^{\prime}, \ldots, r_{k}^{\prime}$ on curves $K_{i}$ with slope 0 in $T=\mathbb{R}^{2} / \mathbb{Z}^{2}$, where the 0 -framing on each $K_{i}$ is determined by the product structure on $T \times I$, with solid tori $S^{1} \times D^{2}$ glued to the two boundary components so that $\{x\} \times \partial D^{2}$ maps to curves with slopes $s$ and $r_{2}^{\prime}$, respectively. We will complete the proof by changing coordinates in $T$ and then drawing a suitable link picture.

First, suppose that $s=r_{2}^{\prime}$. Then there is a matrix $A \in S L(2, \mathbb{Z})$ such that $A s=A r_{2}^{\prime}=0$ and $A 0=-\frac{p}{q}, p, q>0$. Applying the corresponding diffeomorphism to $T$, we obtain a Hopf link with coefficients $\infty$ and 0 , and the curves $K_{3}, \ldots, K_{k}$ are sent to parallel left-handed $(p, q)$ torus knots in $T$, linking the 0 -framed unknot $p$ times. We erase the $\infty$-framed unknot and replace the 0 -framed unknot by a 1-handle. The image of each $K_{i}, i \geq 3$, is now a copy of the knot $K^{\prime}$ in Figure 43, which is Legendrian with $t b\left(K^{\prime}\right)=-p q$. The framing $f$ determined on $K^{\prime}$ by the product structure of $T \times I$ has coefficient $-p q=t b\left(K^{\prime}\right)$ in Figure 43, as we can easily see by pushing a copy of $K^{\prime}$ off of $T$ and computing its linking number with $K^{\prime}$. Thus, $f$ equals the canonical framing on $K^{\prime}$. Since the images of the curves $K_{i}$ are parallel copies of $K^{\prime}$ determined by $f$, it follows that they fit together as a Legendrian link with each 


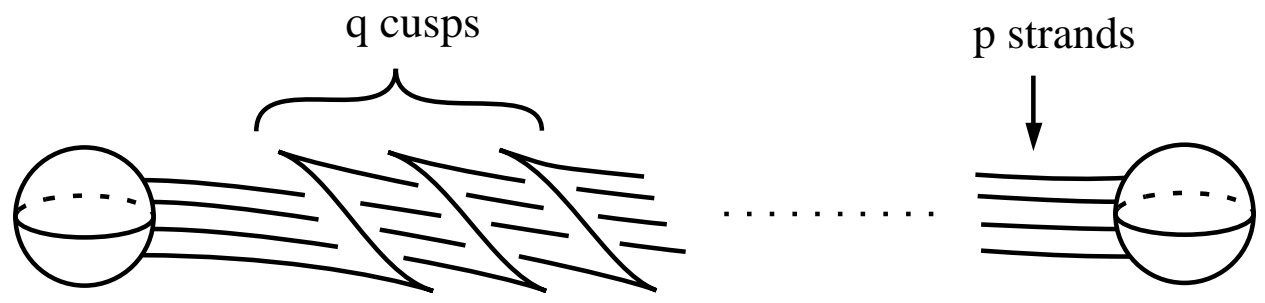

FIGURE 43

$A=\left[\begin{array}{ll}a & b \\ c & d\end{array}\right] \in S L(2, \mathbb{Z})$ such that $A s \in(-1,0]$ and $A r_{2}^{\prime} \in[-\infty,-1)$. Given any such $A$, we apply the corresponding diffeomorphism to $T$, again obtaining parallel copies of a torus knot $K^{\prime}$ in $T$ wrapped around the Hopf link, with the latter now having coefficients $\frac{1}{A s}$ and $A r_{2}^{\prime}$ in $[-\infty,-1)$. If $A 0 \in[0, \infty]$, then the torus knot is right-handed as in Figure 44. In this case $K^{\prime}$ is Legendrian with $t b\left(K^{\prime}\right)=|a c|-|a|-|c|$, and the framing $f$ induced by $T$ is $|a c|=t b\left(K^{\prime}\right)+|a|+|c|$. Since $f$ is obtained from the canonical framing by adding right twists, we can realize the images of $K_{3}, \ldots, K_{k}$ by a Legendrian link with each component satisfying $t b=t b\left(K^{\prime}\right)$, as before. The required surgery coefficients are $r_{i}^{\prime}$ relative to $f$, or $r_{i}^{\prime}+t b\left(K^{\prime}\right)+|a|+|c|$ using the standard convention. By Proposition 5.3, $M$ bounds a Stein surface in this case, provided that for $i=3, \ldots, k, r_{i}^{\prime}<-|a|-|c|=n_{A}\left(r_{1}^{\prime}, r_{2}^{\prime}\right)$.

In the remaining case, $A 0 \in(-\infty, 0)$ and the $(|a|,|c|)$ torus knot $K^{\prime}$ is left-handed. Now the picture depends on which of $|a|,|c|$ is larger, so we set $M=\max (|a|,|c|), m=\min (|a|,|c|)$ and $\left(t, t^{\prime}\right)$ equal to $\left(\frac{1}{A s}, A r_{2}^{\prime}\right)$ if $m=|c|$ and $\left(A r_{2}^{\prime}, \frac{1}{A s}\right)$ otherwise. Figure 45(a) shows the resulting Legendrian link. Since $t, t^{\prime}<-1$, the Hopf link satisfies the hypothesis of Proposition 5.3, provided that we have added no more than $-\llbracket t \rrbracket-2 \geq 0$ extra left cusps to it as shown. If $M \leq-m(\llbracket t \rrbracket+1)$, then we can draw the $(m, M)$ torus knot correctly by winding around these extra zig-zags as shown, ending as in (b) of the figure. If the inequality fails, we must add the extra $M+m(\llbracket t \rrbracket+1)$ twists to $K^{\prime}$ by the less efficient procedure on the right of the diagram, ending as in (c). In either case, $t b\left(K^{\prime}\right)=-M m-\max (0, M+m(\llbracket t \rrbracket+1))$ and the framing $f$ induced by $T$ is $-M m \geq t b\left(K^{\prime}\right)$, so we can again realize the images of $K_{3}, \ldots, K_{k}$ by a Legendrian link with $t b=t b\left(K^{\prime}\right)$ for each component. The surgery coefficients are $r_{i}^{\prime}+t b\left(K^{\prime}\right)+\max \left(0, M_{+}\right.$ $m(\llbracket t \rrbracket+1))$, and the 3 -manifold bounds a Stein surface provided that $r_{i}^{\prime}<-M-m(\llbracket t \rrbracket+1)=n_{A}\left(r_{1}^{\prime}, r_{2}^{\prime}\right)$ for $i=3, \ldots, k$. This completes the proof, since the last sentence of part (c) was verified after the definition of $n$.

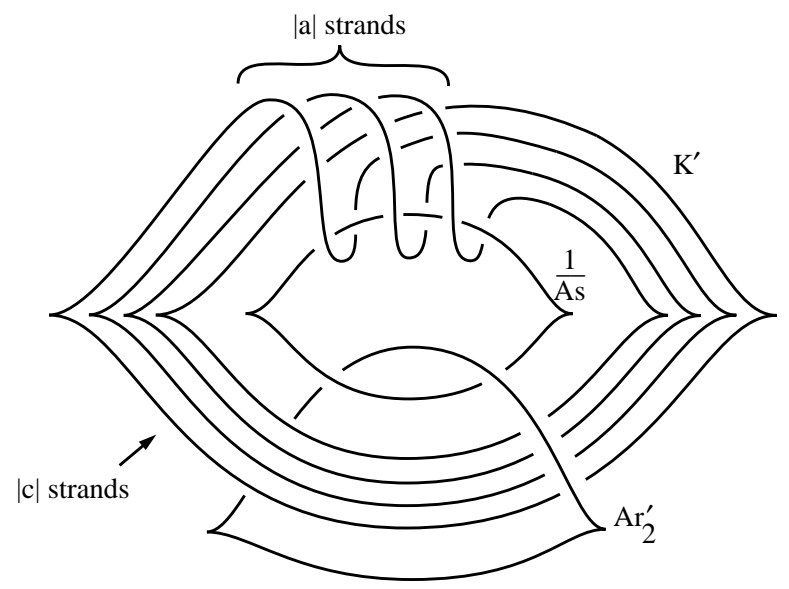

Figure 44

Note that many Seifert fibered spaces can be realized as boundaries of Stein surfaces in many ways. We have already observed that Proposition 5.3 typically produces a 4-manifold that admits Stein structures 

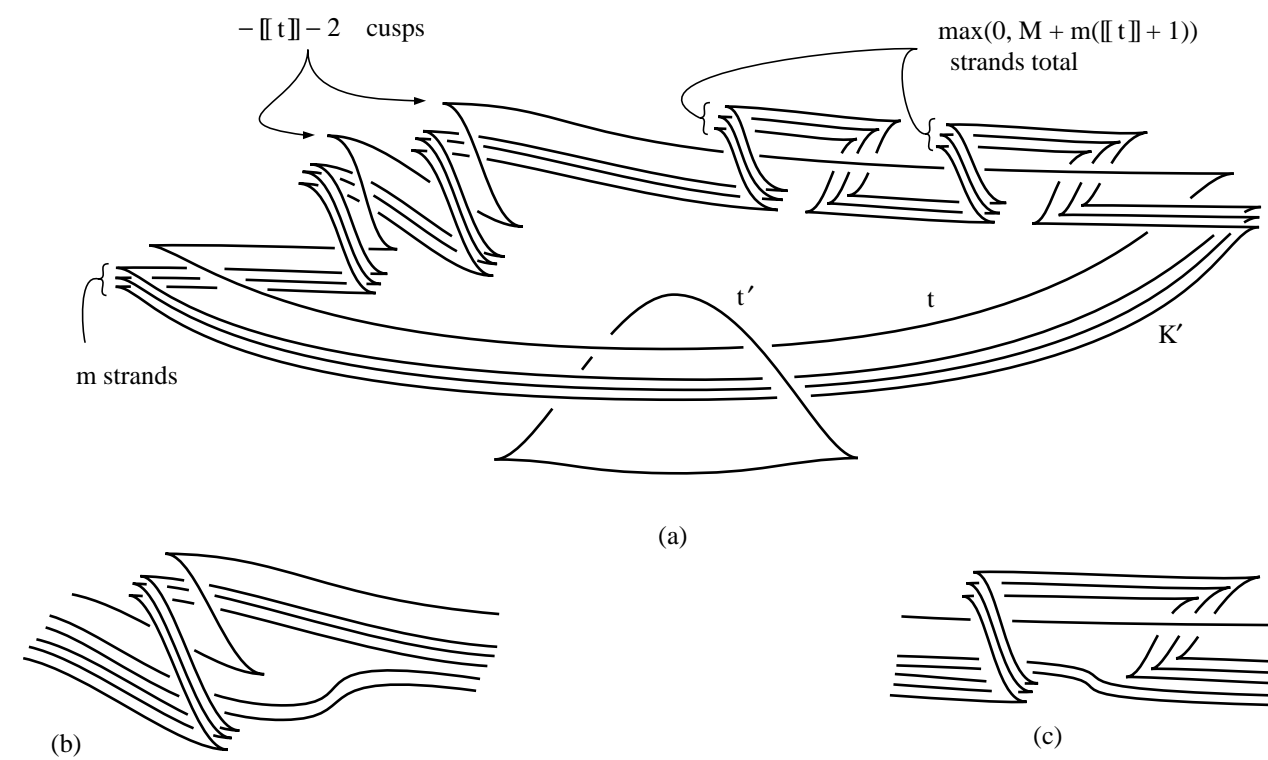

(a)

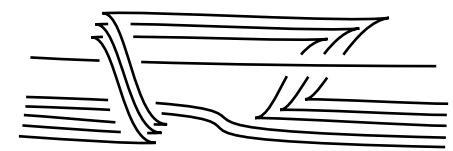

(c)

FiguRE 45

with a variety of Chern classes. In addition, the proof of Theorem 5.4 sometimes provides several methods for analyzing a given Seifert manifold - for example, if the base $F$ has genus $g>1$, then the cases of large and small $e_{0}(M)$ overlap. The author has made no systematic study of this nonuniqueness, but here is one simple family of examples. Recall that for any tight contact structure $\xi$ on a 3-manifold $M$, we have $\left|\left\langle c_{1}(\xi), F_{0}\right\rangle\right| \leq \max \left(-\chi\left(F_{0}\right), 0\right)$ for any closed, connected, oriented surface $F_{0}$ in $M$. We will call an oriented 2-plane field allowable if it satisfies this inequality for all such surfaces $F_{0}$.

Corollary 5.7. Let $M$ be the circle bundle with Euler number e over a closed, connected, oriented surface $F$ of genus $g$, and let $q \in M$. Then the oriented 3-manifold $M$ admits at least $\llbracket g-\frac{e}{2} \rrbracket$ holomorphically fillable contact structures whose homotopy classes remain distinct after allowing orientation-preserving self-diffeomorphisms of $M$. If $g \neq 0$ and $e<g$, then these contact structures (with both orientations allowed on the 2-planes) realize all homotopy classes of allowable 2-plane fields on $M-\{q\}$. For any $g$ and e, holomorphically fillable structures on $M$ and $\bar{M}$ together realize all allowable homotopy classes on $M-\{q\}$.

Proof. As in the previous proof, Figure 37(a) realizes $M$ as a Stein boundary if $e \leq 2 g-2=-\chi(F)$, via an oriented Legendrian knot $K$ in $\# 2 g S^{1} \times S^{2}$ whose meridian we denote by $\mu$. In the case of equality we have $r(K)=0$, and in general we can realize any $r(K)$ congruent to $e$ mod 2 such that $|r(K)| \leq 2 g-2-e$ (by adding zig-zags to the case $e=2 g-2$ ). By a computation similar to that of Corollary 4.6, the resulting contact structure $\xi$ has $P D c_{1}(\xi)=r(K) \mu$ and (for $\left.e \neq 0\right) \theta(\xi)=\frac{r(K)^{2}}{e}-2 \chi(F)-3$ sign $e$, so for the $\llbracket g-\frac{e}{2} \rrbracket$ different values of $|r(K)|$, the structures $\xi$ will not become homotopic after any orientationpreserving self-diffeomorphisms of $M$. To determine when these contact structures realize all allowable homotopy classes over $M-\{q\}$, recall from Corollary 4.9 that 2-plane fields $\xi$ on $M-\{q\}$ are classified by the invariant $\Gamma$, and $2 \Gamma(\xi, s)=P D c_{1}(\xi)$. If $\xi$ is an allowable plane field, then $\Gamma(\xi, s)$ pairs trivially with each of the tori in $M$ projecting to circles in $F$. Thus, $\Gamma(\xi, s)$ is a multiple $\ell \mu$ of the fiber $\mu$, and it suffices to understand which multiples we can realize. For the above contact structures, we define $X^{*}$ as in Theorem 4.12, let $s$ be the spin structure on $M$ corresponding to the characteristic sublink $K$, and compute that $\Gamma(\xi, s)=\frac{1}{2}(r(K)+e) \mu$. If $0=e<g$, we can realize $\ell \mu$ this way for any $\ell \in \mathbb{Z}$ with $2|\ell| \leq-\chi(F)$. But in this case, $F$ lifts to a section $\widetilde{F}$ of $M$, so any allowable 2-plane field satisfies $-\chi(F) \geq\left|\left\langle c_{1}(\xi), \widetilde{F}\right\rangle\right|=|2 \ell \mu \cdot \widetilde{F}|=2|\ell|$. Thus, we have finished the case $e=0$. For $e \neq 0, \mu$ has order $|e|$ in $H_{1}(M ; \mathbb{Z})$. Since the above contact structures realize $2 g-1-e$ consecutive values of $\ell$, we can realize all multiples of $\mu$ provided that $2 g-1-e \geq|e|$, or $e<g, g \neq 0$. 
To prove the final assertion, we also consider contact structures on $\bar{M}$. If $g \neq 0$ then we are done because either $M$ or $\bar{M}$ has $e \leq 0<g$. Similarly, if $g=0$, it suffices to assume $e(M)<0$. (The case $e=0$ is easy and $e(\bar{M})=-e(M)$.) But we have already realized $\Gamma(\xi, s)=\frac{1}{2}(r(K)+e) \mu$ for $r(K) \equiv e \bmod 2$, $|r(K)| \leq|e|-2$. Since $\mu$ has order $|e|$, we are only missing the case $\Gamma(\xi, s)=0$. But $\bar{M}=|e|$-surgery on the unknot is also realized by $\frac{|e|}{1-|e|}$-surgery on the unknot via a Rolfsen twist, or by a chain of $|e|-1$ unknots with framing -2 as in Figure 34, which is Stein with vanishing Chern class. Now $s$ is represented by the empty characteristic sublink (because of the Rolfsen twist) and so $\Gamma(\xi, s)=0$ as required.

For comparison, a closed, orientable 3-manifold with a taut foliation must be covered by $\mathbb{R}^{3}$ (except in the cases of $S^{1} \times S^{2}$ and $\mathbb{R} P^{3} \# \mathbb{R} P^{3}$ ), so spherical space forms such as lens spaces and the Poincaré homology sphere do not admit such foliations. In addition, many Seifert fibered spaces with universal cover $\mathbb{R}^{3}$ admit no taut foliations (or even essential laminations) [Br], although they do admit fillable contact structures, frequently with both orientations. Taut foliations on Seifert fibered spaces are typically horizontal, i.e., transverse to the fibers after a suitable isotopy (cf. [Br]). In contrast, many holomorphically fillable contact structures are neither horizontal nor vertical (parallel to the fibers after isotopy). In fact, there is a unique homotopy class of 2-plane fields transverse to a given Seifert fibration, and when $F$ is orientable any vertical 2-plane field $\xi$ has $c_{1}(\xi)=0$ (since the fibers determine a section of $\xi$ ). Thus, most of the examples in the above corollary will satisfy neither condition.

Since Seifert fibered spaces form a somewhat exceptional class of 3-manifolds, it seems useful to examine a more generic family. Every oriented 3-manifold can be realized as integer surgery on a link in $S^{3}$, so it seems natural to consider a family consisting of all integral or rational surgeries on a fixed link. We will consider the family of all rational surgeries on the Borromean rings, which includes other families such as rational surgeries on all twist knots and Whitehead links. Before proceeding with this, we observe that the set of $n$-tuples of rational coefficients for which surgery on a fixed link is a Stein boundary will be open in $(\mathbb{Q} \cup\{-\infty\})^{n}$, provided that we use the lower limit topology on $\mathbb{Q} \cup\{-\infty\}$. Note that the Stein surfaces involved may have no obvious relation to the original link.

Proposition 5.8. Let $L=K_{1} \cup \cdots \cup K_{n}$ be a link in $S^{3}$. Suppose that the oriented manifold $M$ obtained by surgery on $L$ with coefficients $r_{1}, \ldots, r_{n} \in \mathbb{Q} \cup\{-\infty\}$ bounds a Stein surface $X$. Then there are numbers $R_{i}>r_{i}$ in $\mathbb{Q}(i=1, \ldots, n)$ such that whenever $r_{i} \leq r_{i}^{\prime}<R_{i}$, the manifold $M^{\prime}$ obtained by $\left(r_{1}^{\prime}, \ldots, r_{n}^{\prime}\right)$-surgery on $L$ also bounds a Stein surface.

Proof. It suffices to assume that each coefficient $r_{i}$ is in $\mathbb{Z} \cup\{-\infty\}$, for in the general case $M$ can be expanded as an integer surgery as in the proof of Proposition 5.3, and then a small increase in $r_{i}$ can be realized by a small increase in the last coefficient $a_{k}$ of its continued fraction expansion. Write $L=L_{0} \amalg L_{\infty}$, where $K_{i} \subset L_{\infty}$ if and only if $r_{i}=-\infty$. Let $L^{\prime} \subset S^{3}-L_{0} \subset M$ denote $L_{\infty}$ union a meridian $\mu_{i}$ of each $K_{i} \subset L_{0}$. After a small perturbation, we may assume that $L^{\prime}$ is Legendrian in $(M, \xi)=\partial X$. Choose coefficients $s_{1}, \ldots, s_{n} \in \mathbb{Q} \cup\{-\infty\}$ for $L^{\prime}$, with each $s_{i}<t b\left(K_{i}^{\prime}\right)$, where $K_{i}^{\prime}=K_{i}$ or $\mu_{i}$ in $L^{\prime}$ and the 0 -framing on $K_{i}^{\prime}$ is determined via the inclusion $S^{3}-L_{0} \subset S^{3}$. By the method of proof of Proposition 5.3, we can add handles to $X$ to obtain a Stein surface whose boundary $M^{\prime}$ is obtained from $M$ by $\left(s_{1}, \ldots, s_{n}\right)$-surgery on $L^{\prime}$. By slam-dunking each $\mu_{i}$ in the original picture of $L \subset S^{3}$, we see that $M^{\prime}$ is also obtained from $S^{3}$ by surgery on $L$, with coefficients $s_{i}$ on $L_{\infty}$ and $r_{i}-\frac{1}{s_{i}}$ on $L_{0}$. Thus, we can take $R_{i}=t b\left(K_{i}\right)$ if $r_{i}=-\infty$ and $R_{i}=r_{i}+\frac{1}{\max \left(1,-t b\left(\mu_{i}\right)\right)}$ otherwise, where $t b$ is measured relative to the usual framing convention on $S^{3}$ and the contact structure $\xi$ induced by $X$.

Now let $M=M\left(r_{1}, r_{2}, r_{3}\right)$ denote the oriented 3-manifold obtained by surgery on the Borromean rings with coefficients $r_{1}, r_{2}, r_{3} \in \mathbb{Q} \cup\{\infty\}$ (Figure 46). Note that $M$ is independent of the order of the coefficients, and that reversing the orientation of $M$ is the same as reversing the sign of each $r_{i}$. Since the Borromean rings form a hyperbolic link, $M$ will be hyperbolic for "most" values of $r_{1}, r_{2}, r_{3}$.

Theorem 5.9. The oriented manifold $M\left(r_{1}, r_{2}, r_{3}\right)$ bounds a Stein surface, except possibly when $\left(r_{1}, r_{2}, r_{3}\right)$ lies in one of the subsets $A_{0}, A_{2}, A_{3}$ of $\mathbb{Q}^{3}$ defined below. Each point in $A_{j}$ has exactly $j$ negative coordinates, so $M\left(r_{1}, r_{2}, r_{3}\right)$ is always a Stein boundary if exactly one $r_{i}$ is negative.

Here, $A_{0}=\left\{\left(r_{1}, r_{2}, r_{3}\right) \mid r_{1}, r_{2}, r_{3} \in[1,4)\right\}$ and $A_{2}$ is the union of $\left\{\left(r_{1}, r_{2}, r_{3}\right) \mid r_{1} \in[0, \infty), r_{2} \in\left[-\frac{1}{3}, 0\right)\right.$, 

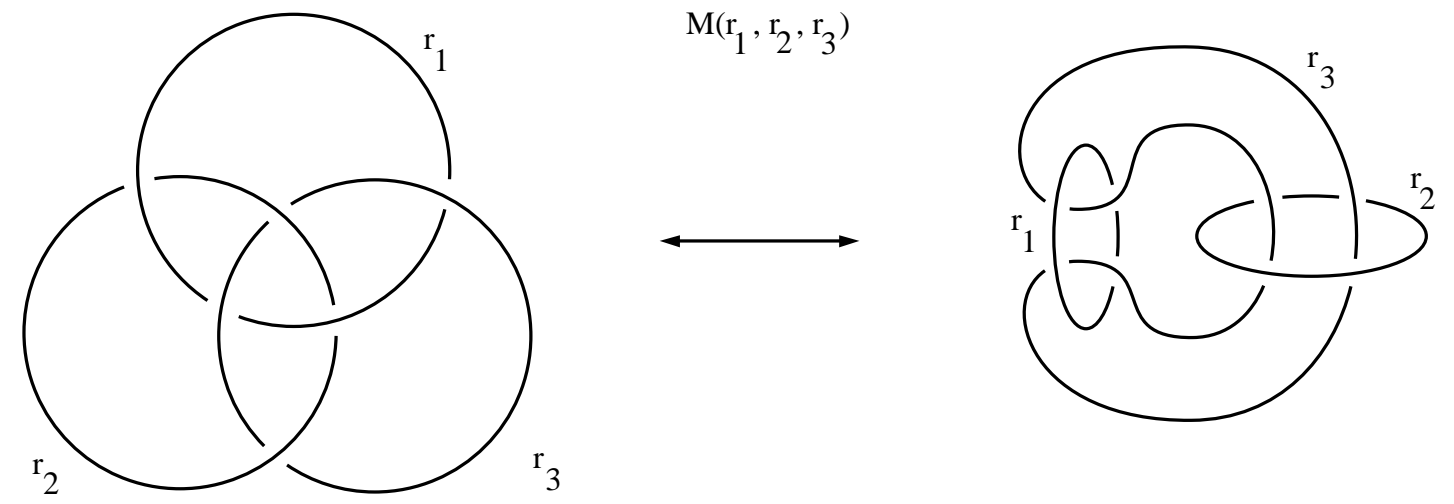

FiguRE 46

$\left.r_{3} \in\left[-2 \llbracket-\frac{1}{r_{2}} \rrbracket-1,-6\right)\right\}$ with its images under permutations of the coordinates. The set $A_{3}$ is obtained from $\left\{\left(r_{1}, r_{2}, r_{3}\right) \mid r_{1}, r_{2} \in(-\infty, 0), r_{3} \in\left[-2\left(\llbracket-\frac{1}{r_{1}} \rrbracket+\llbracket-\frac{1}{r_{2}} \rrbracket+1\right), 0\right)\right\}$ by intersecting it with its images under permutation and deleting $\left\{\left(r_{1}, r_{2}, r_{3}\right) \mid r_{1}, r_{2}, r_{3} \in[-6,0)\right.$, two $\left.r_{i} \in[-1,0)\right\}$.

Corollary 5.10. a) All integer surgeries on the Borromean rings are Stein boundaries except possibly for the finite collection whose coefficients $r_{1}, r_{2}, r_{3}$ satisfy (i) $r_{1}, r_{2}, r_{3} \in\{1,2,3\}$ or (ii) one $r_{i}$ equals -1 and the other two are in $\{-2,-3,-4\}$ or (iii) all $r_{i}=-2$.

b) All homology spheres of the form $M\left(r_{1}, r_{2}, r_{3}\right)$ are Stein boundaries except possibly if all $r_{i}=1$, when $M$ is the Poincaré homology sphere with reversed orientation.

Corollary 5.11. For fixed integers $\ell, m \in \mathbb{Z}$, let $K(\ell, m)$ be the knot shown in Figure 47, generalizing the case of a twist knot, $\ell= \pm 1$. Then all but finitely many integer surgeries on $K(\ell, m)$ are Stein boundaries. All rational surgeries on $K(\ell, m)$ are Stein boundaries provided that $\ell, m, \ell+m \leq 2$ and $\ell, m$ are not both -1 . More specifically, for $r \in \mathbb{Q} \cup\{\infty\}$, r-surgery on $K(\ell, m)$ is a Stein boundary except possibly in the following cases: (i) $\ell=m=-1,1 \leq r<4$, (ii) $\ell<0, m \geq 3,-2 m-1 \leq r<-6$ (or the same with $\ell$ and $m$ interchanged), (iii) $\ell, m>0,-2(\ell+m+1) \leq r<-6$.

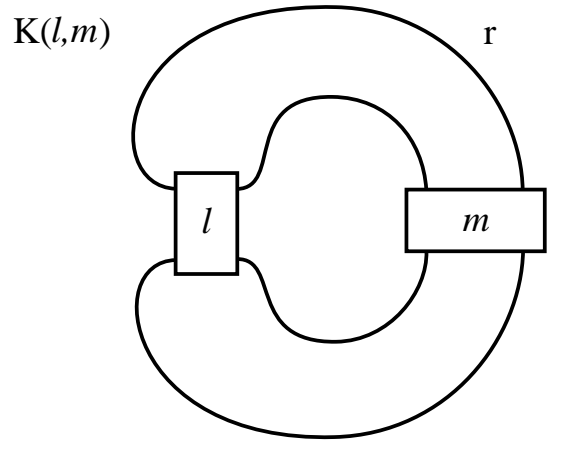

FigURE 47

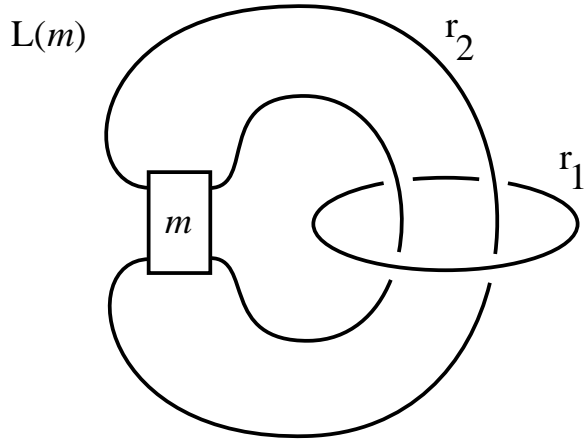

Figure 48

Corollary 5.12. For $m \in \mathbb{Z}$, let $L(m)$ denote the symmetric link in Figure 48 , generalizing the Whitehead links $m= \pm 1$. If $m \leq-2$ then all integer surgeries on $L(m)$ are Stein boundaries. If $m \leq 2$ then all but finitely many such surgeries are Stein boundaries. For any $m$ there is a finite subset $A$ of $\mathbb{Z}$ such that any surgery on $L(m)$ with coefficients in $\mathbb{Z}-A$ is a Stein boundary. Surgeries on $L(m)$ with coefficients $r_{1}, r_{2} \in \mathbb{Q} \cup\{\infty\}$ are Stein boundaries with the following possible exceptions: (i) $m=-1, r_{1}, r_{2} \in[1,4$ ), (ii) $m<0,-\frac{1}{3} \leq r_{i}<0,-2 \llbracket-\frac{1}{r_{i}} \rrbracket-1 \leq r_{j}<-6$ for $(i, j)=(1,2)$ or $(2,1)$, (iii) $m \geq 3, r_{i} \geq 0$, $-2 m-1 \leq r_{j}<-6$ for $(i, j)=(1,2)$ or $(2,1)$, (iv) $m>0, r_{1}, r_{2}<0, r_{1}$ or $r_{2}<-6$ or both $<-1$, $r_{1} \geq-2\left(\llbracket-\frac{1}{r_{2}} \rrbracket+m+1\right), r_{2} \geq-2\left(\llbracket-\frac{1}{r_{1}} \rrbracket+m+1\right)$. 
Proof of Corollaries 5.10-5.12. Corollary 5.10(a) follows immediately by looking for integer points in $A_{0} \cup A_{2} \cup A_{3}$. Part (b) follows from the observation that $H_{1}\left(M\left(\frac{p_{1}}{q_{1}}, \frac{p_{2}}{q_{2}}, \frac{p_{3}}{q_{3}}\right) ; \mathbb{Z}\right) \cong \mathbb{Z}_{p_{1}} \oplus \mathbb{Z}_{p_{2}} \oplus \mathbb{Z}_{p_{3}}$, so $M\left(r_{1}, r_{2}, r_{3}\right)$ is a homology sphere precisely when each $\frac{1}{r_{i}} \in \mathbb{Z}$. Blowing down $M(1,1,1)$ twice exhibits it as +1 -surgery on the right trefoil, which is well-known to be the Poincaré homology sphere oriented as the boundary of a positive definite $E_{8}$-plumbing. Similarly, $r$-surgery on the knot $K(\ell, m)$ is the same as $M\left(-\frac{1}{\ell},-\frac{1}{m}, r\right)$, by a pair of Rolfsen twists. Now Corollary 5.11 follows by comparing conditions (i)(iii) with the sets $A_{0}, A_{2}$ and $A_{3}$, respectively. Surgery on $L(m)$ with coefficients $r_{1}, r_{2}$ is the same as $M\left(-\frac{1}{m}, r_{1}, r_{2}\right)$, and conditions (i)-(iv) of Corollary 5.12 correspond to $A_{0}, A_{2}, A_{2}$ and $A_{3}$, respectively. (Note that when $r_{1}, r_{2} \in \mathbb{Z}$, (ii) is vacuous and (iv) implies $r_{1}, r_{2} \geq-2(m+2)$.)

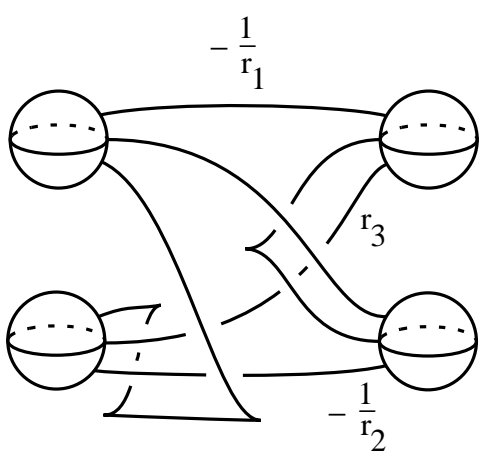

FiguRE 49

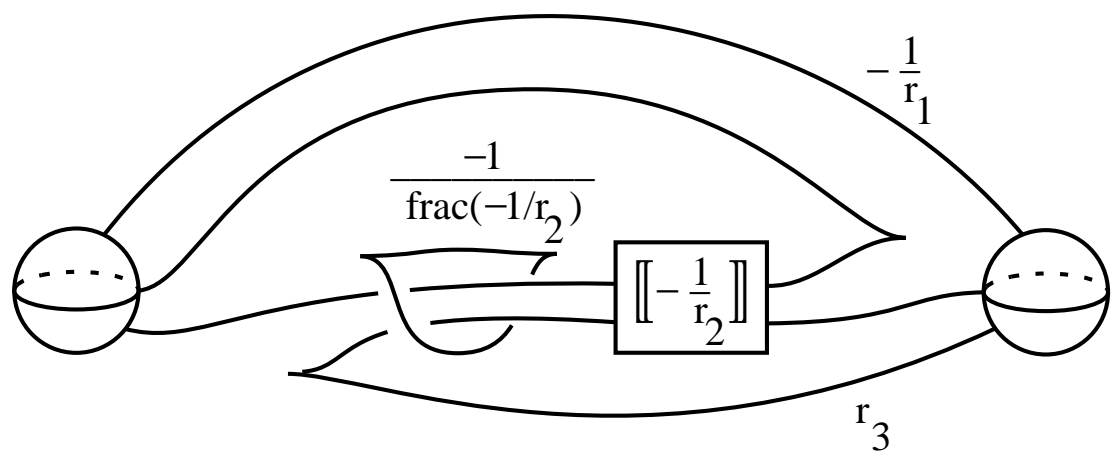

FIGURE 50

Proof of Theorem 5.9. First, we exhibit some Stein surfaces bounded by $M\left(r_{1}, r_{2}, r_{3}\right)$ for various values of the coefficients $r_{i}$. Note that if any $r_{i}=\infty$ then $M$ is a connected sum of lens spaces, hence, a Stein boundary. Figure 49 is Stein for $r_{1}, r_{2} \geq 0, r_{3}<1$, cf. Figure 36. To see that its boundary is $M\left(r_{1}, r_{2}, r_{3}\right)$, surger the 1-handles to 0-framed 2-handles and slam-dunk the meridians. Figure 50 is Stein for $r_{1} \geq 0, r_{2}<0, r_{3}<-2 \llbracket-\frac{1}{r_{2}} \rrbracket-1$ (note that the twist is right-handed), and its boundary is $M$ by a $-\llbracket-\frac{1}{r_{2}} \rrbracket$-Rolfsen twist. Similarly, Figure 51 solves the case $r_{1}, r_{2}<0, r_{3}<-2\left(\llbracket-\frac{1}{r_{1}} \rrbracket+\llbracket-\frac{1}{r_{2}} \rrbracket+1\right)$. Figure 52 is Stein when $r_{1} \geq 1, r_{2}>0$ and $r_{3} \geq 4$. (Note that the central $\left(\llbracket-\frac{1}{r_{2}} \rrbracket+1\right)$-twist makes sense, since there are $-2 \llbracket-\frac{1}{r_{2}} \rrbracket-2 \geq 0$ left cusps.) Finally, Figure 53 is Stein for $r_{1} \geq-1,-1 \leq r_{2}<0$, $r_{3} \geq-6$. To verify that Figure 52 depicts $M\left(r_{1}, r_{2}, r_{3}\right)$, apply a $-\llbracket-\frac{1}{r_{2}} \rrbracket$-Rolfsen twist to one component to change its coefficient to $r_{2}$, surger the 1-handle to a 2-handle and change its framing to $r_{3}-4$ by a slam-dunk, then blow down the -1 -framed unknot. Finally, a +1 -Rolfsen twist on one component to restore its coefficient to $r_{1}$ recovers Figure 46. (This figure arose as a generalization of the case $r_{1}=1$, $\frac{1}{r_{2}} \in \mathbb{Z}^{+}$, the twist knot $K\left(-1,-\frac{1}{r_{2}}\right)$.) To recognize Figure 53 as $M$, surger and slam-dunk to change the 1 -handle to an $\left(r_{3}+6\right)$-framed 2 -handle, +3 -Rolfsen twist to eliminate the $-\frac{1}{3}$-framed unknot, then blow down the +1 -framed unknot (which only links the $\left(r_{3}+9\right)$-framed curve). A pair of -1 -twists to recover coefficients $r_{1}$ and $r_{2}$ yields Figure 46. (Figure 53 was produced by generalizing the case $r_{1}=r_{2}=-1$, or $r_{3}$-surgery on the left trefoil, which is $\mp \sum(2,3,6 n \pm 1)$ if $r= \pm \frac{1}{n}$.)

These Stein surfaces are sufficient to prove the theorem. Given $r=\left(r_{1}, r_{2}, r_{3}\right) \in \mathbb{Q}^{3}$ with at most one $r_{i}<0$, Figure 49 or 52 will exhibit $M\left(r_{1}, r_{2}, r_{3}\right)$ as a Stein boundary unless $r \in A_{0}$. If exactly two coefficients are negative, we permute so that $r_{1} \geq 0>r_{2} \geq r_{3}$. If $r_{2}<-1$ then Figure 50 realizes $M$ as a Stein boundary, so we assume $r_{2} \in[-1,0)$. Then Figure 50 or 53 works unless $-2 \llbracket-\frac{1}{r_{2}} \rrbracket-1 \leq r_{3}<-6$. This last condition is vacuous unless $r_{2} \geq-\frac{1}{3}$, in which case $r \in A_{2}$. Finally, if all coefficients $r_{i}$ are negative, then Figure 51 or 53 will realize $M$ unless $r \in A_{3}$.

\section{REFERENCES}

[AM] S. Akbulut and R. Matveyev, A note on contact structures, Pacific Math. J. (to appear).

[Ar] V. Arnol'd, Lagrangian and Legendrian cobordisms, Funct. Anal. and Applic. 14 (1980), 167-177. 


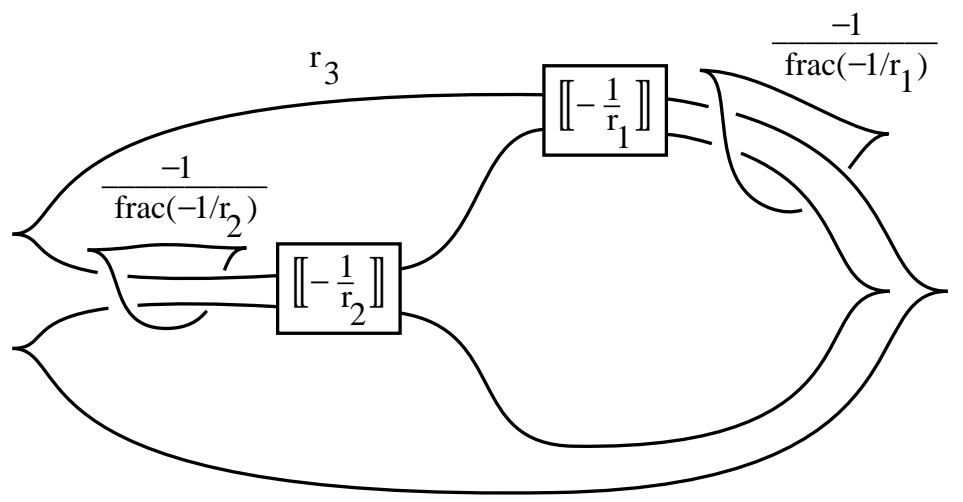

FIGURE 51

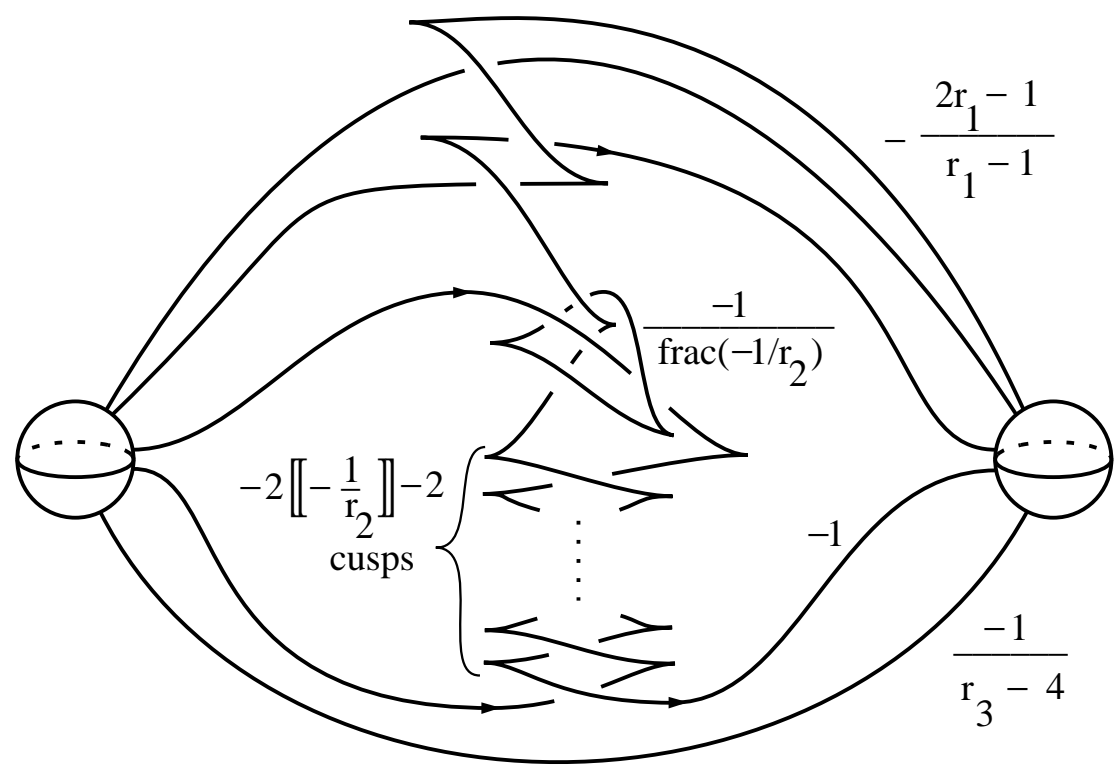

FigURE 52

[Be] D. Bennequin, Entrelacements et équations de Pfaff, Astérisque 107-108 (1983), 83-161.

[BG] Ž. Bižaca and R. Gompf, Elliptic surfaces and some simple exotic $\mathbb{R}^{4}$ 's, J. Diff. Geom. 43 (1996), $458-504$.

[Bo] Bogomolov, Fillability of contact pseudoconvex manifolds, Göttingen Univ. preprint, Heft 13 (1993), 1-13.

[Br] M. Brittenham, Essential laminations in Seifert-fibered spaces, Topology 32 (1993), 61-85.

[C] A. Casson, Three lectures on new infinite constructions in 4-dimensional manifolds, (notes prepared by L. Guillou), A la Recherche de la Topologie Perdue, Progress in Mathematics, vol. 62, Birkhäuser, 1986, pp. 201-244.

$[\mathrm{DF}] \quad$ S. DeMichelis and M. Freedman, Uncountably many exotic $\mathbb{R}^{4}$ 's in standard 4-space, J. Diff. Geom. 35 (1992), 219-254.

[Do] S. Donaldson, Polynomial invariants for smooth four-manifolds, Topology 29 (1990), 257-315.

[E1] Y. Eliashberg, Classification of overtwisted contact structures on 3-manifolds, Invent. Math. 98 (1989), $623-637$.

[E2] - Topological characterization of Stein manifolds of dimension > 2, Int. J. of Math. 1 (1990), $29-46$.

[E3] - Filling by holomorphic discs and its applications, Geometry of Low-Dimensional Manifolds: 2, Proc. Durham Symp 1989, London Math. Soc. Lecture Notes, vol. 151, Cambridge Univ. Press, 1990, pp. 45-67.

[E4] Contact 3-Manifolds twenty years since J. Martinet's work, Ann. Inst. Fourier 42 (1992), 165-192.

[E5] Legendrian and transversal knots in tight contact 3-manifolds, Topological Methods in Modern Mathematics (1993), 171-193.

[E6] - Symplectic geometry of plurisubharmonic functions, Proceedings, NATO conference on symplectic geometry, Montreal, 1996, to appear.

[EF] Y. Eliashberg and M. Fraser, Classification of topologically trivial Legendrian knots, Proceedings, NATO conference 


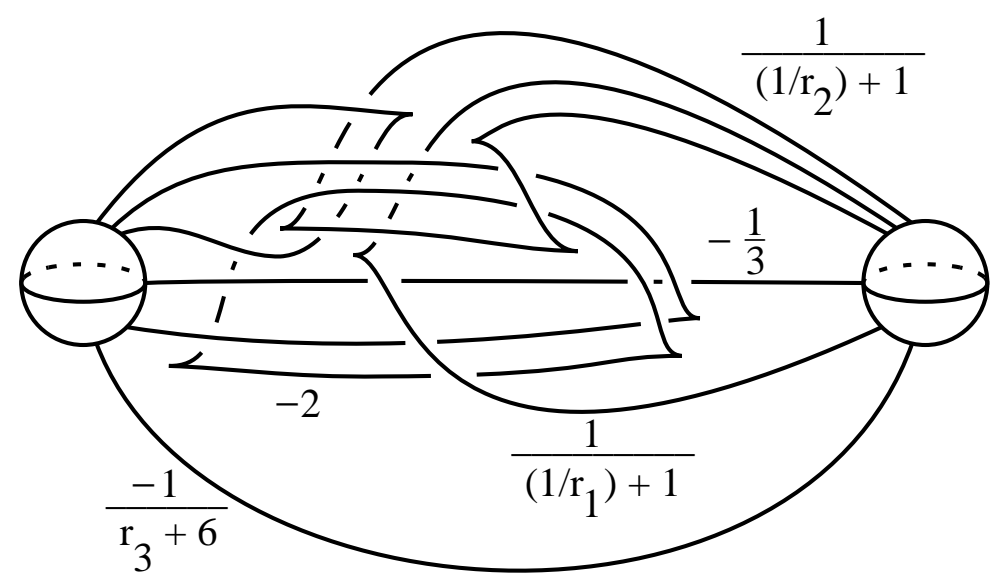

FIGURE 53

on symplectic geometry, Montreal, 1996, to appear.

[EG] Y. Eliashberg and M. Gromov, Convex symplectic manifolds, Proc. Symp. Pure Math. 52, Part 2, 135-162, Amer. Math. Soc., Providence, RI, 1991.

[F] M. Freedman, The topology of four-dimensional manifolds, J. Diff. Geom. 17 (1982), 357-453.

[FQ] M. Freedman and F. Quinn, Topology of 4-Manifolds, Princeton Math. Ser., 39, Princeton Univ. Press, Princeton, NJ, 1990.

[FM] R. Friedman and J. Morgan, Smooth Four-Manifolds and Complex Surfaces, Ergebnisse der Mathematik, 3 Folge, Band 27, Springer, Berlin.

[Gi1] E. Giroux, Convexite en topologie contact, Comm. Math. Helv. 66 (1991), 637-677.

[Gi2] Une structure de contact, meme tendue est plus ou moins tordue, Ann. Scient. Ecole Normale Sup. $\mathbf{2 7}$ (1994), 697-705.

[G1] R. Gompf, Three exotic $\mathbb{R}^{4}$ 's and other anomalies, J. Diff. Geom 18 (1983), 317-328.

[G2] - An infinite set of exotic $\mathbb{R}^{4}$ 's, J. Diff. Geom 21 (1985), 283-300.

[G3] , Spin ${ }^{c}$-structures and homotopy equivalences, MSRI preprint 1997-053.

[GS] R. Gompf and A. Stipsicz, An Introduction to 4-Manifolds and Kirby Calculus, book in preparation.

[Gr] J.W. Gray, Some global properties of contact structures, Ann. Math. 69 (1959), 421-450.

[Gro] M. Gromov, Pseudoholomorphic curves in symplectic manifolds, Invent. Math. 82 (1985), 307-347.

$[\mathrm{HH}] \quad$ F. Hirzebruch and H. Hopf, Felder von Flächenelementen in 4-dimensionalen Mannigfaltigkeiten, Math. Ann. 136 (1958), 156-172.

[Ka] S. Kaplan, Constructing 4-manifolds with given almost framed boundaries, Trans. AMS 254 (1979), $237-263$.

[K] R. Kirby, The topology of 4-manifolds, Lecture Notes in Math., vol. 1374, Springer, 1989.

[KS] R. Kirby and L. Siebenmann, Foundational essays on topological manifolds, smoothings and triangulations, Ann. Math. Studies 88, Princeton Univ. Press, 1977.

[KM] P. Kronheimer and T. Mrowka, Monopoles and contact structures, preprint, 1996.

$[\mathrm{Ku}]$ G. Kuperberg, Non-involutory Hopf algebras and 3-manifold invariants, Duke Math. J. 84 (1996), 83-129.

[LM] P. Lisca and G. Matić, Tight contact structures and Seiberg-Witten invariants, Invent. Math. (to appear).

[M1] J. Milnor, Spin structures on manifolds, L'Ensignement Math 8 (1962), 198-203.

[M2] , Topology from the Differentiable Viewpoint, U. of Virginia Press, 1965.

[NR] W. Neumann and F. Raymond, Seifert manifolds, plumbings, $\mu$-invariant and orientation-reversing maps, Algebraic and Geometric Topology, Lecture Notes in Math, vol. 664, Springer, 1978, pp. 163-196.

[P] L. Pontrjagin, A classification of mappings of the three-dimensional complex into the two dimensional sphere, Matematicheskii Sbornik 9 (series 2) (1941), 331-363.

[Q] F. Quinn, Ends of maps. III: dimensions 4 and 5, J. Diff. Geom. 17 (1982), 503-521.

[Ro] D. Rolfsen, Knots and links, Publish or Perish, Berkeley, 1976.

[R1] L. Rudolph, Quasipositive annuli (constructions of quasipositive knots and links, IV), J. Knot Theory and its Ram. 1 (1992), 451-466.

[R2] - An obstruction to sliceness via contact geometry and "classical" gauge theory, Invent. Math. 119 (1995), $155-163$.

[S] J. Świątkowski, On the isotopy of Legendrian knots, Ann. Glob. Anal. Geom. 10 (1992), 195-207.

[T] C. Taubes, The Seiberg-Witten and Gromov invariants, Math. Res. Letters 2 (1995), 221-238.

[W] A. Weinstein, Contact surgery and symplectic handlebodies, Hokkaido Math. J. 20 (1991), 241-251. 
E-mail address: gompf@math.utexas.edu 